\title{
Modeling Crustal Deformation near Active Faults and Volcanic Centers-A Catalog of Deformation Models
}

Chapter 1 of

Section B, Modeling of Volcanic Processes

Book 13, Volcanic Monitoring

Techniques and Methods 13-B1 



\section{Modeling Crustal Deformation near Active Faults and Volcanic Centers-A Catalog of Deformation Models}

By Maurizio Battaglia, Peter F. Cervelli, and Jessica R. Murray

Chapter 1 of

Section B, Modeling of Volcanic Processes

Book 13, Volcanic Monitoring

Techniques and Methods 13-B1 


\title{
U.S. Department of the Interior SALLY JEWELL, Secretary
}

\section{U.S. Geological Survey Suzette M. Kimball, Acting Director}

\author{
U.S. Geological Survey, Reston, Virginia: 2013
}

For more information on the USGS - the Federal source for science about the Earth, its natural and living resources, natural hazards, and the environment, visit http://www.usgs.gov or call 1-888-ASK-USGS.

For an overview of USGS information products, including maps, imagery, and publications, visit http://www.usgs.gov/pubprod

To order this and other USGS information products, visit http://store.usgs.gov

Any use of trade, firm, or product names is for descriptive purposes only and does not imply endorsement by the U.S. Government.

Although this information product, for the most part, is in the public domain, it also may contain copyrighted materials as noted in the text. Permission to reproduce copyrighted items must be secured from the copyright owner.

Suggested citation:

Battaglia, Maurizio, Cervelli, P.F., and Murray, J.R., 2013, Modeling crustal deformation near active faults and volcanic centers-A catalog of deformation models: U.S. Geological Survey Techniques and Methods, book 13, chap. B1, 96 p., http://pubs.usgs.gov/tm/13/b1. 


\section{Contents}

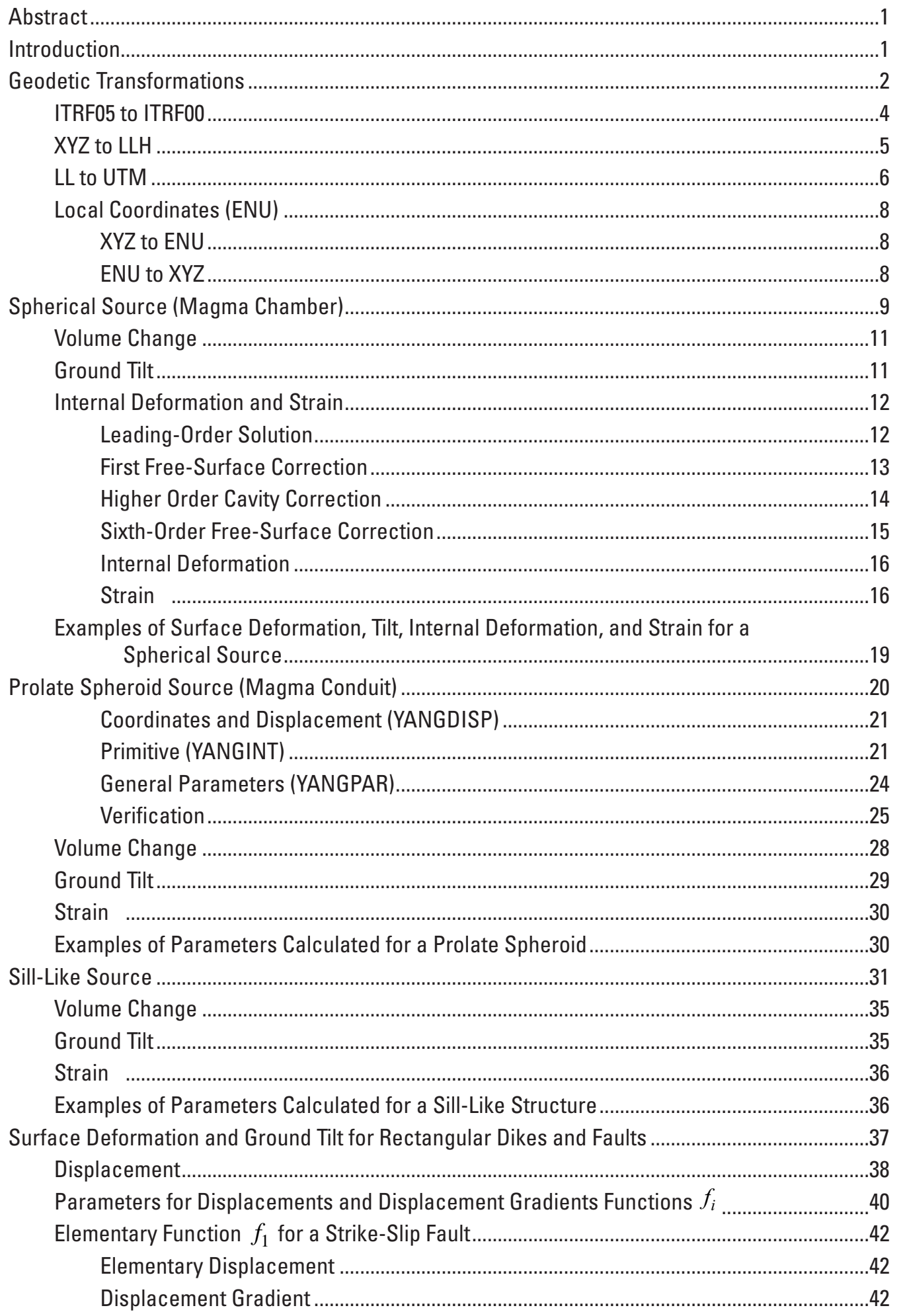




\section{Contents-Continued}

Surface Deformation and Ground Tilt for Rectangular Dikes and Fault-Continued

Elementary Function $f_{2}$ for a Dip-Slip Fault......................................................................42

Elementary Displacement ........................................................................................42

Displacement Gradient .............................................................................................

Elementary Function $f_{3}$ for a Tensile Crack ..................................................................4

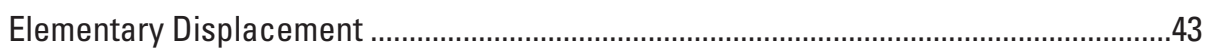

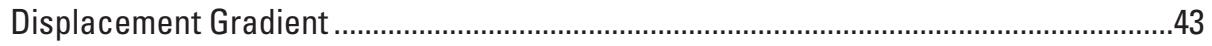

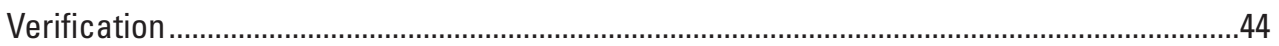

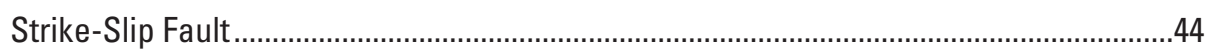

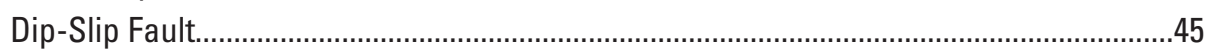

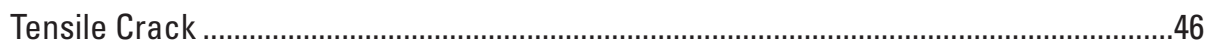

Examples of Surface Deformation and Ground Tilt for Three Types of Faults.........................48

Internal Deformation and Strain for Rectangular Dikes and Faults ...............................................49

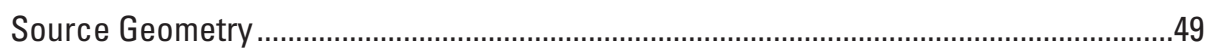

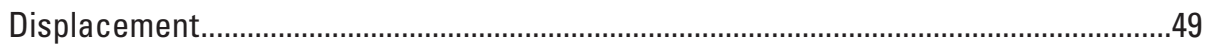

Step 1—Elementary Displacement and Gradient ……..........................................50

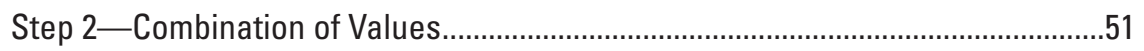

Step 3-Computation of Deformation in Okada's Coordinate System....................51

Step 4-Rotate Deformation Back to Cartesian Coordinate System........................51

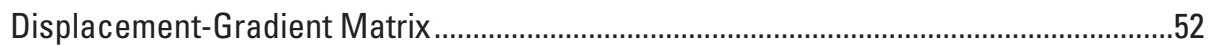

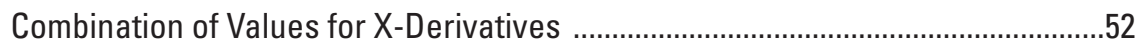

Combination of Values for Y-Derivatives...............................................................52

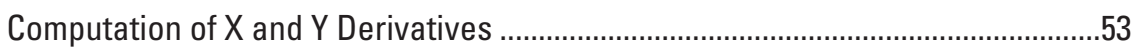

Rotation of the Displacement-Gradient Matrix Back to Cartesian

Coordinate System ………………………………………….......................53

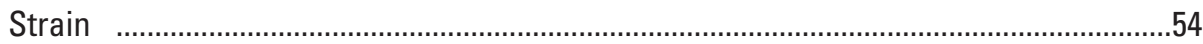

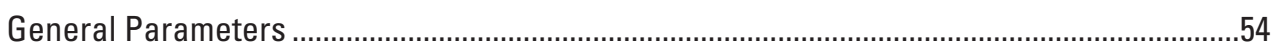

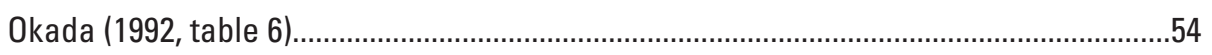

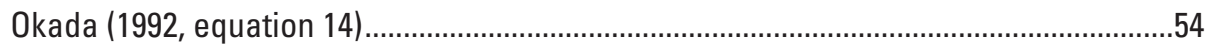

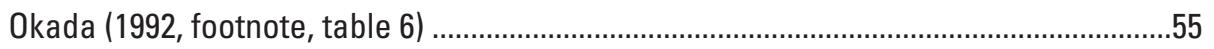

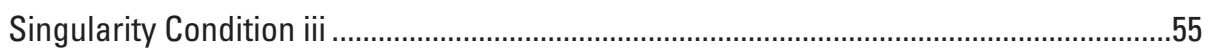

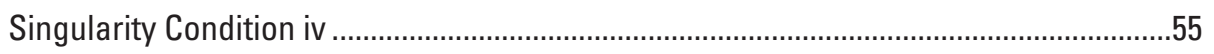

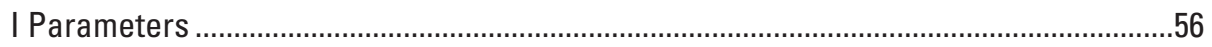

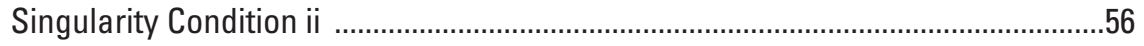

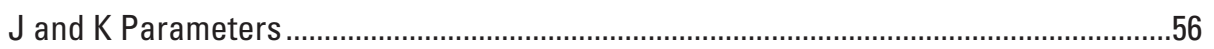

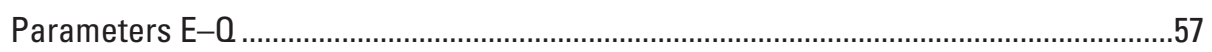

Function $f_{A}$ (Infinite Medium) ...................................................................................58

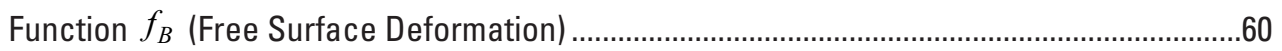

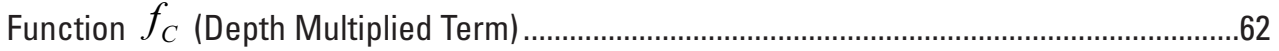

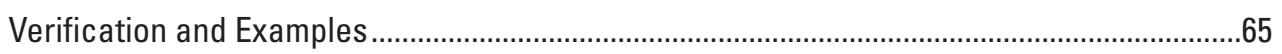

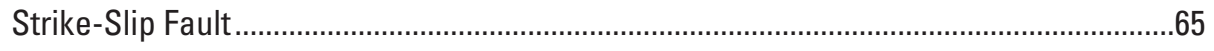

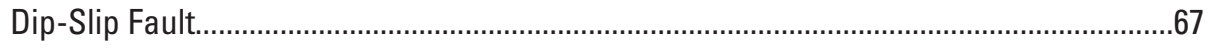

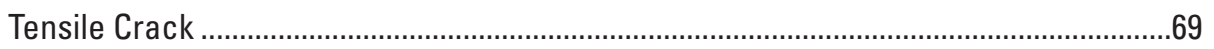




\section{Contents-Continued}

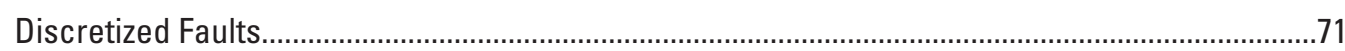

Fault Segments and Subsegments ...................................................................................

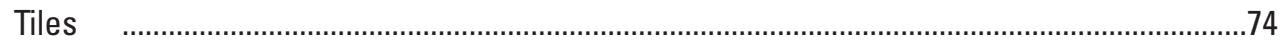

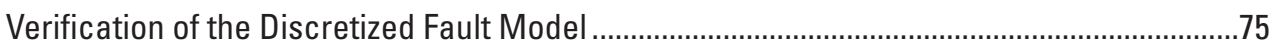

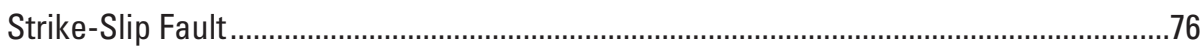

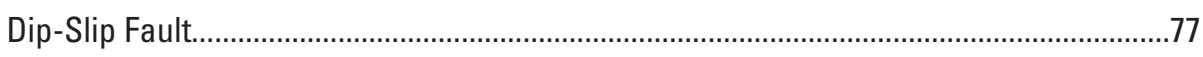

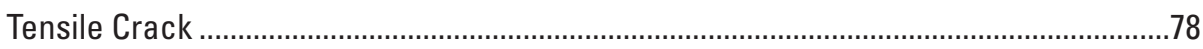

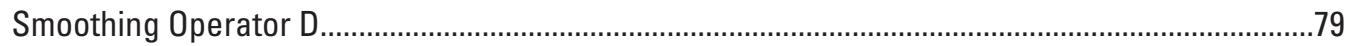

Building the Smoothing Operator..................................................................................

Computing the Location of the Center Point of Each Tile..............................................80

Computing Distances Between the Center Points of Tiles ..............................................81

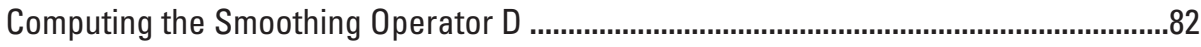

Case 1-Fault does not Break the Surface ..............................................................82

Case 2-Fault Breaks the Surface ...........................................................................83

Case 3-Dike Opening at the Base of the Dislocation............................................83

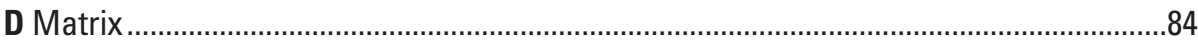

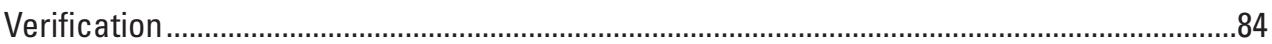

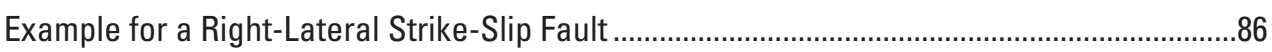

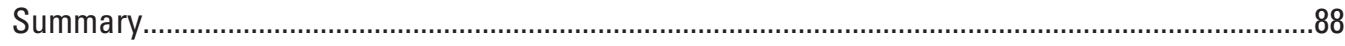

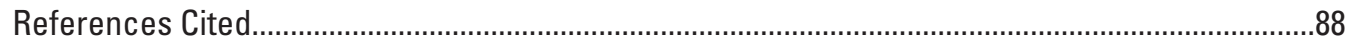

Appendix 1. Mathematical Methods for Computing Displacement .................................................90

\section{Figures}

1. Diagram showing coordinate systems..............................................................................

2. Diagram showing definition of boundary conditions and geometry for a spherical source ...................................................................................................................

3. Graphs showing free-surface deformation caused by a pressurized spherical magma chamber

4. Diagram showing comparison between the dimensionless volume change of a pressurized sphere calculated by use of equation 19 and a finite element method numerical model

5. Diagram showing comparison between calculations of ground tilt in the east and north direction by a two-dimensional analytical model, and a finite element method numerical model

6. Diagram showing definition of dimensionless boundary conditions and coordinates for a spherical source .......................................................................13

7. Graph showing first free-surface correction ..................................................................14

8. Graph showing higher order cavity correction at the free surface ..............................15

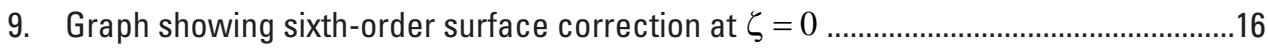

10. Graphs showing internal deformation caused by a spherical source ............................17

11. Graphs showing internal strain caused by a spherical source .......................................18 


\section{Figures}

12. Diagrams showing definition of coordinates system and boundary-value problem for a spheroidal source.

13. Flow chart for coding Yang and others' (1988) expressions for displacement, tilt, and strain

14. Graphs showing free-surface deformation caused by the inflation of a vertical prolate spheroid $\left(\phi=0^{\circ}\right)$ for different values of the geometric aspect ratio $A$.

15. Graphs showing free-surface deformation caused by the inflation of a tilted $\left(\theta\right.$ unequal to $0^{\circ}$ ) and rotated ( $\phi$ unequal to $\left.0^{\circ}\right)$ prolate spheroid

16. Graphs showing internal deformation caused by the inflation of a tilted and rotated prolate spheroid.

17. Graph showing verification of the empirical correction for the volume change of a pressurized prolate spheroid

18. Graphs showing ground tilt caused by the inflation of a tilted and rotated prolate spheroid

19. Graph showing internal strain caused by the inflation of a tilted and rotated prolate spheroid

20. Graph showing definition of boundary conditions and geometry for a sill-like source

21. Graph showing matlab function fialko01.m flow chart for calculating and coding the parameters for a sill-like source

22. Graphs showing surface deformation due to the inflation of a sill-like source; internal deformation.

23. Graph showing volume change for a sill-like source .....................................................35

24. Graphs showing ground tilt caused by the inflation of a sill-like source ..........................35

25. Graphs showing internal strain caused by the inflation of a sill-like source ...................36

26. Program flow chart for rectangular dislocations ...........................................................37

27. Diagrams showing Geometry of the dislocation model in a Cartesian coordinate system; Geometry of the source model

28. Vector and profile plots of the displacement field of a strike-slip fault

29. Vector and profile plots of the displacement field of a dip-slip fault

30. Vector and profile plots of the displacement field of a tensile crack

31. Profile plots showing tilt caused by a tensile crack .....................................................4

32. Program flow chart for rectangular dislocations ........................................................4

33. Vector and profile plots showing displacement fieldand straincaused by a strike-slip fault

34. Graphs showing vector and profile plots of the displacement field and strain caused by a dip-slip fault

35. Graphs showing vector and profile plots of the displacement field and strain caused by a tensile crack

36. Graph showing representation of the fault geometry by segments, subsegments, and tiles.

37. The Matlab script for coding a discretized fault includes four major steps....................72

38. Diagrams showing segment, subsegment, and tile geometry... 


\section{Figures-Continued}

39. Graphs showing fault geometry and vector plot and profile plots of the surface displacement caused by a right-lateral strike-slip fault.

40. Graphs showing fault geometry and vector plot and profile plots of the surface displacement caused by a reverse dip-slip fault

41. Graphs showing fault geometry and vector plot and profile plots of the surface displacement caused by the opening of a tensile crack .................................................78

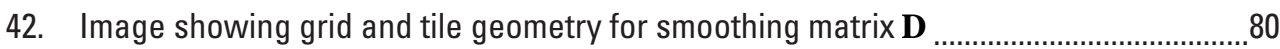

43. Grid used to compute the second-order finite-difference operator $\mathbf{D}$; fault divied into $P=2$ rows, $N=9$ columns, and 18 tiles of different width; smoothing operator $\mathbf{D}$ for the fault in $B$.

44. Graph showing fault geometry used to validate the algorithm for the smoothing operator D

45. Color maps for the smoothing operator, slip distribution, and finite difference

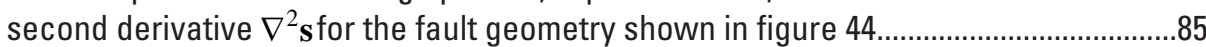

46. Graph showing fault geometry employed in the example ................................................86

\section{Tables}

1. List of earthquake and volcano deformation sources described in this report.................2

2. The 14 transformation parameters between ITRF05 and ITRF00 ......................................4

3. Comparison of IRTF00 and IRTF05 parameters from itrf052itrf00.m with results from the National Geodetic Survey code HTDP for epochs 2000 and 2010 ............................

4. Comparison of Matlab function xyz2llh.m with NGS code HTDP ...................................5

5. Comparison of coordinates for the International Global Survey site FLIN computed in the UTM system in ArcGIS and II2utm.m (datum WGS 84)

6. Conversion of coordinates of GPS sites near the Augustine volcano from IRTF

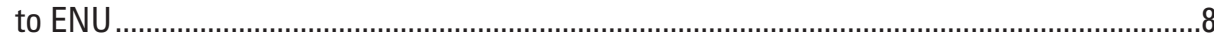

7. Surface deformation, tilt, and internal deformation ..................................................19

8. Corrections for internal deformation .....................................................................19

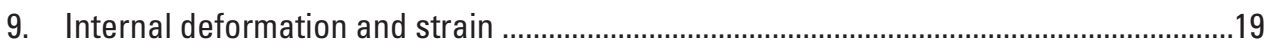

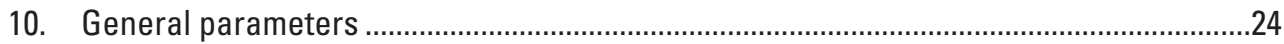

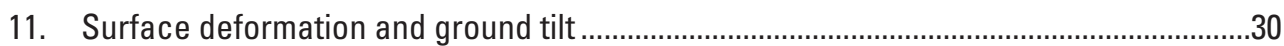

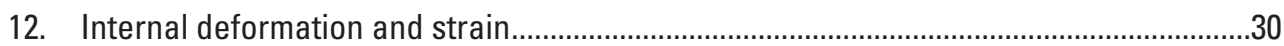

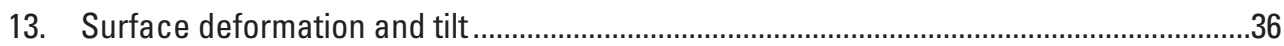

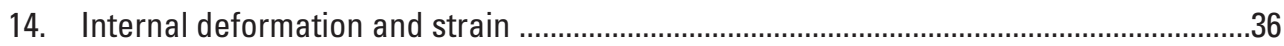

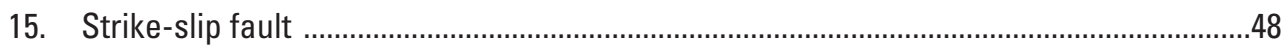

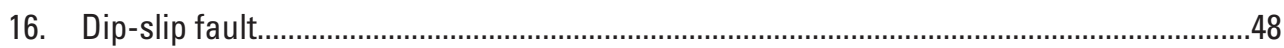

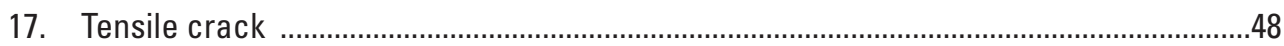

18. Strike-slip fault (internal deformation and strain; function $f_{A}$ ) ...................................58

19. Dip-slip fault (internal deformation and strain; function $f_{A}$ ) ........................................58

20. Tensile crack (internal deformation and strain; function $f_{A}$ ) .......................................59

21. Scaling used to compute the dimensional displacement (function $f_{A}$ ).........................59

22. Strike-slip fault (internal deformation and strain; function $f_{B}$ ) ....................................60

23. Dip-slip fault (internal deformation and strain; function $f_{B}$ ) ........................................60

24. Tensile crack (internal deformation and strain; function $f_{B}$ ) .......................................61

25. Scaling (internal deformation and strain; function $f_{B}$ ) ...............................................61 


\section{Tables-Continued}

26. Strike-slip fault (internal deformation and strain; function $f_{C}$ ) .....................................62

27. Dip-slip fault (internal deformation and strain; function $f_{C}$ ) .......................................63

28. Tensile crack (internal deformation and strain; function $f_{C}$ )........................................64

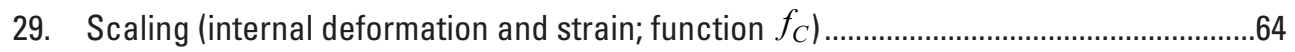

30. Internal deformation and strain around a strike-slip fault.................................................66

31. Internal deformation and strain around a dip-slip fault .............................................68

32. Internal deformation and strain around a tensile crack. ................................................70

33. Parameters for strike-slip fault segments .....................................................................

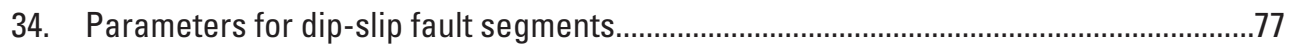

35. Parameters for tensile-crack fault segments ...............................................................78

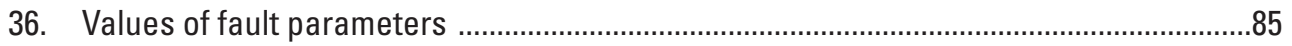

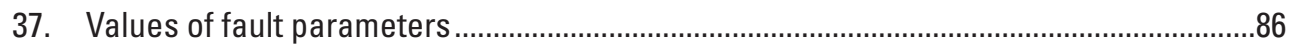

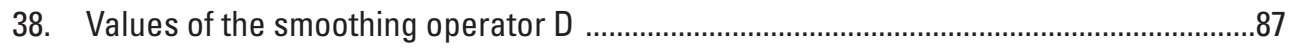

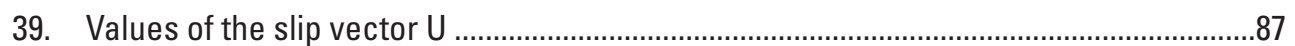

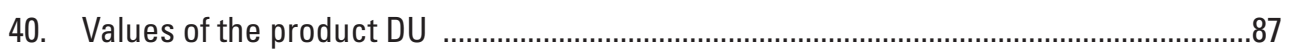

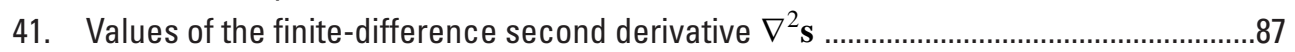

\section{Acronyms}

$\begin{array}{ll}\text { EDM } & \text { Electronic Distance Meter } \\ \text { ENU } & \text { local east-north-up Cartesian coordinate system } \\ \text { FEM } & \text { Finite Element Method } \\ \text { GPS } & \text { Global Positioning System } \\ \text { GTSM } & \text { Gladwin Tensor Strainmeters } \\ \text { InSAR } & \text { Interferometric synthetic aperture radar } \\ \text { ITRF } & \text { International Terrestrial Reference Frame } \\ \text { LLH } & \text { Latitude, longitude, height } \\ \text { LL } & \text { Latitude, longitude } \\ \text { PBO } & \text { Plate Boundary Observatory } \\ \text { UTM } & \text { Universal Transverse Mercator } \\ \text { WGS 84 } & \text { World Geodetic System 1984 }\end{array}$




\title{
Modeling Crustal Deformation near Active Faults and Volcanic Centers-A Catalog of Deformation Models
}

\author{
By Maurizio Battaglia1,2, Peter F. Cervelli1, and Jessica R. Murray ${ }^{1}$
}

\begin{abstract}
This manual provides the physical and mathematical concepts for selected models used to interpret deformation measurements near active faults and volcanic centers. The emphasis is on analytical models of deformation that can be compared with data from the Global Positioning System (GPS) receivers, Interferometric synthetic aperture radar (InSAR), leveling surveys, tiltmeters and strainmeters. Source models include pressurized spherical, ellipsoidal, and horizontal penny-shaped geometries in an elastic, homogeneous, flat half-space. Vertical dikes and faults are described following the mathematical notation for rectangular dislocations in an elastic, homogeneous, flat half-space. All the analytical expressions were verified against numerical models developed by use of COMSOL Multyphics, a Finite Element Analysis software (http://www.comsol.com). In this way, typographical errors present were identified and corrected. Matlab scripts are also provided to facilitate the application of these models.
\end{abstract}

\section{Introduction}

Deformation of the Earth's crust can arise from tectonic and volcanic forces and from human activities such as aquifer withdrawal or geothermal exploitation. Mathematical models of how the crust deforms in response to different physical processes are required to distinguish among possible sources of deformation. These models can also help to characterize deformation-driving processes and to constrain source location, size, orientation, and strength. This information is valuable for hazards forecasting and mitigation, assessment of anthropogenic environmental impact, land-use planning, and other applications. A key assumption behind geodetic monitoring of faults and volcanoes is that ground deformation of the Earth's surface reflects tectonic and volcanic processes at depth (for example, fault slip and mass transport) that are transmitted to the surface through the mechanical properties of the crust (Dzurisin, 2003).

This paper discusses the application of analytical models to fit and interpret ground deformation. Analytical models offer a closed-form description of the source of crustal deformation (table 1). This means that, in principle, it is possible to readily infer the relative importance of any of the source parameters. Although analytical models are based on numerous simplifications (for example, the assumption that the crust is a homogenous, isotropic, elastic, flat half-space) that make the set of differential equations describing the problem tractable, they can take into account a vast array of source geometries. The careful use of analytical models, together with high-quality data sets, can yield valuable insights into the nature of the deformation source.

This report is intended as a practical reference for readers interested in applying mathematical models to investigate volcano and earthquake physics. Under each of the models described, the report first explains the assumptions, applications, and limitations of the model, and then introduces the corresponding analytical formulation. Finally, the report provides tables with numerical examples and Matlab scripts to aid in the use and coding of the formulas.

\footnotetext{
${ }^{1}$ U.S. Geological Survey, 345 Middlefield Road, Menlo Park, California, 94025.

${ }^{2}$ Department of Earth Sciences, Sapienza University of Rome, Italy.
} 
Table 1. List of earthquake and volcano deformation sources described in this report.

\begin{tabular}{lll}
\hline \multicolumn{1}{c}{ Model } & \multicolumn{1}{c}{ Source } & \multicolumn{1}{c}{ Reference } \\
\hline Sphere & Volcano source & \\
Spheroid & Magma chamber & McTigue (1987) \\
Pennyshaped crack & Conduit & Yang and others (1988) \\
Tensile dislocation & Sill & Fialko and others (2001) \\
\hline & Dike & Okada (1985), Okada (1992) \\
\hline Rectangular dislocation & Earthquake source & \\
$\begin{array}{ll}\text { Superposition of rectangular } \\
\text { segments }\end{array}$ & Dip- and strike-slip fault & Okada (1985), Okada (1992) \\
\hline
\end{tabular}

A zipped file with MATLAB scripts can be downloaded from pubs.usgs.gov/tm/13/ b1. The scripts are organized in folders, with each folder corresponding to a chapter in this publication (for example, the folder named "Geodetic transformations" contains MATLAB scripts for the geodetic transformation discuss in the section "Geodetic transformations" of this report). Folders may include subfolders with results from COULOMB 3.3 ("coulomb") and Finite Element Method models ("FEM"), scripts verifying the algorithms ("verification") and MATLAB functions ("functions"). The zipped file includes MATLAB functions for all the sources discussed in this publication and examples of the inversion of Global Positioning System (GPS) data for a spherical, spheroidal and sill-like source (the MATLAB Optimization Toolbox is required to run these examples). Online help can be obtained for each MATLAB function by typing "nameofthefunction help" in the MATLAB command line.

This publication is also designed to allow the user to turn directly to the desired model without reading any other sections (although a general understanding of the concepts and calculus behind the models from other literature will could enhance user experience, see for example Segall, 2010). As a result, some repetitions will be apparent if the manual is read sequentially. Many of the models described in this publication were taken from other credited or standard sources, but the authors themselves derived or rearranged many equations for this publication. All formulas have been checked for possible errors and verified against numerical models (COULOMB 3.3; http://earthquake.usgs.gov/research/modeling/coulomb/; FEM models). Since our emphasis is on describing the characteristics of the models and how they are used, the reader interested in the derivation and calculus of original equations is referred to the original literature. To limit the number of typographical errors, the formulas have been edited directly from the corresponding Matlab functions by using Mathtype.

\section{Geodetic Transformations}

Coordinates can be expressed in systems defined by various datums, all of which are related to each other through geometrical transformations. Geodetic datums describe the size and shape of the Earth, and the origin, orientation, and time derivatives of the coordinate system (fig. 1). The list below includes four common coordinate systems:

1. global Cartesian (XYZ) system - International Terrestrial Reference Frame 2005 (ITRF05; http://itrf.ensg.ign.fr); units are in meters.

2. global geographic system, either latitude, longitude, height (LLH) or latitude, longitude (LL) - International Terrestrial Reference Frame 2000 (ITRF00-WGS 84 ellipsoid; http:// itrf.ensg.ign.fr); units are in degrees for latitude and longitude, and in meters for height.

3. local east-north-up (ENU) Cartesian coordinate system; units are in meters.

4. local Cartesian (XY) system-Universal Transverse Mercator coordinate system (UTMWGS 84 ellipsoid); units are in meters. 
WGS 84 (World Geodetic System), released in 1984 and last revised in 2004 (National Imagery and Mapping Agency (NIMA), 2004) is the standard datum used by the Global Positioning System.

GPS solutions from processing software like GIPSY (https://gipsy-oasis.jpl.nasa.gov) or GAMIT/GLOBK (https://www-gpsg.mit.edu/ simon/gtgk) are usually defined in the ITRF (XYZ) Cartesian coordinate system. GPS cooordinates can also be referenced to a local topocentric datum. The origin of this datum is an arbitrary point on the surface of the Earth (fig. 1). It has three right-handed orthogonal axes: E (for "east") is in the local horizontal plane and points to the geographic east, $\mathrm{N}$ (for "north") is in the local horizontal plane and points to the geographic north, $\mathrm{U}$ (for "up") is vertical (in other words, perpendicular to the local equipotential surface) and points upwards; units are meters. UTM is a local Cartesian (XY) coordinate system based on the Universal Transverse Mercator projection and defined for the whole Earth (U.S. Army, 1987); units are meters. Modeling deformation velocities from GPS requires the transformation of the original ITRF coordinates into local Cartesian coordinates.

Coordinates in ITRF05 can be transformed into ITRF00 by means of a 14-parameter Helmert transformation. ITRF00 and WGS 84 (G1150), the latest realization of the WGS 84 coordinate system, are the same within one cm (NIMA, 2000 and 2004). The OpenGIS Web Map Service (www.opengeospatial.org/standards/wms) offers georeferenced maps in UTM (WGS84). The report provides formulas and Matlab functions to transform coordinates from

1. ITRF05 to ITRF00,

2. ITRF (XYZ) to LLH,

3. LL to UTM (XY), and

4. ITRF (XYZ) to the east-north-up coordinate system (ENU).

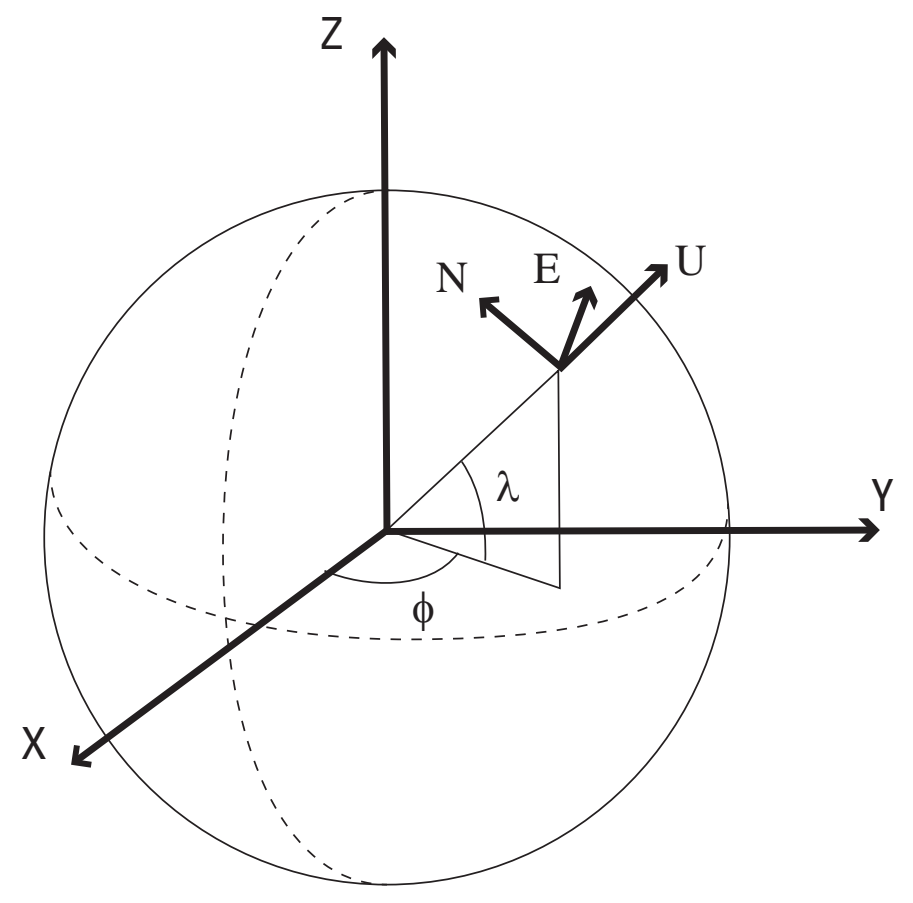

Figure 1. Coordinate systems. XYZ, global Cartesian coordinate system; ENU, local Cartesian coordinate system; $(\lambda, \phi)$ global geographic coordinate system (latitude, longitude). 


\section{ITRF05 to ITRF00}

The 14-parameter Helmert transformation is (Dawson and Woods, 2010)

$$
\left[\begin{array}{c}
X_{00} \\
Y_{00} \\
Z_{00}
\end{array}\right]=\left[\begin{array}{c}
d_{x}+d r_{x}\left(t-t_{0}\right) \\
d_{y}+d r_{y}\left(t-t_{0}\right) \\
d_{z}+d r_{z}\left(t-t_{0}\right)
\end{array}\right]+\left[1+s c+s r c\left(t-t_{0}\right)\right] \mathbf{R}\left[\begin{array}{c}
X_{05} \\
Y_{05} \\
Z_{05}
\end{array}\right]
$$

where $\left[\begin{array}{lll}d_{x} & d_{y} & d_{z}\end{array}\right]$ is the origin shift in $\mathrm{m},\left[\begin{array}{lll}d r_{x} & d r_{y} & d r_{z}\end{array}\right]$ is the shift rate in $\mathrm{m} / \mathrm{yr}, t$ is the time (epoch) in years, $t_{0}=2000.0, s c$ is the dimensionless scaling in ppb and $s r c$ the scaling rate in ppb/yr (table 2).

The rotation matrix $\mathbf{R}$ is the sum of steady $\left(\mathbf{R}_{\mathbf{s}}\right)$ and time dependent $\left(\mathbf{R}_{\mathbf{t}}\right)$ components (see table 3 for a comparison between itr052itrf00.m and standard NGS software)

$$
\mathbf{R}_{\mathbf{s}}=\left[\begin{array}{ccc}
1 & R_{z} & -R_{y} \\
-R_{z} & 1 & R_{x} \\
R_{y} & -R_{x} & 1
\end{array}\right] \text { and } \quad \mathbf{R}_{\mathbf{t}}=\left[\begin{array}{ccc}
0 & R r_{z}\left(t-t_{0}\right) & -\operatorname{Rr}_{y}\left(t-t_{0}\right) \\
-\operatorname{Rr}_{z}\left(t-t_{0}\right) & 0 & R r_{x}\left(t-t_{0}\right) \\
R r_{y}\left(t-t_{0}\right) & -\operatorname{Rr}_{x}\left(t-t_{0}\right) & 0
\end{array}\right]
$$

Table 2. The 14 transformation parameters between ITRF05 and ITRF00.

[See http://itrf.ensg.ign.fr/ITRF_solutions/2005/tp_05-00.php; ITRF, International Terrestrial Reference Frame; $d_{\mathrm{x}}, d_{\mathrm{y}}$, and $d_{\mathrm{z}}$, Cartesian parameters measured in meters; $R_{\mathrm{x}}, R_{\mathrm{y}}$, and $R_{\mathrm{z}}$, angular coordinates; mas, milliarcseconds; $s c$, scaling; ppb, part per billion; $d r_{\mathrm{x}}, d r_{\mathrm{y}}$, and $d r_{\mathrm{z}}$, Cartesian shift rates; m/yr, meters per year;

\begin{tabular}{|c|c|c|c|c|c|c|}
\hline$d_{x}$ (meters) & $d_{y}$ (meters) & $d_{z}$ (meters) & $R_{x}$ (mas) & $R_{y}$ (mas) & $R_{z}$ (mas) & $S c(p p b)$ \\
\hline 0.0001 & -0.0008 & -0.0058 & 0.000 & 0.000 & 0.000 & 0.40 \\
\hline$d r_{x}(\mathrm{~m} / \mathrm{yr})$ & $d r_{y}(\mathrm{~m} / \mathrm{yr})$ & $d r_{z}(\mathrm{~m} / \mathrm{yr})$ & $R r_{x}$ (mas/yr) & $R r_{y}$ (mas/yr) & $R r_{z}$ (mas/yr) & $\operatorname{Src}(\mathrm{ppb} / \mathrm{yr})$ \\
\hline
\end{tabular}
$R r_{\mathrm{x}}, R r_{\mathrm{y}}$, and $R r_{\mathrm{z}}$, angular shift rates; mas/yr, milliarcseconds per year; src, scaling rate; ppb/yr; part per billion per year].

Table 3. Comparison of IRTF00 and IRTF05 parameters from itrf052itrf00.m with results from the National Geodetic Survey code HTDP for epochs 2000 and 2010.

[The difference $\Delta$ between the coordinates is less than 1 millimeter. See http://www.ngs.noaa.gov/TOOLS/Htdp/Htdp. shtml; ITRF, International Terrestrial Reference Frame; HTDP, horizontal time-dependent positioning; coordinates for

\begin{tabular}{|c|c|c|c|}
\hline \multicolumn{4}{|c|}{ Epoch 2000.01.01 } \\
\hline & $\begin{array}{c}X \\
\text { (meters) }\end{array}$ & $\begin{array}{c}\mathrm{Y} \\
\text { (meters) }\end{array}$ & $\begin{array}{c}\mathrm{Z} \\
\text { (meters) }\end{array}$ \\
\hline ITRF05 & -766174.473 & -3611375.309 & 5184056.243 \\
\hline ITRF00 (HDTP) & -766174.473 & -3611375.311 & 5184056.239 \\
\hline ITRF00 & -766174.473 & -3611375.311 & 5184056.239 \\
\hline$\Delta$ & -0.0002 & -0.0002 & 0.0003 \\
\hline \multicolumn{4}{|c|}{ Epoch 2010.05.14 } \\
\hline & $\begin{array}{c}\mathrm{X} \\
\text { (meters) }\end{array}$ & $\begin{array}{c}\mathbf{Y} \\
\text { (meters) }\end{array}$ & $\begin{array}{c}\mathrm{Z} \\
\text { (meters) }\end{array}$ \\
\hline ITRF05 & -766174.661 & -3611375.333 & 5184056.209 \\
\hline ITRF00 (HDTP) & -766174.664 & -3611375.337 & 5184056.191 \\
\hline ITRF00 & -766174.664 & -3611375.337 & 5184056.191 \\
\hline$\Delta$ & 0.0001 & -0.0002 & -0.0001 \\
\hline
\end{tabular}
both epochs are for site FLIN.; $\Delta$, difference] 


\section{XYZ to LLH}

The formulas implemented in xyz21lh.m compute longitude, latitude (both in decimal degrees), and height (in meters) from the ITRF00 cartestian coordinates XYZ. Geodetic positioning LLH is in the WGS 84 (G1150) reference frame. Horizontal datum is referred to WGS 84, and the vertical datum to the WGS 84 ellipsoid. Sign conventions are positive for E and longitude; positive for $\mathrm{N}$ and latitude. The constants for the WGS 84 ellipsoid are (NIMA, 2000, paragraph 3.2)

$$
\begin{array}{ll}
a=6378137 & \text { semimajor axis, in meters, and } \\
f=1 / 298.257223563 & \text { flattening. }
\end{array}
$$

The geometrical parameters of the ellipsoidal coordinate system (Hoffmann-Wellenhof and others, 1997, equations 10.11-10.13) are

$$
\begin{aligned}
b & =(1-f) a & & \text { semi-minor axis, in meters } \\
e^{2} & =2 f-f^{2} & & \text { eccentricity squared } \\
e_{2 \phi} & =\left(a^{2}-b^{2}\right) / b^{2} & & \text { second numerical eccentricity } \\
p & =\sqrt{X^{2}+Y^{2}} & & \text { radius of a parallel }
\end{aligned}
$$

The longitude $(\phi)$, latitude $(\lambda)$, and height $(H)$ are given by

$$
\begin{gathered}
\phi=-\tan ^{-1}\left(\frac{X}{Y}\right) \\
\lambda=\tan ^{-1}\left(\frac{Z+e_{2 \phi} b \sin ^{3} \theta}{p-e^{2} a \cos ^{3} \theta}\right), \quad \theta=\tan ^{-1}\left(\frac{Z a}{p b}\right), \\
H=\frac{p}{\cos \lambda}-N, \quad \text { and } \quad N=\frac{a}{\sqrt{1-e^{2} \sin ^{2} \lambda}},
\end{gathered}
$$

where

$N$ is the radius of curvature.

Finally, longitude and latitude are converted to degrees and the sign is changed so that $\mathrm{E}$ longitude is positive (table 4).

Table 4. Comparison of Matlab function xyz2llh.m with NGS code HTDP.

[See http://www.ngs.noaa.gov/TOOLS/Htdp/Htdp.shtml. Values are for the International Global Navigation Satellite System Survey site FLIN. ITRF, International Terrestrial Reference Frame; WGS, World Geodetic System; HDTP, horizontal time-dependent positioning; DD, decimal degrees; X, Y, and Z, Cartesian coordinates; Long, longitude; Lat, latitude; $\Delta$, difference]

\begin{tabular}{lccc}
\hline & $\mathbf{X}$ (meters) & $\mathbf{Y}$ (meters) & $\mathbf{Z}$ (meters) \\
\hline ITRF05 & -766174.473 & -3611375.309 & 5184056.243 \\
\hline Long (DD) & Lat (DD) & Height (meters) \\
\hline WGS 84 (HDTP) & -101.978033 & 54.725584 & 311.5070 \\
WGS 84 & -101.978033 & 54.725584 & 311.5068 \\
$\Delta$ & -0.000000 & -0.000000 & -0.0002 \\
\hline
\end{tabular}




\section{LL to UTM}

The Matlab function ll2utm.m computes the E (east, meters) and N (north, meters) coordinates on the UTM grid from the WGS 84 (G1150) longitude $(\phi)$ and latitude $(\lambda)$. According to the Defense Mapping Agency (1989) report, "The computations $(* * *)$ are accurate to the nearest 0.001 arc second for geographic coordinates and to the nearest 0.01 meter for grid coordinates", where 0.001 arc second $=3 \times 10^{-7}$ decimal degrees $=4.848 \times 10^{-9}$ rad. The UTM horizontal datum is the WGS 84 ellipsoid, with sign convention positive for E longitude and positive for $\mathrm{N}$ latitude.

The first step is to set the constants for the WGS 84 ellipsoid (NIMA, 2000, paragraph 3.2):

$$
\begin{array}{ll}
a=6378137 & \text { semimajor axis, in meters, and } \\
f=1 / 298.257223563 & \text { flattening. }
\end{array}
$$

and the WGS 84 ellipsoid parameters (Defense Mapping Agency, 1989, paragraph 2-2.1):

$$
\begin{aligned}
& b=(1-f) a \quad \text { semiminor axis, in meters } \\
& e^{2}=2 f-f^{2} \quad \text { eccentricity squared } \\
& e_{2 \phi}=\left(a^{2}-b^{2}\right) / b^{2} \quad \text { second numerical eccentricity } \\
& v=\frac{a}{\sqrt{1-e^{2} \sin ^{2} \phi}} \quad \text { radius of curvature in the prime vertical } \\
& n=\frac{f}{2-f} \\
& A_{\phi}=a\left[1-n+5\left(n^{2}-n^{3}\right) / 4+81\left(n^{4}-n^{5}\right) / 64\right] \\
& B_{\phi}=(3 / 2) a\left[n-n^{2}+7\left(n^{3}-n^{4}\right) / 8+55 n^{5} / 64\right] \\
& C_{\phi}=(15 / 16) a\left[n^{2}-n^{3}+0.75\left(n^{4}-n^{5}\right)\right] \\
& D_{\phi}=(35 / 48) a\left(n^{3}-n^{4}+11 n^{5} / 16\right) \\
& E_{\phi}=(315 / 512) a\left(n^{4}-n^{5}\right) \\
& S=A_{\phi} \phi-B_{\phi} \sin (2 \phi)+C_{\phi} \sin (4 \phi)-D_{\phi} \sin (6 \phi)+E_{\phi} \sin (8 \phi)
\end{aligned}
$$

where $S$ is the meridional arc.

The UTM projection parameters (Defense Mapping Agency, 1989, paragraph 2-2.2) are the longitude measured with respect to the central meridian $\left(\lambda_{0}\right)$, the central scale factor $k_{0}$, false easting (FE), and false northing $(F N)$ :

$$
\begin{array}{ccc}
\Delta \lambda=\lambda-\lambda_{0}, & k_{0}=0.9996, \\
F N=0 \text { or } 10,000,000 & F E=500,000
\end{array}
$$

where $F N$ is 0 for the northern hemisphere and 10,000,000 for the southern hemisphere. 
The following terms are used to calculate the general equations which follow in this section (Defense Mapping Agency, 1989, paragraph 2-2.3):

$$
\begin{aligned}
T_{1}= & S k_{0} \\
T_{2}= & v \sin \phi \cos \phi k_{0} / 2 \\
T_{3}= & \left(T_{2} \cos ^{2} \phi / 12\right)\left(5-\tan ^{2} \phi+9 e_{2 \phi} \cos ^{2} \phi+4 e_{2 p}^{2} \cos ^{4} \phi\right) \\
T_{4}= & \left(T_{2} \cos ^{4} \phi / 360\right)\left(61-58 \tan ^{2} \phi+\tan ^{4} \phi+270 e_{2 \phi} \cos ^{2} \phi-330 \tan ^{2} \phi e_{2 p} \cos ^{2} \phi\right. \\
& +445 e_{2 \phi}^{2} \cos ^{4} \phi+324 e_{2 \phi}^{3} \cos ^{6} \phi-680 \tan ^{2} \phi e_{2 \phi}^{2} \cos ^{4} \phi+ \\
& \left.+88 e_{2 \phi}^{4} \cos ^{8} \phi-600 \tan ^{2} \phi e_{2 \phi}^{3} \cos ^{6} \phi-192 \tan ^{2} \phi e_{2 \phi}^{4} \cos ^{8} \phi\right) \\
T_{5}= & \left(T_{2} \cos ^{6} \phi / 20160\right)\left(1385-3111 \tan ^{2} \phi+543 \tan ^{4} \phi-\tan ^{6} \phi\right) \\
T_{6}= & v \cos \phi k_{0} \\
T_{7}= & \left(T_{6} \cos ^{2} \phi / 6\right)\left(1-\tan ^{2} \phi+e_{2 \phi} \cos ^{2} \phi\right) \\
T_{8}= & \left(T_{6} \cos ^{4} \phi / 120\right)\left(5-18 \tan ^{2} \phi+\tan ^{4} \phi+14 e_{2 \phi} \cos ^{2} \phi-58 \tan ^{2} \phi e_{2 \phi} \cos ^{2} \phi+\right. \\
& \left.+13 e_{2 \phi}^{2} \cos ^{4} \phi+4 e_{2 \phi}^{3} \cos ^{6} \phi-64 \tan ^{2} \phi e_{2 \phi}^{2} \cos ^{4} \phi-24 \tan ^{2} \phi e_{2 \phi}^{3} \cos ^{6} \phi\right) \\
T_{9}= & \left(T_{6} \cos ^{6} \phi / 5040\right)\left(61-479 \tan ^{2} \phi+179 \tan ^{4} \phi-\tan ^{6} \phi\right)
\end{aligned}
$$

The general formulas for the conversion of geographic coordinates to north $(N)$ and east (E) grid coordinates (Defense Mapping Agency, 1989, paragraph 2-5; table 5) are

$$
\begin{aligned}
N & =F N+T_{1}+T_{2} \Delta \lambda^{2}+T_{3} \Delta \lambda^{4}+T_{4} \Delta \lambda^{6}+T_{5} \Delta \lambda^{8} \\
E & =F E+T_{6} \Delta \lambda+T_{7} \Delta \lambda^{3}+T_{8} \Delta \lambda^{5}+T_{9} \Delta \lambda^{7}
\end{aligned}
$$

Table 5. Comparison of coordinates for the International Global Survey site FLIN computed in the UTM system in ArcGIS and II2utm.m (datum WGS 84).

[WGS, World Geodetic System; UTM, Universal Transverse Mercator; GIS, geographic information system; DD, decimal degrees; X, Y, and Z, global Cartesian coordinates; Long, longitude; Lat, latitude; $\Delta$, difference]

\begin{tabular}{lcc}
\hline & Long (DD) & Lat (DD) \\
\hline WGS 84 (G1150) & -101.9780327 & 54.7255844 \\
\hline UTM (ArcGIS) & $\mathbf{E}$ (meters) & $\mathbf{N}$ (meters) \\
UTM & 308230.414 & 6068325.668 \\
$\Delta$ & 308230.413 & 6068325.666 \\
\hline
\end{tabular}




\section{Local Coordinates (ENU)}

\section{$\mathrm{XYZ}$ to ENU}

The Matlab function xyz2enu.m transforms global Cartestian coordinates (XYZ) into local east-north-up (ENU) Cartesian coordinates. The origin of the local coordinate system is set to the minimum value of the (XYZ) coordinates. The transformation comprises two steps:

1. Transform the origin from XYZ coordinates $\left(X_{0}, Y_{0}, Z_{0}\right)$ to LL coordinates $\left(\phi_{0}, \lambda_{0}\right)$, and

2. Convert XYZ coordinates to ENU (Hoffmann-Wellenhof and others, 1997, p. 149) through the equation

$$
\left[\begin{array}{l}
E \\
N \\
U
\end{array}\right]=\left[\begin{array}{ccc}
-\sin \lambda_{0} & \cos \lambda_{0} & 0 \\
-\sin \phi_{0} \cos \lambda_{0} & -\sin \phi_{0} \sin \lambda_{0} & \cos \phi_{0} \\
\cos \phi_{0} \cos \lambda_{0} & \cos \phi_{0} \sin \lambda_{0} & \sin \phi_{0}
\end{array}\right]\left[\begin{array}{c}
X-X_{0} \\
Y-Y_{0} \\
Z-Z_{0}
\end{array}\right]
$$

\section{ENU to $X Y Z$}

This function (enu2xyz.m) implements the inversion of the XYZ to ENU transformation. A local reference point (for example, the origin) in $\mathrm{XYZ}$ is used to tie the coordinates to ITRF (see example in table 6 for the Augustine volcano in Alaska):

$$
\left[\begin{array}{c}
X \\
Y \\
Z
\end{array}\right]=\left[\begin{array}{ccc}
-\sin \lambda_{0} & -\sin \phi_{0} \cos \lambda_{0} & \cos \phi_{0} \cos \lambda_{0} \\
\cos \lambda_{0} & -\sin \phi_{0} \sin \lambda_{0} & \cos \phi_{0} \sin \lambda_{0} \\
0 & \cos \phi_{0} & \sin \phi_{0}
\end{array}\right]\left[\begin{array}{c}
E \\
N \\
U
\end{array}\right]+\left[\begin{array}{c}
X_{0} \\
Y_{0} \\
Z_{0}
\end{array}\right] .
$$

Table 6. Conversion of coordinates of GPS sites near the Augustine volcano from IRTF to ENU.

[See Cervelli and others (2006) for original coordinates; ITRF, International Terrestrial Reference Frame; ENU, east north up (local coordinates); $\left(\lambda_{0}, \phi_{0}\right)$ are the latitude and longitude of the local origin (Augustine volcano); $\left(\mathrm{X}_{0}, \mathrm{Y}_{0}, \mathrm{Z}_{0}\right)$ are the ITRF Cartesian

\begin{tabular}{|c|c|c|c|}
\hline & \multicolumn{3}{|c|}{ ITRF coordinates } \\
\hline & $\begin{array}{c}\mathrm{X} \\
\text { (meters) }\end{array}$ & $\begin{array}{c}\mathrm{Y} \\
\text { (meters) }\end{array}$ & $\begin{array}{c}\mathrm{Z} \\
\text { (meters) }\end{array}$ \\
\hline AC59 & -2900773.239 & -1440890.357 & 5476476.756 \\
\hline AUGL & -2911728.045 & -1461015.567 & 5465156.878 \\
\hline AV01 & -2915632.507 & -1456172.186 & 5464818.582 \\
\hline AV02 & -2916881.537 & -1458858.111 & 5463145.488 \\
\hline AV03 & -2913037.130 & -1456338.750 & 5466001.512 \\
\hline AV04 & -2915070.452 & -1456916.823 & 5465417.529 \\
\hline \multirow[t]{3}{*}{ AV05 } & -2914535.420 & -1458049.359 & 5465541.274 \\
\hline & \multicolumn{3}{|c|}{ ENU coordinates } \\
\hline & $\begin{array}{c}\text { E } \\
\text { (meters) }\end{array}$ & $\underset{\text { (meters) }}{\mathbf{N}}$ & $\underset{\text { (meters) }}{\mathbf{U}}$ \\
\hline AC59 & 2606.223 & 9556.053 & 8918.654 \\
\hline AUGL & 15009.630 & -5668.214 & -595.141 \\
\hline AV01 & -7450.800 & 240.078 & 2191.218 \\
\hline AV02 & -3437.328 & 6941.531 & -1299.731 \\
\hline AV03 & 2404.740 & 1320.257 & -752.401 \\
\hline AV04 & 679.336 & -816.519 & 4293.685 \\
\hline AV05 & -16943.480 & 11095.571 & 238.721 \\
\hline
\end{tabular}
coordinates of the local origin (Augustine volcano). Footnotes 1 and 2 are the numerical values of $\left(\lambda_{0}, \phi_{0}\right)$ and $\left.\left(\mathrm{X}_{0}, \mathrm{Y}_{0}, \mathrm{Z}_{0}\right)\right]$ 


\section{Spherical Source (Magma Chamber)}

The deformation due to an expanding or contracting magma chamber has frequently been modeled by a dilatation source in an elastic half space. The most commonly used is the Mogi, or point, source (Masterlark, 2007). The model simulates a small spherical source embedded in a homogeneous, isotropic, elastic, flat half-space (fig. 2).

The appeal of Mogi's model lies in its combination of computational simplicity and remarkable ability to predict radially symmetric deformation caused by magma intrusion. The accuracy of an interpretation based on Mogi's model, however, is subject to the suitability of the initial assumptions - an often overlooked consideration (Masterlark, 2007). For example, Mogi's point-source model can explain stresses and displacements far away from the magma chamber, but the stresses are infinite at the source. In contrast, McTigue's (1987) formulation provides an analytical solution with higher order terms that account for the finite shape of a spherical body. Thus, the local stresses at, and far from, the boundary of a chamber remain finite and can be calculated. McTigue's results (1987) for vertical $\left(u_{z}\right)$ and radial $\left(u_{r}\right)$ surface deformation caused by a pressurized ( $\Delta P$ ) spherical magma chamber of radius $a$ and at position $\left(x_{0}, y_{0}, z_{0}\right)$ (fig. 2$)$ can be written in the form

$$
u_{z}=(1-v) \frac{\Delta P a^{3}}{\mu} \frac{z_{0}}{\left(r^{2}+z_{0}^{2}\right)^{3 / 2}}\left\{1-\left(\frac{a}{z_{0}}\right)^{3}\left[\frac{1+v}{2(7-5 v)}-\frac{15(2-v)}{4(7-5 v)} \frac{z_{0}^{2}}{r^{2}+z_{0}^{2}}\right]\right\},
$$

and

$$
u_{r}=(1-v) \frac{\Delta P a^{3}}{\mu} \frac{r}{\left(r^{2}+z_{0}^{2}\right)^{3 / 2}}\left\{1-\left(\frac{a}{z_{0}}\right)^{3}\left[\frac{1+v}{2(7-5 v)}-\frac{15(2-v)}{4(7-5 v)} \frac{z_{0}^{2}}{r^{2}+z_{0}^{2}}\right]\right\}
$$

where $z_{0}$ is the depth of the source (a positive number), $v$ the Poisson's ratio, and $\mu$ the shear modulus. A direct consequence of the assumption of a point source of dilatation is that the magma-chamber radius $a$ and pressure change $\Delta P$ are inseparable because $\Delta P a^{3}$ is the strength of the point singularity. This is why point source models typically calculate volume rather than pressure changes.

When the radius $a$ is small relative to the depth $z_{0}$, so that $\left(a / z_{0}\right)^{3}$ is much less than 1, the Mogi assumptions are reasonable, and the McTigue (1987) correction is not needed. For example, when $a=z_{0} / 2$, the correction is only 12 percent, and it decreases to 3 percent for $a=z_{0} / 3$. This means that, if the overall accuracy of the geodetic survey is within 3-12 percent, the Mogi's model is sufficient to fit the deformation. If the site effects induced by shallow heterogeneities and topography account for as little as 3-12 percent of the deformation signal, the Mogi's model is again a good approximation.

We can use equations 16 and 17 and to compute the three components of surface deformation (for example, the components measured by GPS receivers) aligned to geographic east (x-axis), north (y-axis) and up (z-axis), as shown in figure 3 (see table 7 at the end of chapter for numerical examples).

$$
\left[\begin{array}{lll}
u_{x} & u_{y} & u_{z}
\end{array}\right]=\left[\begin{array}{lll}
u_{r} \frac{x}{r} & u_{r} \frac{y}{r} & u_{z}
\end{array}\right]
$$


$\boldsymbol{A}$

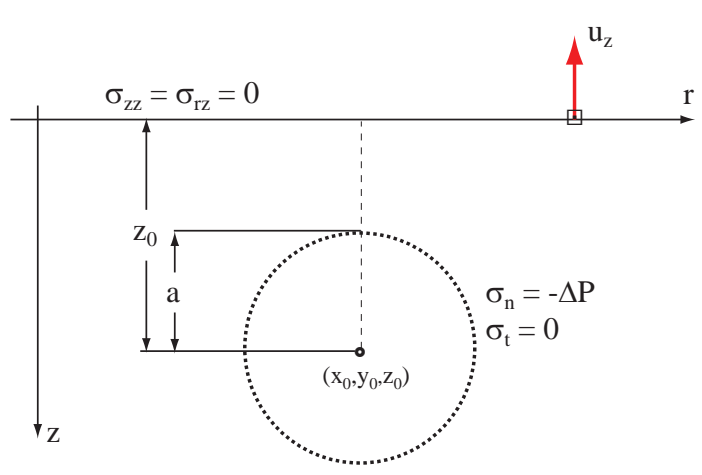

B

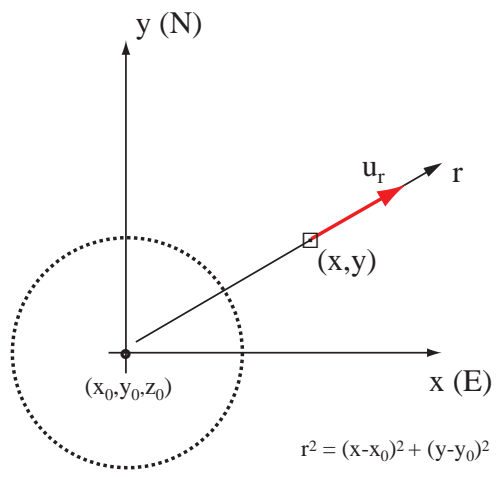

Figure 2. Definition of boundary conditions and geometry for a spherical source (modified from McTigue, 1987). A, Vertical cross section showing he boundary conditions: the normal stresses $\sigma_{r z}$ and $\sigma_{z z}$ are equal to zero at the free surface, whereas the normal stress $\sigma_{n}$ is equal to the pressure change at the surface of the spherical magma chamber, and the tangential stress $\sigma_{t}$ is zero. $r$ and $z$ are local cylindrical coordinates, $a$ is the radius of the spherical source, $\left(x_{0}, y_{0}, z_{0}\right)$ is the source location, $\mathrm{u}_{\mathrm{z}}$ is the uplift. $B$, The local coordinate system at the earth's surface. $\mathrm{x}$ (east) and $\mathrm{y}$ (north) are local Cartesian coordinates, $\mathrm{u}_{\mathrm{r}}$ is the radial displacement.
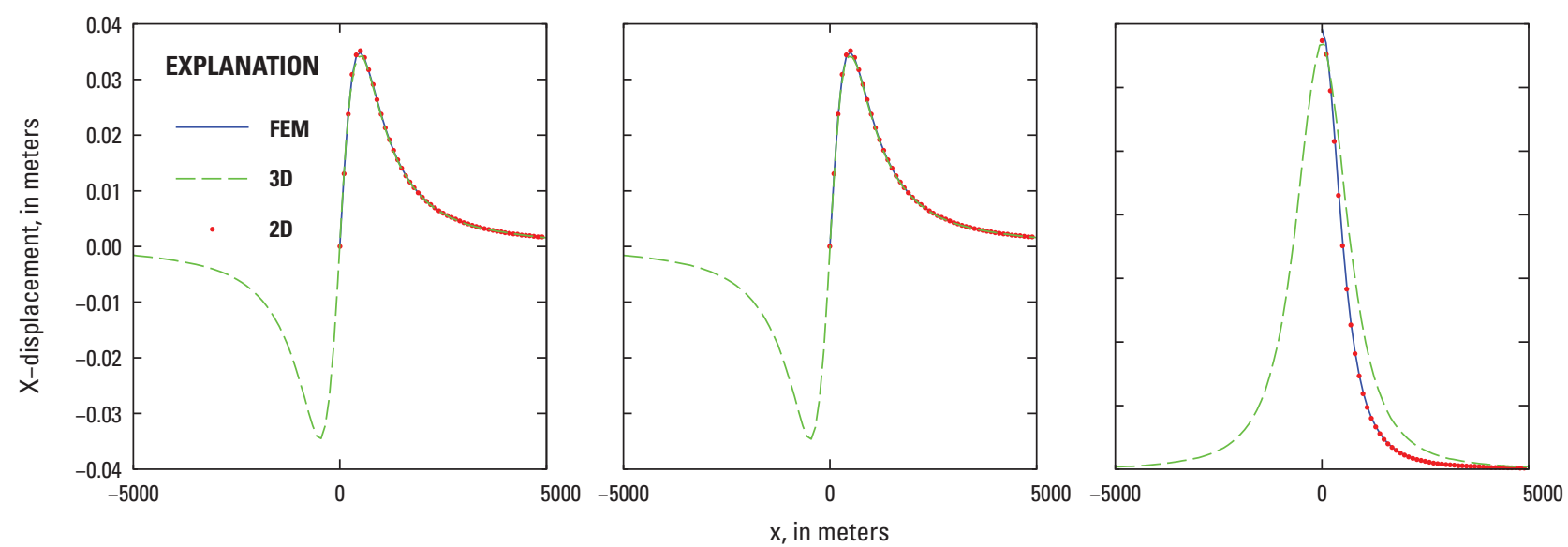

Figure 3. Free-surface deformation caused by a pressurized spherical magma chamber. Comparison between the three-dimensional (3D) semianalytical solution (equation 22), the two-dimensional (2D) analytical model (equations 16 and 17), and a numerical finite element method (FEM) model of the same source. The source is at the origin of the coordinate system, and its parameters are $a=500$ meters, $z_{0}=2 a=1,000$ meters, and $\Delta P / \mu=0.001273$. 


\section{Volume Change}

The change in the volume of the sphere is

$$
\Delta V=\pi a^{3} \frac{\Delta P}{\mu}\left[1+\left(\frac{a}{z_{0}}\right)^{4}\right] .
$$

When $\left(a / z_{0}\right)^{4}$ is much smaller than 1 , equation 19 will return the well known result for the volume change caused by a pressurized point source (Amoruso and Crescentini, 2009; fig. 4).

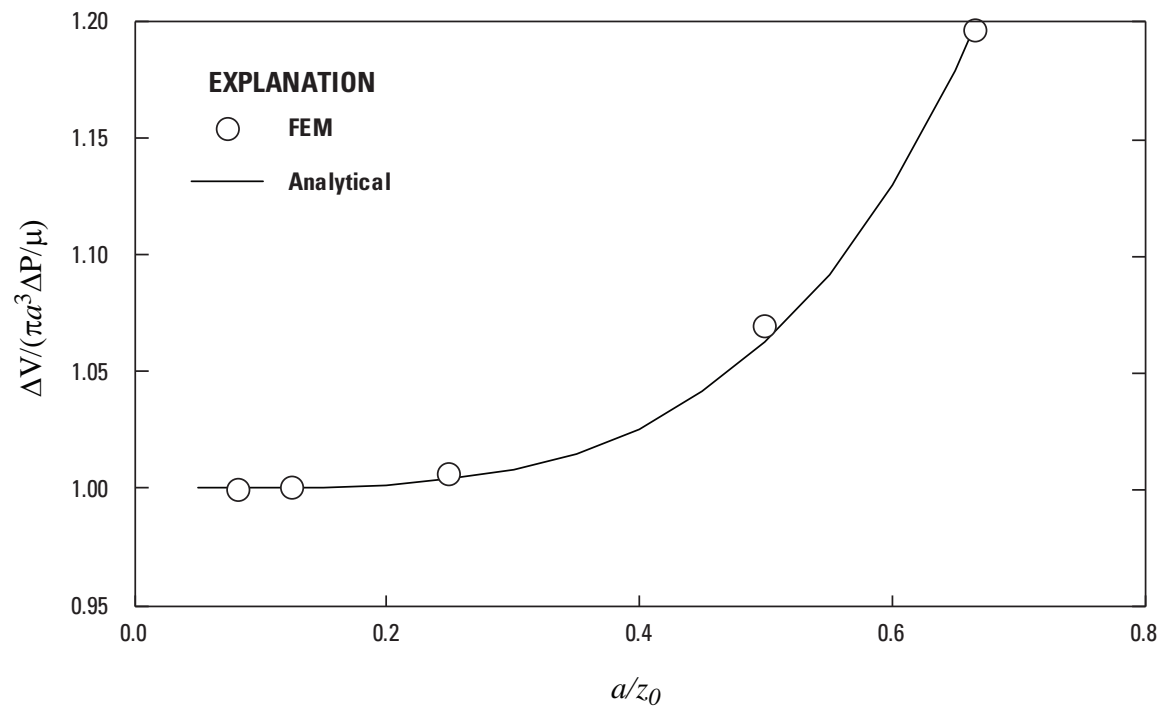

Figure 4. Comparison between the dimensionless volume change $\left(\Delta V /\left(\pi a^{3} \Delta P / \mu\right)\right)$ of a pressurized sphere calculated by use of equation 19 (analytical, solid line) and a finite element method numerical model (FEM, open circles). $a / z_{0}$ is the ratio of the radius of the magma chamber to the depth of the centroid of the chamber below the free surface. Source parameters for the FEM model are $a=1,000$ meters and $\Delta P / \mu=0.001273$.

\section{Ground Tilt}

Measuring tiny changes in the slope angle or tilt of the ground near a volcano is one of the oldest methods for monitoring deformation caused by moving magma. When magma forces the ground upward, the slope of adjacent areas will usually tilt away from the center of uplift by a small amount. Conversely, if the ground subsides as a consequence of depressurization of a magma body, the slope of adjacent areas will tilt toward the center of subsidence. The components of the tilt vector are the horizontal derivatives of the vertical deformation $u_{z}$ (fig. 5; see table 7 at the end of chapter for numerical example).

$$
\begin{aligned}
& \frac{d u_{z}}{d x}=-(1-v) \frac{\Delta P a^{3}}{\mu} \frac{x z_{0}}{\left(r^{2}+z_{0}^{2}\right)^{5 / 2}}\left\{3-\frac{1}{7-5 v}\left(\frac{a}{z_{0}}\right)^{3}\left[1.5(1+v)-18.75(2-v) \frac{z_{0}^{2}}{r^{2}+z_{0}^{2}}\right]\right\} \\
& \frac{d u_{z}}{d y}=-(1-v) \frac{\Delta P a^{3}}{\mu} \frac{y z_{0}}{\left(r^{2}+z_{0}^{2}\right)^{5 / 2}}\left\{3-\frac{1}{7-5 v}\left(\frac{a}{z_{0}}\right)^{3}\left[1.5(1+v)-18.75(2-v) \frac{z_{0}^{2}}{r^{2}+z_{0}^{2}}\right]\right\}
\end{aligned} .
$$




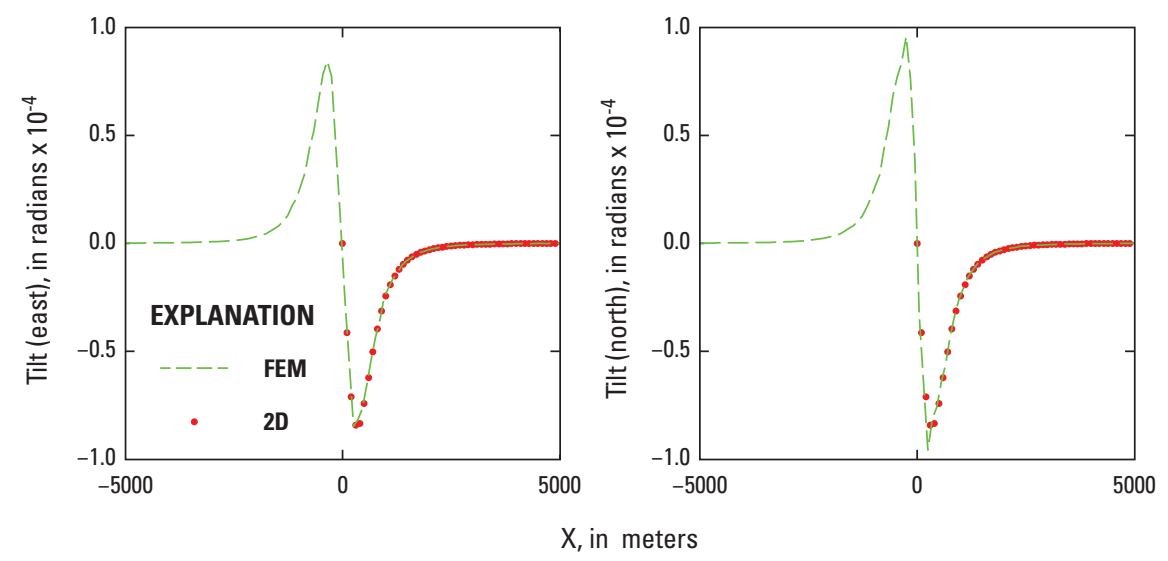

Figure 5. Comparison between calculations of ground tilt in the east and north direction by a two-dimensional (2D) analytical model (equation 20), and a finite element method (FEM) numerical model. As in figure 3, source parameters are $a=500$ meters, $z_{0}=2 a=1,000$ meters, and $\Delta P / \mu=0.001273$.

\section{Internal Deformation and Strain}

The internal deformation and strain is better described using the dimensionless notation shown in fig. 6 .The spatial coordinates are scaled by the depth of the spherical source $z_{0}$. The radius of the spherical cavity is defined by $\varepsilon=a / z_{0}$. The deformation is scaled by $z_{0}$, the pressure change $\Delta P$, and the shear modulus $\mu$

$$
\begin{aligned}
& \xi=\frac{x-x_{0}}{z_{0}} \quad \psi=\frac{y-y_{0}}{z_{0}} \quad \rho=\xi^{2}+\psi^{2} \\
& \frac{u_{z}^{(3 D)}}{z_{0}} \frac{\mu}{\Delta P}=\bar{u}_{\zeta}^{(3 D)} \quad \frac{u_{r}^{(3 D)}}{z_{0}} \frac{\mu}{\Delta P}=\bar{u}_{\rho}^{(3 D)}=\rho \bar{u}_{\zeta}^{(3 D)}
\end{aligned},
$$

The internal deformation caused by a spherical source is given by the superposition of a leading-order solution $\bar{u}^{(0)}$, a first free-surface correction $\bar{u}^{(1)}$, a higher order cavity correction $\bar{u}^{(3)}$, and a sixth-order free-surface correction $\bar{u}^{(6)}$ (see tables 7 and 9 at the end of chapter for numerical examples):

$$
\begin{aligned}
& \bar{u}_{\rho}^{(3 D)}=\bar{u}_{\rho}^{(0)}+\varepsilon^{3} \bar{u}_{\rho}^{(1)}+\varepsilon^{3} \bar{u}_{\rho}^{(3)}+\varepsilon^{6} \bar{u}_{\rho}^{(6)} \\
& \bar{u}_{\zeta}^{(3 D)}=\bar{u}_{\zeta}^{(0)}+\varepsilon^{3} \bar{u}_{\zeta}^{(1)}+\varepsilon^{3} \bar{u}_{\zeta}^{(3)}+\varepsilon^{6} \bar{u}_{\zeta}^{(6)}
\end{aligned} .
$$

At the free surface ( $\zeta=0$ ), equations return equations 16 and 17 .

\section{Leading-Order Solution}

The internal displacements in cylindrical coordinates system are

$$
\begin{aligned}
& \bar{u}_{\rho}^{(0)}=\varepsilon^{3} \frac{1}{4} \frac{\rho}{\left[\rho^{2}+(1-\zeta)^{2}\right]^{3 / 2}} . \\
& \bar{u}_{\zeta}^{(0)}=\varepsilon^{3} \frac{1}{4} \frac{1-\zeta}{\left[\rho^{2}+(1-\zeta)^{2}\right]^{3 / 2}}
\end{aligned}
$$

At the free surface ( $\zeta=0$ ), equations return McTigue's (1987) equations 14 and 15. 
$\boldsymbol{A}$

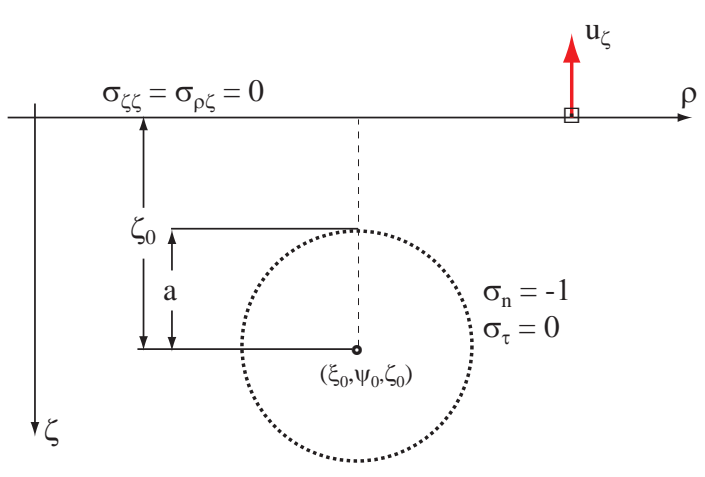

B

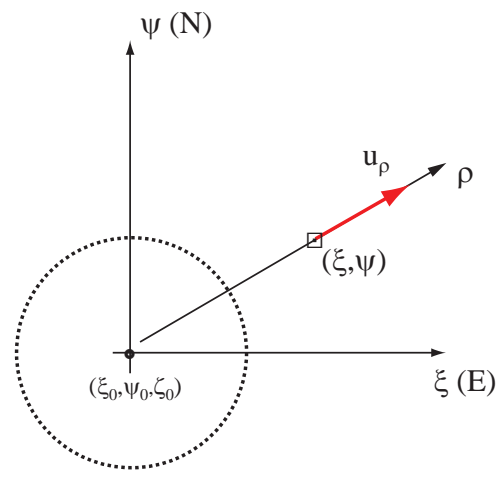

Figure 6. Definition of dimensionless boundary conditions and coordinates for a spherical source. $A$, Vertical cross section showing the boundary conditions: the normal stresses $\sigma_{\rho \zeta}$ and $\sigma_{\zeta \zeta}$ are equal to zero at the free surface, whereas the normal stress $\sigma_{n}$ is equal -1 at the surface of the spherical magma chamber, and the tangential stress $\sigma_{\tau}$ is zero. $\rho$ and $\zeta$ are local cylindrical coordinates, $a$ is the radius of the spherical source, $\left(\xi_{0}, \psi_{0}, \zeta_{0}\right)$ is the source location, $u_{\zeta}$ is the uplift. $B$, The local coordinate system at the earth's surface. $\xi$ (east) and $\psi$ (north) are local Cartesian coordinates, $u_{\rho}$ is the radial displacement.

\section{First Free-Surface Correction}

The displacements for the first-order free-surface correction are given by the appropriate combination of the following Hankel transforms (McTigue, 1987, p. 12939)

$$
\begin{aligned}
& u_{\rho}(\rho, \zeta)=\frac{1}{2} \int_{0}^{\infty} \bar{\sigma}[(1-2 v)-t \zeta] e^{-t \zeta} J_{1}(t \rho) d t \\
& u_{\rho}(\rho, \zeta)=\frac{1}{2} \int_{0}^{\infty} \bar{\tau}_{1}[2(1-v)-t \zeta] e^{-t \zeta} J_{1}(t \rho) d t \\
& u_{\zeta}(\rho, \zeta)=\frac{1}{2} \int_{0}^{\infty} \bar{\sigma}[2(1-v)-t \zeta] e^{t \zeta} J_{0}(t R) d t \\
& u_{\zeta}(\rho, \zeta)=\frac{1}{2} \int_{0}^{\infty} \bar{\tau}_{1}[(1-2 v)-t \zeta] e^{t \zeta} J_{0}(t R) d t
\end{aligned}
$$

where $\bar{\sigma}=\bar{\tau}_{1}=\frac{1}{2} t e^{-t}$ and $R=\sqrt{\rho^{2}+\zeta^{2}}$. The integrals in equation 24 can be solved numerically. At the free surface ( $\zeta=0$ ), equations return McTigue's (1987) equations 24 and 25 (fig. 7; see table 8 at the end of chapter for numerical examples). 


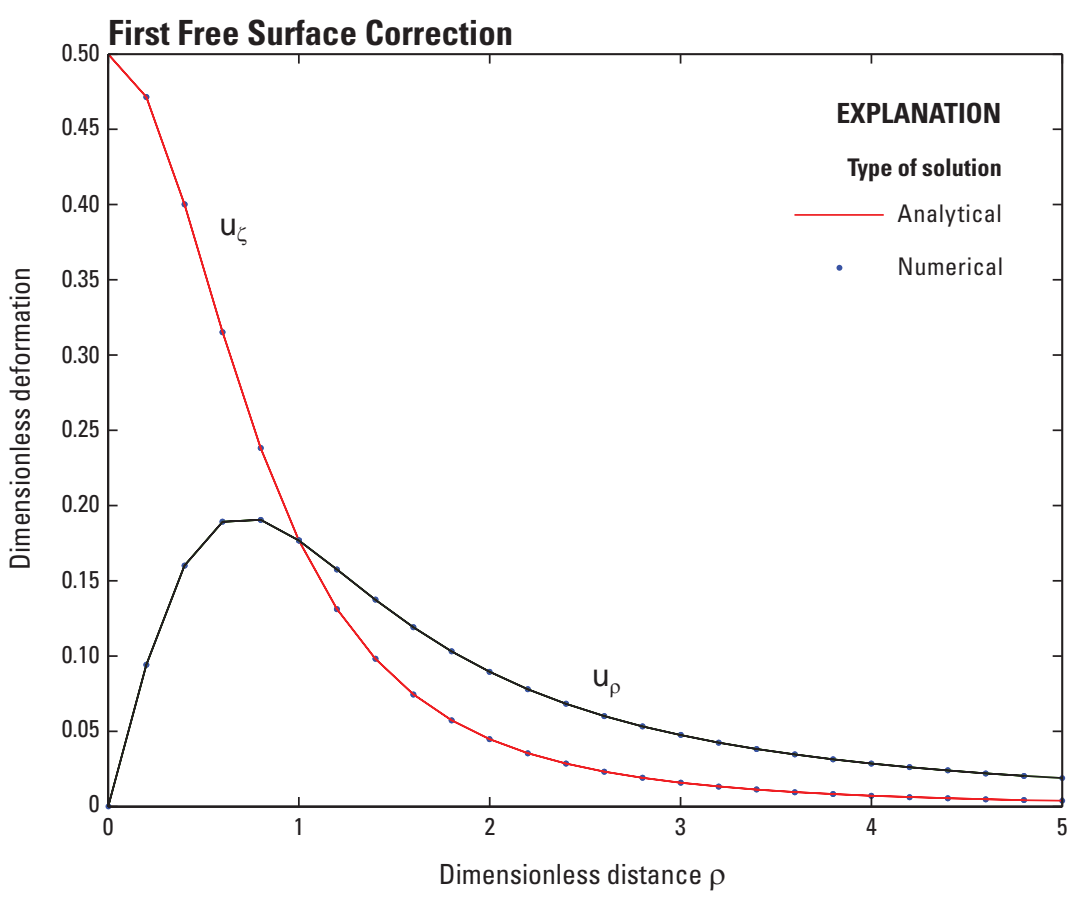

Figure 7. First free-surface correction. Blue points, numerical solution of equation 24 at $\zeta=0$; red/green line, analytical representation of free-surface deformation, equations 16 and 17. $u_{\rho}$, dimensionless radial deformation; $u_{\zeta}$, dimensionless vertical deformation.

\section{Higher Order Cavity Correction}

The displacements for the higher order cavity correction are given by

$$
\begin{aligned}
& \bar{u}_{\rho}^{(3)}=u_{R} \sin \theta+u_{\theta} \cos \theta \\
& \bar{u}_{\zeta}^{(3)}=u_{R} \cos \theta-u_{\theta} \sin \theta
\end{aligned}
$$

where

$$
\begin{aligned}
\sin \theta & =\frac{\rho}{R} ; \quad \cos \theta=\frac{1-\zeta}{R} . \\
R & =\sqrt{\rho^{2}+(1-\zeta)^{2}}
\end{aligned}
$$

McTigue's (1987) equations 38-41 provide these expressions for $u_{R}$ and $u_{\theta}$ :

$$
\begin{gathered}
u_{R}=-\frac{1}{2} \frac{D_{0}^{(3)}}{R^{2}} P_{0}+\left[C_{2}^{(3)}(5-4 v)-\frac{3}{2} \frac{D_{2}^{(3)}}{R^{2}}\right] \frac{P_{2}}{R^{2}}, \\
u_{\theta}=-\left[C_{2}^{(3)}(1-2 v)+\frac{1}{2} \frac{D_{2}^{(3)}}{R^{2}}\right] \frac{1}{R^{2}} \frac{d P_{2}}{d \theta}
\end{gathered}
$$

where the $C$ and $D$ coefficients are

$$
C_{2}^{(3)}=5 \varepsilon^{3} \frac{2-v}{25(7-5 v)}, \quad D_{0}^{(3)}=-\varepsilon^{3} \frac{1+v}{12} \text { and } \quad D_{2}^{(3)}=\varepsilon^{5} \frac{2-v}{4(7-5 v)}
$$


and the Legendre's polynomial and derivatives are

$$
P_{0}=1, \quad P_{2}=\frac{1}{2}\left(3 \cos ^{2} \theta-1\right) \text { and } \frac{d P_{2}}{d \theta}=3 \cos \theta .
$$

Equation 25 return McTigue's (1987) equations 46 and 47 (fig. 8) at the the free surface $(\zeta=0)$.

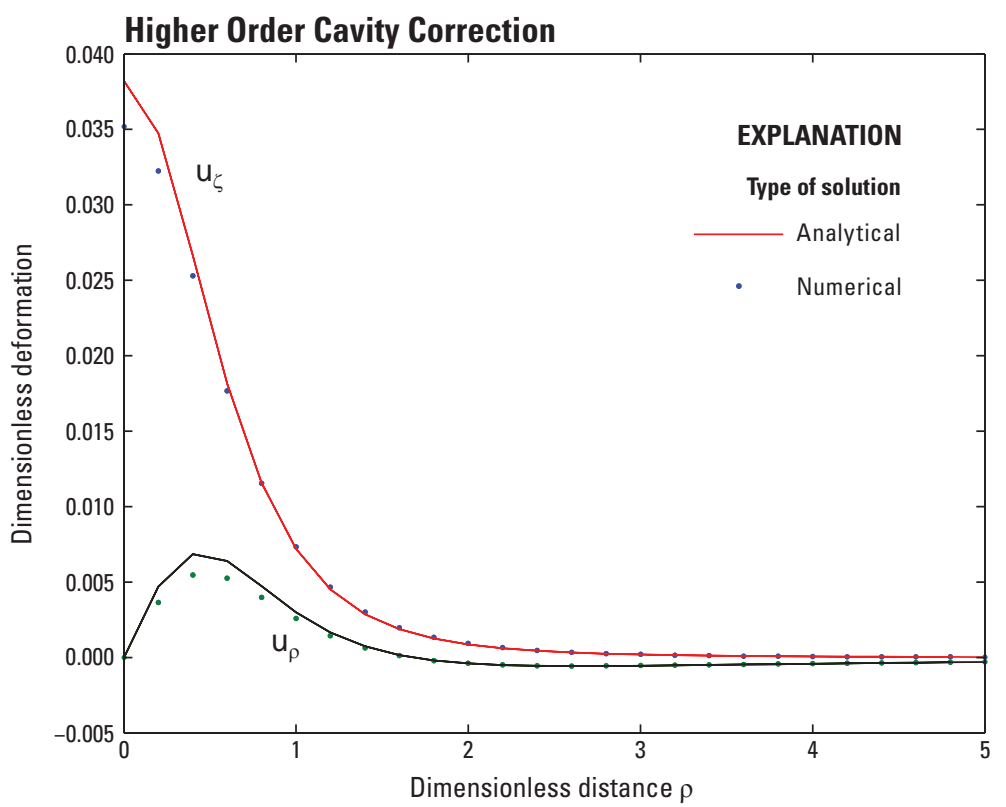

Figure 8. Higher order cavity correction at the free surface, $\zeta=0$. Blue points, numerical solution of equation 25 at $\zeta=0$; red/green line, analytical representation of surface deformation, equations 16 and 17. $u_{\rho}$, dimensionless radial deformation; $u_{\zeta}$, dimensionless vertical deformation.

\section{Sixth-Order Free-Surface Correction}

The procedure to determine the sixth-order free-surface correction is identical to that for obtaining the first-order surface effect (equation 24). The following expressions describe the normal and shear stresses at the free surface:

$$
\begin{aligned}
& u_{\rho} \rightarrow \bar{\sigma} \simeq \frac{3}{2}\left(t+t^{2}\right) \frac{e^{-t}}{7-5 v}, \\
& u_{\zeta} \rightarrow \bar{\sigma} \simeq t^{2} \frac{e^{-t}}{7-5 v}
\end{aligned}
$$

and

$$
\bar{\tau}_{1} \simeq t^{2} \frac{e^{-t}}{7-5 v} .
$$

For $\zeta=0$ and the stresses given by equations 30 and 31, equation 24 returns McTigue's (1987) equations 50 and 51 (fig. 9; see table 8 at the end of chapter for numerical examples). 


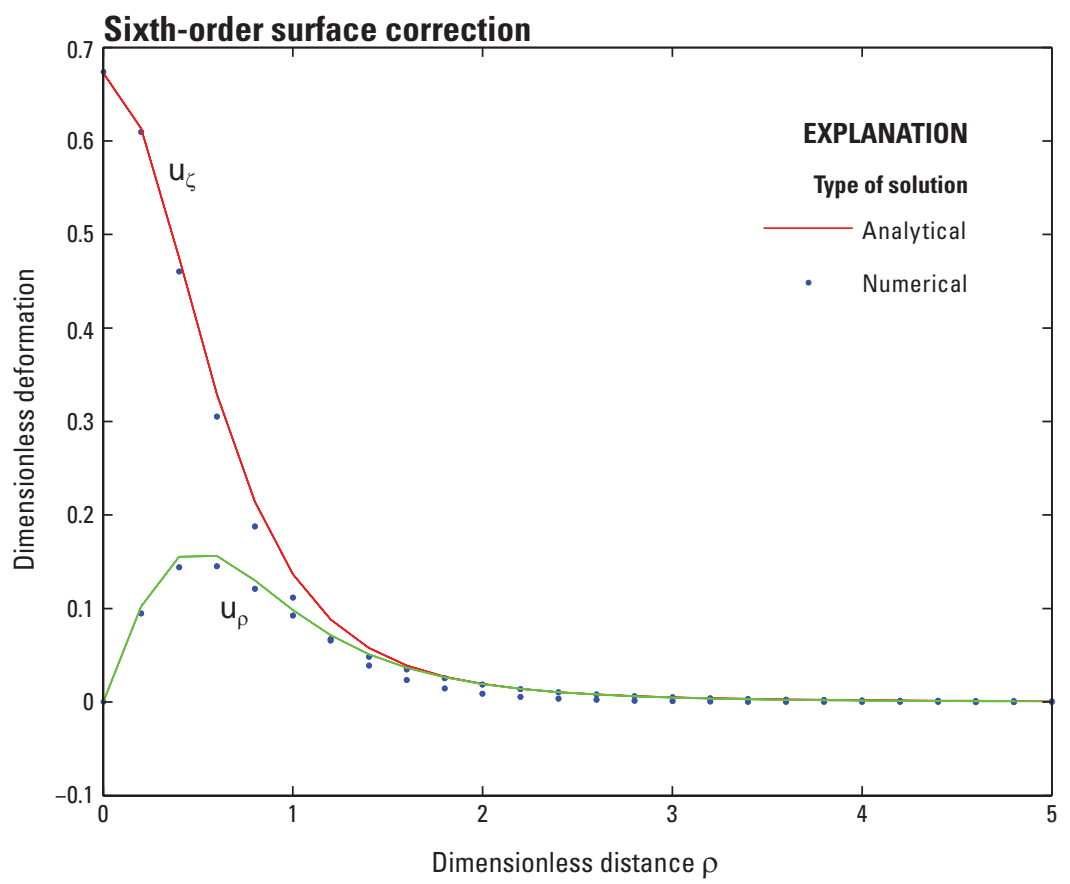

Figure 9. Sixth-order surface correction at $\zeta=0$. Blue points, numerical solution (equations 24,30 , and 31 ) at $\zeta=0$; red/green line, analytical representation of surface deformation, equations 16 and 17. $u_{\rho}$, dimensionless radial deformation; $u_{\zeta}$, dimensionless vertical deformation.

\section{Internal Deformation}

Figure 10 on the next page shows the verification for the expression for internal deformation (equation 22).

\section{Strain}

Strainmeters can resolve changes in strain of less than one part per billion (1 $\mathrm{mm}$ in $1,000 \mathrm{~km}$ ) over short periods, which makes them ideal for capturing transient deformation over time intervals ranging from seconds to months. Gladwin Tensor Strainmeters (GTSMs), the instruments used by the Plate Boundary Observatory (PBO), are designed to measure three components that describe the horizontal strain tensor: the areal strain $\varepsilon_{a}$, and shear strains $\gamma_{1}$ and $\gamma_{2}$. Traditional engineering analysis has led to these quantitative definitions of quantities $\varepsilon_{a}, \gamma_{1}$, and $\gamma_{2}$ :

$$
\begin{aligned}
& \varepsilon_{a}=\varepsilon_{x x}+\varepsilon_{y y}, \quad \gamma_{1}=\varepsilon_{x x}-\varepsilon_{y y}, \quad \text { and } \gamma_{2}=2 \varepsilon_{x y} ; \\
& \varepsilon_{x x}=\frac{\partial u_{x}^{(3 D)}}{\partial x}, \quad \varepsilon_{y y}=\frac{\partial u_{y}^{(3 D)}}{\partial y} \text {, and } \quad \varepsilon_{x y}=\varepsilon_{y x}=\frac{1}{2}\left(\frac{\partial u_{x}^{(3 D)}}{\partial y}+\frac{\partial u_{y}^{(3 D)}}{\partial x}\right)
\end{aligned}
$$

Because of the complexity of the equation 22 for the internal deformation, we compute the strain components shown in equation 32 by use of numerical derivatives (see appendix 1). 

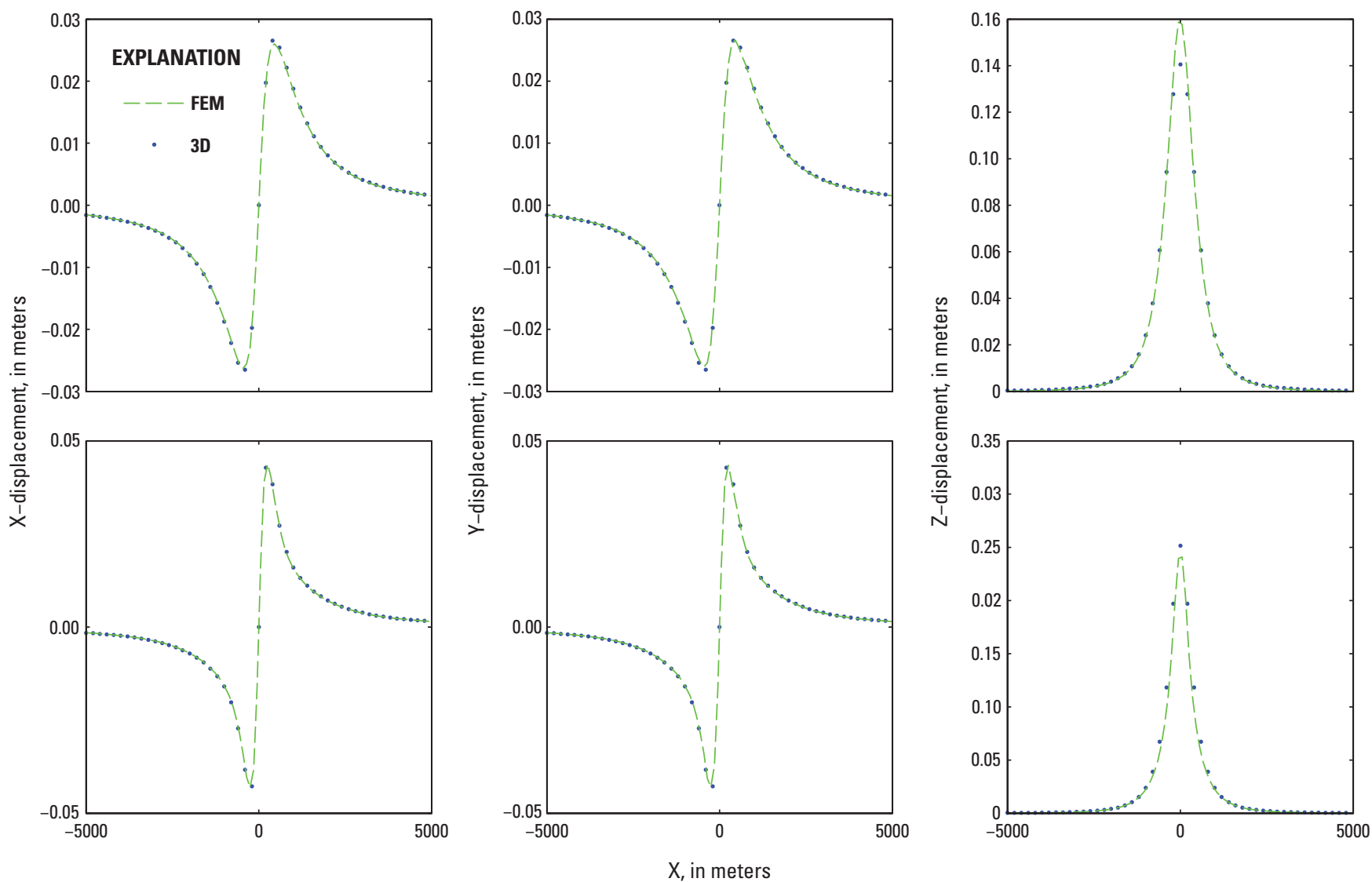

Figure 10. Internal deformation caused by a spherical source. The first row shows internal deformation at $z_{0}=0.5 a$, and the second row internal deformation at $z_{0}=a$. Blue points (3D), analytical solution, equation 22; dashed green line (FEM), finite element method numerical solution. Source parameters for the FEM model are $z_{0}=1,000$ meters and $\Delta P / \mu=0.001273$. It was necessary to change some of the signs of $\zeta$ in the original integrals A7-A18 in McTigue (1987) to improve the fit between the analytical and the numerical solution at depth $\zeta$ greater than zero. 

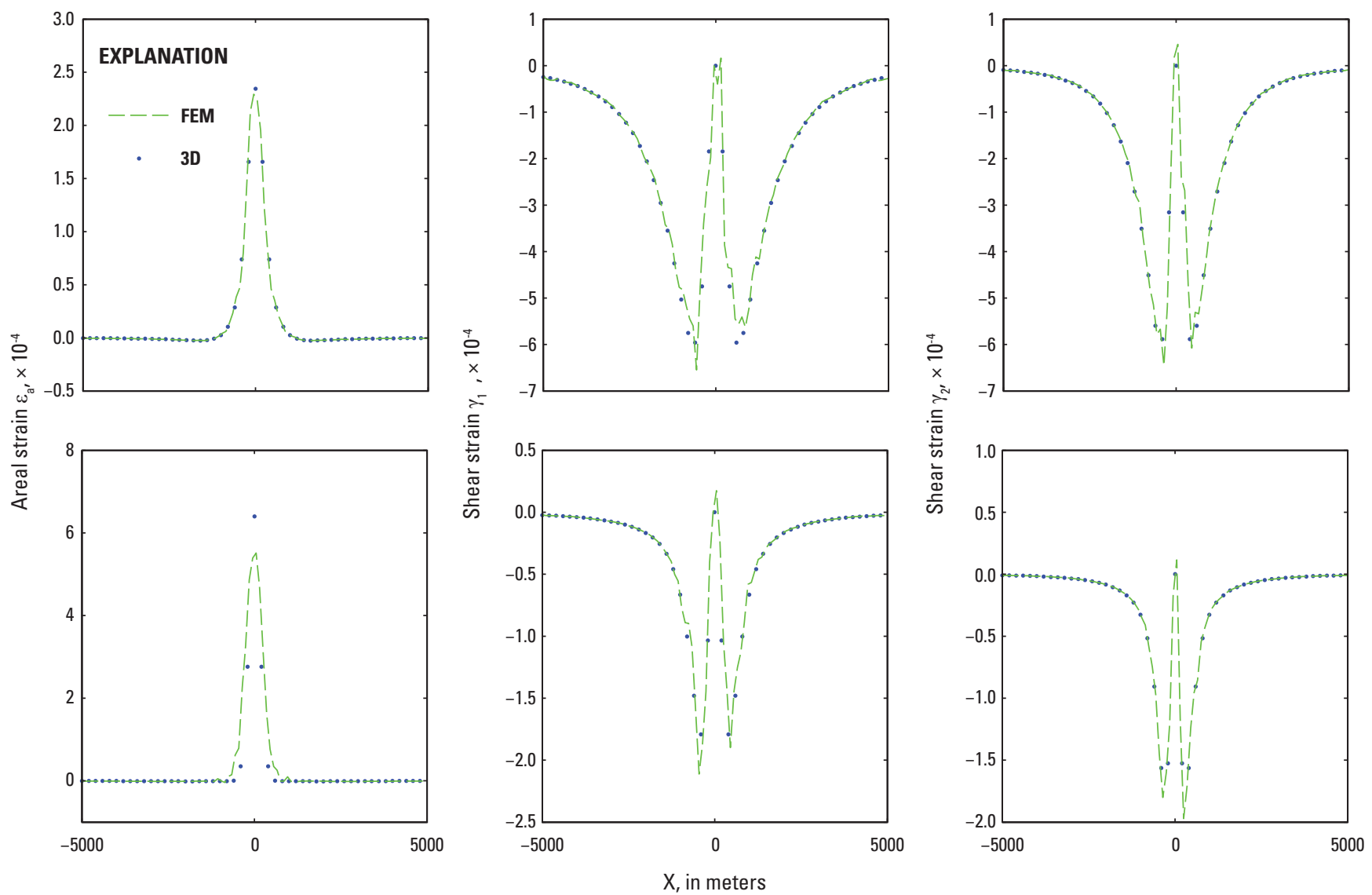

Figure 11. Internal strain caused by a spherical source. The first row shows internal strain at $z_{0}=0.5 a$, and the second row internal strain at $z_{0}=a$. Blue points (3D), analytical solution, equation 32; dashed green line (FEM), finite element method numerical solution. Source parameters for the FEM model are $z_{0}=1,000$ meters and $\Delta P / \mu=0.001273$. 


\section{Examples of Surface Deformation, Tilt, Internal Deformation, and Strain for a Spherical Source}

Table 7. Surface deformation, tilt, and internal deformation.

[Surface deformation, tilt and internal deformation at $\mathrm{z}=0$ from equations 18, 20, and 22. See figures 3 and 5 . Parameters are $a=500$ meters, $z_{0}=2 a=1,000$ meters, and $\Delta P / \mu=0.001273$. $x$ and $y$, local Cartesian coordinates; $u_{x}, u_{y}$, and $u_{z}$ displacements at the free surface $\partial u_{z} / \partial x$ and $\partial u_{z} / \partial y$, tilt components; $u_{x}^{(3 D)}, u_{y}^{(3 D)}$, and, $u_{z}^{(3 D)}$, internal deformation]

\begin{tabular}{|c|c|c|c|c|c|c|c|c|c|}
\hline $\begin{array}{c}x \\
\left(10^{3} \text { meters }\right)\end{array}$ & $\begin{array}{c}y \\
\left(10^{3} \text { meters }\right)\end{array}$ & $\begin{array}{c}u_{x} \\
\left(10^{-3} \text { meters }\right)\end{array}$ & $\begin{array}{c}u_{v} \\
\left(10^{-3} \text { meters }\right)\end{array}$ & $\begin{array}{c}u_{z} \\
\left(10^{-3} \text { meters }\right)\end{array}$ & $\begin{array}{l}d u_{z} / d x \\
\left(\times 10^{-6}\right)\end{array}$ & $\begin{array}{c}d u_{z} / d y \\
\left(\times 10^{-6}\right)\end{array}$ & $\begin{array}{c}u_{x}^{(3 D)} \\
\left(10^{-3} \text { meters }\right)\end{array}$ & $\begin{array}{c}u_{y}^{(3 \mathrm{D})} \\
\left(10^{-3} \text { meters }\right)\end{array}$ & $\begin{array}{c}u_{z}^{(3 D)} \\
\left(10^{-3} \text { meters }\right)\end{array}$ \\
\hline-4.0 & -4.0 & -2.4949 & -2.4949 & 0.6237 & 0.2275 & 0.2275 & -2.4973 & -2.4973 & 0.6305 \\
\hline 0.0 & 0.0 & 0.0000 & 0.0000 & 134.7482 & 0.0000 & 0.0000 & 0.0000 & 0.0000 & 138.3455 \\
\hline 2.0 & 2.0 & 8.8603 & 8.8603 & 4.4301 & 2.9846 & 2.9846 & 8.8631 & 8.8631 & 4.4876 \\
\hline
\end{tabular}

Table 8. Corrections for internal deformation.

[Corrections at $\zeta=0$ from equations 24, 25, 30, and 31. See figures 7 through 9. Parameters are $a=500$ meters, $z_{0}=2 a=1,000$ meters and $\underset{{ }^{\prime}}{\Delta P} / \mu=0.001273 . \rho$, dimensionless distance, equation $21 ; u_{r}^{(1)}$ and $u_{z}^{(1)}$, first-order correction; $u_{r}^{(3)}$ and $u_{z}^{(3)}$, higher order correction; $u_{r}^{(6)}$ and , $u_{z}^{(6)}$ sixth-order correction]

\begin{tabular}{|c|c|c|c|c|c|c|}
\hline$\rho$ & $u_{r}^{(1)}\left(X 10^{-3}\right)$ & $u_{z}^{(1)}\left(X 10^{-3}\right)$ & $u_{r}^{(3)}\left(\times 10^{-3}\right)$ & $u_{z}^{(3)}\left(X 10^{-3}\right)$ & $u_{r}^{(6)}\left(X 10^{-3}\right)$ & $u_{z}^{(6)}\left(X 10^{-3}\right)$ \\
\hline 0.0 & 0.0000 & 500.0000 & 0.0000 & 35.1751 & 0.0000 & 673.9130 \\
\hline 2.0 & 89.4426 & 44.7213 & -0.3950 & 0.9202 & 18.6663 & 8.9443 \\
\hline
\end{tabular}

Table 9. Internal deformation and strain.

[Internal deformation and strain ( $\mathrm{z}=\mathrm{a}=500$ meters) from equations 22 and 32. See figures 10 and 11. Parameters are $a=500$ meters, $z_{0}=2 a=1,000$ meters and $\Delta P / \mu=0.001273$. $\mathrm{x}$ and $\mathrm{y}$, local Cartesian coordinates; $u_{x}{ }^{(3 D)}, u_{y}{ }^{(3 D}$ 'and $u_{z}{ }^{(3 D)}$, internal deformation; $\varepsilon_{a}, \gamma_{1}$ and $\gamma_{2}$, strain. The strain component $\gamma_{1} \equiv 0$ for $x=y$ ]

\begin{tabular}{|c|c|c|c|c|c|c|c|c|c|}
\hline $\begin{array}{c}x \\
\left(X 10^{3} \text { meters }\right.\end{array}$ & $\begin{array}{c}y \\
\left(X 10^{3} \text { meters }\right)\end{array}$ & $\underset{\left(10^{-3} \underset{x}{u^{(3 D)}}\right.}{\text { meters })}$ & $\underset{\left(10^{-3}{ }_{\text {meters }}^{u^{(3 \mathrm{D})}}\right.}{ }$ & $\underset{\left(10^{-3} \text { meters }\right)}{u_{z}^{(3 \mathrm{D})}}$ & $\begin{array}{c}\varepsilon_{a} \\
\left(X 10^{-6}\right)\end{array}$ & $\left(\begin{array}{c}\gamma_{2} \\
\left(\mathbf{x}^{-6}\right)\end{array}\right.$ & $\begin{array}{c}x \\
\left(X 10^{3} \text { meters }\right)\end{array}$ & $\begin{array}{c}y \\
\left(X 10^{3} \text { meters }\right)\end{array}$ & $\left.\begin{array}{c}\gamma_{1} \\
\left(\times 10^{-6}\right.\end{array}\right)$ \\
\hline-4.0 & -4.0 & -2.3320 & -2.3320 & 0.5283 & -0.4610 & -1.6271 & -4.0 & 0.0 & -3.9171 \\
\hline 2.0 & 2.0 & 7.1229 & 7.1229 & 3.9175 & -1.3665 & -8.4895 & 2.0 & 0.0 & -16.7005 \\
\hline
\end{tabular}




\section{Prolate Spheroid Source (Magma Conduit)}

A simple model of a volcanic system includes two principal elements: a magma reservoir and a conduit through which magma may reach the surface. When the volcano is quiescent, the conduit will close, allowing pressure to build up in the reservoir. Yang and others $(1988)^{1}$ formulated an approximate but accurate half-space double-force and center-of-dilatation solutions for a dipping prolate ellipsoid in an elastic half-space. The general solution for a prolate spheroid depends on seven parameters (fig. 12): the dimensionless pressure change $\Delta P / \mu$, the geometric aspect ratio $A=b / a$ between the semimajor axis $a$ and the semiminor axis $b$, the source location $\left(x_{0}, y_{0}, z_{0}\right)$, the dip angle $\theta$ (measured from the free surface), and the strike (or azimuth) angle $\phi$ (measured clockwise from the positive North direction).

The expressions for the three components of deformation in the near field are quite complex. Coding the expressions for near-field displacement, tilt, and strain required writing the four nested functions described below (fig. 13). The formulas are introduced in the same order as their use in the corresponding Matlab functions.
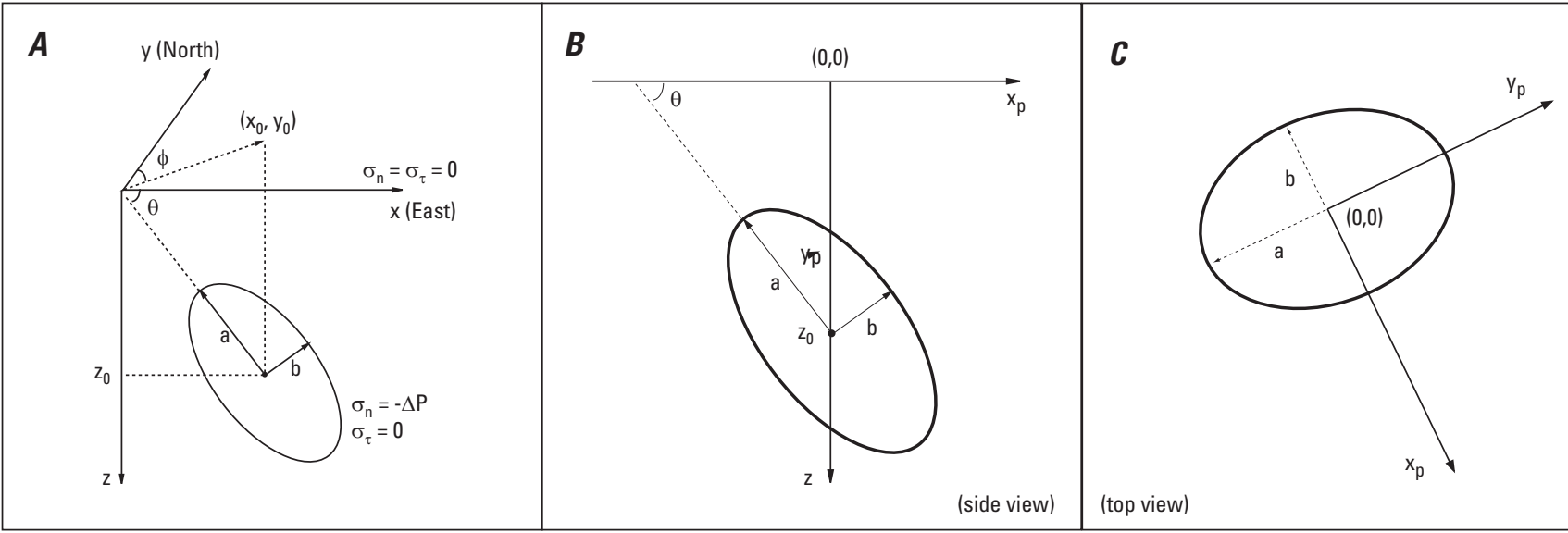

Figure 12. Diagrams showing $A$, definition of coordinates system and boundary-value problem for a spheroidal source. $x, y$ and $z$, local Cartesian coordinates; $x_{0^{\prime}} y_{0}$ and $z_{0^{\prime}}$ source location; $\theta$, dip angle; $\phi$, strike (or azimuth) angle; $a$, semimajor axis; $b$, semiminor axis; the normal stress $\sigma_{n}$ and shear stress $\sigma_{\tau}$ are equal to zero at the free surface, whereas the normal stress $\sigma_{n}$ is equal to the pressure change at the surface of the spherical magma chamber, and the shear stress $\sigma_{\tau}$ is zero. $B$ and $C$, coordinate system used by Yang and others (1988). $x_{p^{\prime}} y_{p}$ and $z$, local Cartesian coordinate system; the source centroid is at the origin of the coordinate system, and the strike $\phi$ is equal to zero.

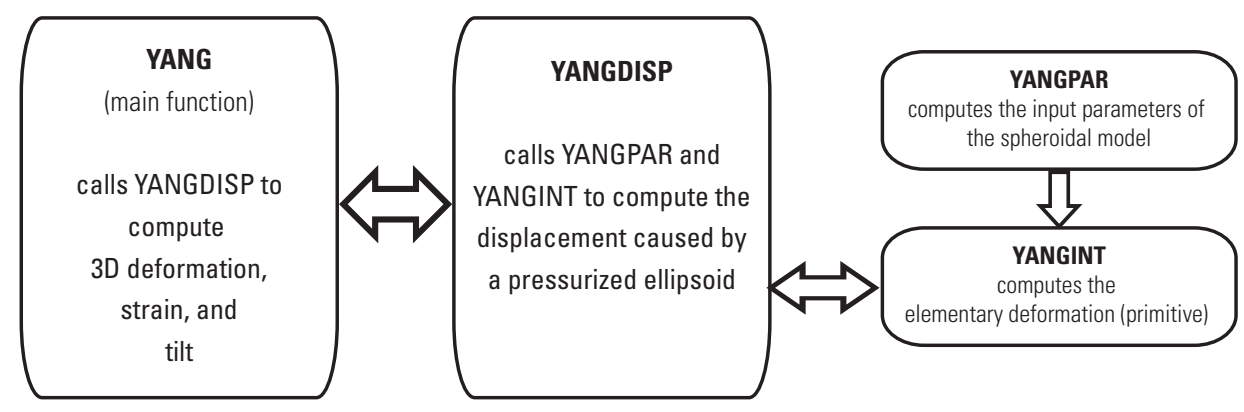

Figure 13. Flow chart for coding Yang and others' (1988) expressions for displacement, tilt, and strain.

\footnotetext{
${ }^{1}$ Corrections to Yang and others' (1988) equations are reported in Newman and others (2006), and in this publication.
} 


\section{Coordinates and Displacement (YANGDISP)}

To compute the displacement, coordinates must be transformed from the Cartesian coordinate system (UTM) onto the coordinate system used by Yang and others (1988). The displacement vectors then can be back-transformed onto the original system (fig. 12).

We first translate and rotate the coordinates

$$
\begin{aligned}
& x_{p}=\cos \phi\left(x-x_{0}\right)-\sin \phi\left(y-y_{0}\right), \\
& y_{p}=\sin \phi\left(x-x_{0}\right)+\cos \phi\left(y-y_{0}\right)
\end{aligned}
$$

and then we compute the general expressions

$$
\begin{aligned}
U p_{x} & =-U_{1}(\xi=c)-U_{1}(\xi=-c) \\
U p_{y} & =-U_{2}(\xi=c)-U_{2}(\xi=-c) \\
U p_{z} & =U_{3}(\xi=c)+U_{3}(\xi=-c)
\end{aligned}
$$

for the displacement in Yang and others' (1988) coordinate system, where

$$
\begin{gathered}
U_{i}=U_{i}^{*}+U_{i}^{\dagger} \quad i=1,2,3 \\
U_{i}=U_{i}\left(x_{p}, y_{p}, z, z_{0}, \theta, a_{1}, b_{1}, a, b, \xi, \mu, v, P^{\dagger}\right)
\end{gathered}
$$

are the primitive of the displacement for a prolate ellipsoid. The formulas for $U_{i}^{*}$ and $U_{i}^{\dagger}$ are given by equations 49 and 50. Finally, the displacement vector $\mathbf{U p}$ is back-transformed onto the original coordinate system (see figs. 14 and 15 in the Verification section, p. 25):

$$
\begin{aligned}
& u_{x}=\cos \phi \cdot U p_{x}+\sin \phi \cdot U p_{y} \\
& u_{y}=-\sin \phi \cdot U p_{x}+\cos \phi \cdot U p_{y} . \\
& u_{z}=U p_{z}
\end{aligned}
$$

\section{Primitive (YANGINT)}

To compute the primitive $U_{i}^{*}$ and $U_{i}^{\dagger}$, we must first introduce new coordinates and parameters (see Yang and others, 1988, p. 4251):

$$
\begin{aligned}
& \xi_{2}=\xi \cos \theta \quad \xi_{3}=\xi \sin \theta \\
& x_{1}=x_{p} \quad x_{2}=y_{p} \quad x_{3}=z-z_{0} \quad \bar{x}_{3}=z+z_{0} \\
& y_{1}=x_{1} \quad y_{2}=x_{2}-\xi_{2} \quad y_{3}=x_{3}-\xi_{3} \quad \bar{y}_{3}=\bar{x}_{3}+\xi_{3} \\
& r_{2}=x_{2} \sin \theta-x_{3} \cos \theta \quad q_{2}=x_{2} \sin \theta+\bar{x}_{3} \cos \theta \\
& r_{3}=x_{2} \cos \theta+x_{3} \sin \theta \quad q_{3}=-x_{2} \cos \theta+\bar{x}_{3} \sin \theta \\
& \overline{r_{3}}=r_{3}-\xi \quad \bar{q}_{3}=q_{3}+\xi \\
& R_{1}=\sqrt{y_{1}^{2}+y_{2}^{2}+y_{3}^{2}} \quad R_{2}=\sqrt{y_{1}^{2}+y_{2}^{2}+\bar{y}_{3}^{2}}
\end{aligned}
$$

To make the formulas valid for dip angles $\theta$ unequal to $90^{\circ}$ (in other words, a spheroid that is not vertical), we must correct the parameter $C_{0}$ (see Yang and others, 1988, p. 4251, for the original expression):

$$
C_{0}=z_{0} / \sin \theta
$$


To prevent a "divide by zero" warning at the origin, we must add $\varepsilon_{0}=1 \cdot 10^{-15}$ at the denominator in the formula for $\beta$

$$
\beta=\frac{q_{2} \cos \theta+(1+\sin \theta)\left(R_{2}+\bar{q}_{3}\right)}{\cos \theta y_{1}+\varepsilon_{0}} .
$$

Additional parameters used to simplify the expressions for the deformation are

$$
\begin{aligned}
& \overline{d r}_{3}=R_{1}+\bar{r}_{3} \quad \overline{d q}_{3}=R_{2}+\bar{q}_{3} \quad \overline{d y}_{3}=R_{2}+\bar{y}_{3} \\
& \overline{l r}_{3}=\ln \left(R_{1}+\bar{r}_{3}\right) \quad \overline{l q}_{3}=\ln \left(R_{2}+\bar{q}_{3}\right) \quad \overline{l y}_{3}=\ln \left(R_{2}+\bar{y}_{3}\right), \\
& A_{1}^{*}=\frac{a_{1}}{R_{1} \overline{d r}_{3}}+b_{1}\left(\overline{l r}_{3}+\frac{r_{3}+\xi}{\overline{d r}_{3}}\right) \\
& \bar{A}_{1}^{*}=-\frac{a_{1}}{R_{2} \overline{d q}_{3}}-b_{1}\left(\overline{l q}_{3}+\frac{q_{3}-\xi}{\overline{d q}_{3}}\right)^{\prime} \\
& A_{1}=\frac{\xi}{R_{1}}+\overline{l r}_{3} \quad \bar{A}_{1}=\frac{\xi}{R_{2}}-\overline{l q}_{3} \\
& A_{2}=R_{1}-r_{3} \overline{l r}_{3} \quad \bar{A}_{2}=R_{2}-q_{3} \overline{l q}_{3} \text {, and } \\
& A_{3}=\frac{\xi \bar{r}_{3}}{R_{1}}+R_{1} \quad \bar{A}_{3}=\frac{\xi \bar{q}_{3}}{R_{2}}-R_{2} \\
& B^{*}=\left(\frac{a_{1}}{R_{1}}+2 b_{1} A_{2}\right)+(3-4 v)\left(\frac{a_{1}}{R_{2}}+2 b_{1} \bar{A}_{2}\right) \text {. } \\
& B=\frac{\xi\left(\xi+C_{0}\right)}{R_{2}}-\bar{A}_{2}-C_{0} \overline{l q}_{3}
\end{aligned}
$$

The following four parameters $F$ are called in $U_{i}^{*}$ and $U_{i}^{\dagger}$ (see equations 49 and 50). They were modified from the original formulation of Yang and others (1988) to improve the fit with the numerical FEM solution for internal deformation (fig. 16):

$$
\begin{aligned}
& F_{1}^{*}=0 \\
& F_{2}^{*}=0 . \\
& F_{1}=0 \\
& F_{2}=0
\end{aligned}
$$


The parameters $f$ also are called in $U_{i}^{*}$ and $U_{i}^{\dagger}$ (see equations 49 and 50). While the formulas for $f_{1}$ and $f_{2}$ are from Yang and others (1988), the equation for $f_{3}$ was corrected by Newman and others (2006):

$$
\begin{gathered}
f_{1}=\frac{\xi y_{1}}{\overline{d y}_{3}}+\frac{3}{\cos ^{2} \theta}\left(y_{1} \sin \theta \overline{l y}_{3}-y_{1} \overline{l q}_{3}+2 q_{2} \cdot{ }^{*} \tan ^{-1} \beta\right)+2 y_{1} \overline{l q}_{3}-4 \bar{x}_{3} \frac{\tan ^{-1} \beta}{\cos \theta} . \\
f_{2}=\frac{\xi y_{2}}{\overline{d y}_{3}}+\frac{3}{\cos ^{2} \theta}\left(q_{2} \sin \theta \overline{l q}_{3}-q_{2} \overline{l y}_{3}+2 y_{1} \sin \theta \tan ^{-1} \beta+\cos \theta\left(R_{2}-\bar{y}_{3}\right)\right) \cdots, \text { and } \\
-2 \cos \theta \bar{A}_{2}+\frac{2}{\cos \theta}\left(\bar{x}_{3} \overline{l y}_{3}-q_{3} \overline{l q}_{3}\right) \\
f_{3}=\frac{1}{\cos \theta}\left(q_{2} \overline{l q}_{3}-q_{2} \sin \theta \overline{l y}_{3}+2 y_{1} \tan ^{-1} \beta\right)+2 \sin \theta \bar{A}_{2}+q_{3} \overline{l y}_{3}-\xi
\end{gathered}
$$

The following coefficients are used to compute the displacement

$$
c^{*}=\frac{a b^{2} / \xi^{3}}{16 \mu(1-v)} \text { and } \quad c^{\dagger}=2 c^{*} P^{\dagger}
$$

The displacement components are given by equations 2 to 7 of Yang and others (1988). They are the primitive of equation 1 of Yang and others (1988). Compared to the original formulation, we deleted the z-contribution in $U_{2}^{*}$ and $U_{3}^{*}$ to improve the fit to internal deformation computed by use of the FEM model (fig. 16)

$$
\begin{gathered}
U_{1}^{*}=c^{*}\left[A_{1}^{*} y_{1}+(3-4 v) \bar{A}_{1}^{*} y_{1}+F_{1}^{*} y_{1}\right] \\
U_{2}^{*}=c^{*}\left[\sin \theta\left(A_{1}^{*} r_{2}+(3-4 v) \bar{A}_{1}^{*} q_{2}+F_{1}^{*} q_{2}\right)+\cos \theta\left(B^{*}-F_{2}^{*}\right)\right], \\
U_{3}^{*}=c^{*}\left[-\cos \theta\left(A_{1}^{*} r_{2}+(3-4 v) \bar{A}_{1}^{*} q_{2}-F_{1}^{*} q_{2}\right)+\sin \theta\left(B^{*}+F_{2}^{*}\right)\right] \\
U_{1}^{\dagger}=c^{\dagger}\left[\left(A_{1} y_{1}+(3-4 v) \bar{A}_{1} y_{1}+F_{1} y_{1}\right)-4(1-v)(1-2 v) f_{1}\right] \\
U_{2}^{\dagger}=c^{\dagger}\left[\sin \theta\left(A_{1} r_{2}+(3-4 v) \bar{A}_{1} q_{2}+F_{1} q_{2}\right)-4(1-v)(1-2 v) f_{2} \ldots\right. \\
\left.+4(1-v) \cos \theta\left(A_{2}+\bar{A}_{2}\right)+\cos \theta\left(A_{3}-(3-4 v) \bar{A}_{3}-F_{2}\right)\right] . \\
U_{3}^{\dagger}=c^{\dagger}\left[\cos \theta\left(-A_{1} r_{2}+(3-4 v) \bar{A}_{1} q_{2}+F_{1} q_{2}\right)+4(1-v)(1-2 v) f_{3} \cdots\right. \\
\left.+4(1-v) \sin \theta\left(A_{2}+\bar{A}_{2}\right)+\sin \theta\left(A_{3}+(3-4 v) \bar{A}_{3}+F_{2}-2(3-4 v) B\right)\right]
\end{gathered}
$$




\section{Modeling Crustal Deformation near Active Faults and Volcanic Centers-A Catalog of Deformation Models}

\section{General Parameters (YANGPAR)}

The general parameters necessary to compute the displacement for a prolate spheroid are listed in table 10 in the same order as their use in the code.

Table 10. General parameters.

[after Yang and others, 1988, p. 4250]

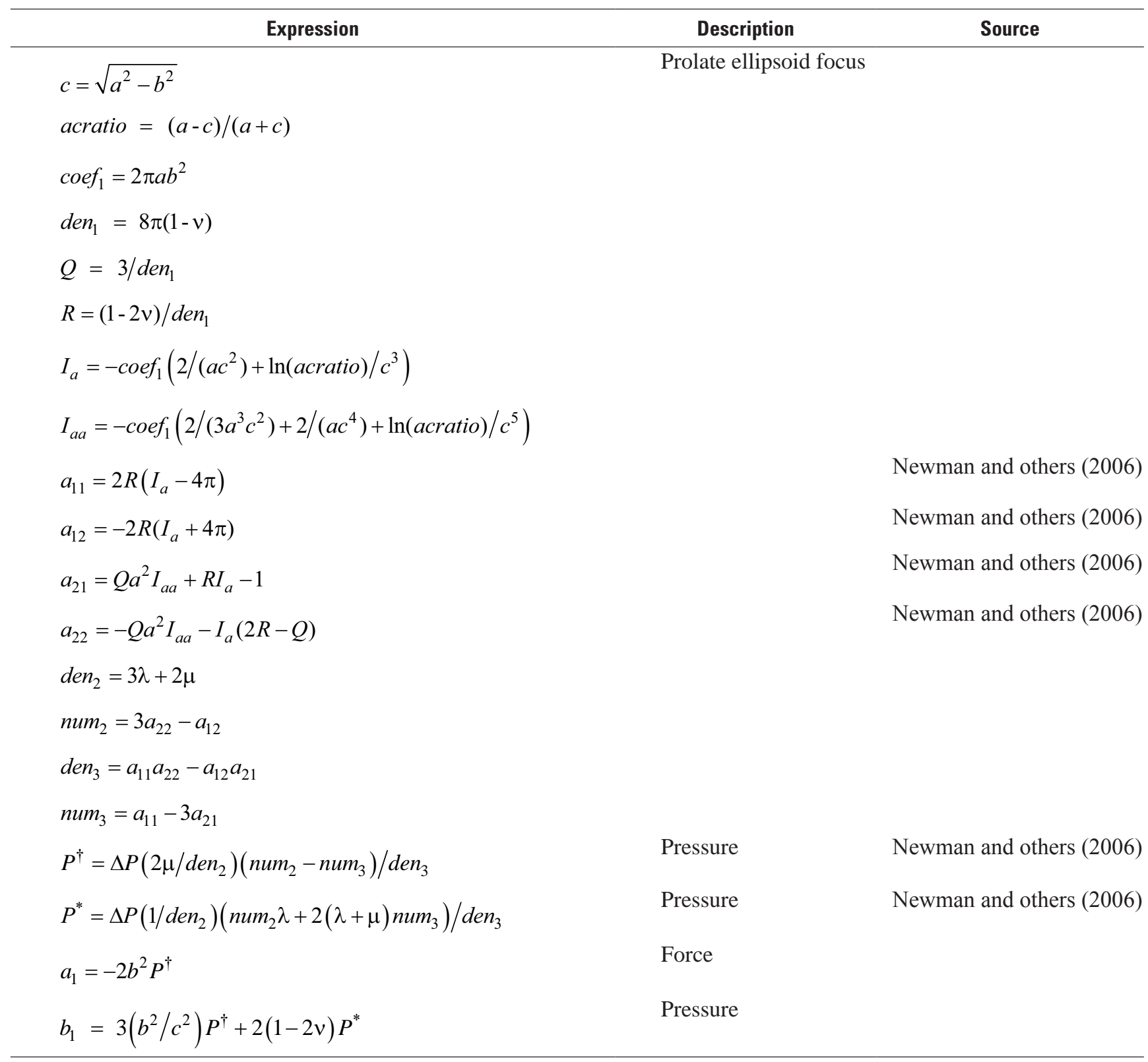




\section{Verification}

We tested the complete derivation by comparing analytical results to three-dimensional FEM solutions for vertical, tilted, and rotated prolate spheroids (figs. 14 and 15).

Results for the internal deformation are shown in figure 16. Although there is no significant difference between our formulation and that of Yang and others (1988) for shallow depths, our formulation provides a better fit to the FEM solution for greater depths (fig. 16, third row).
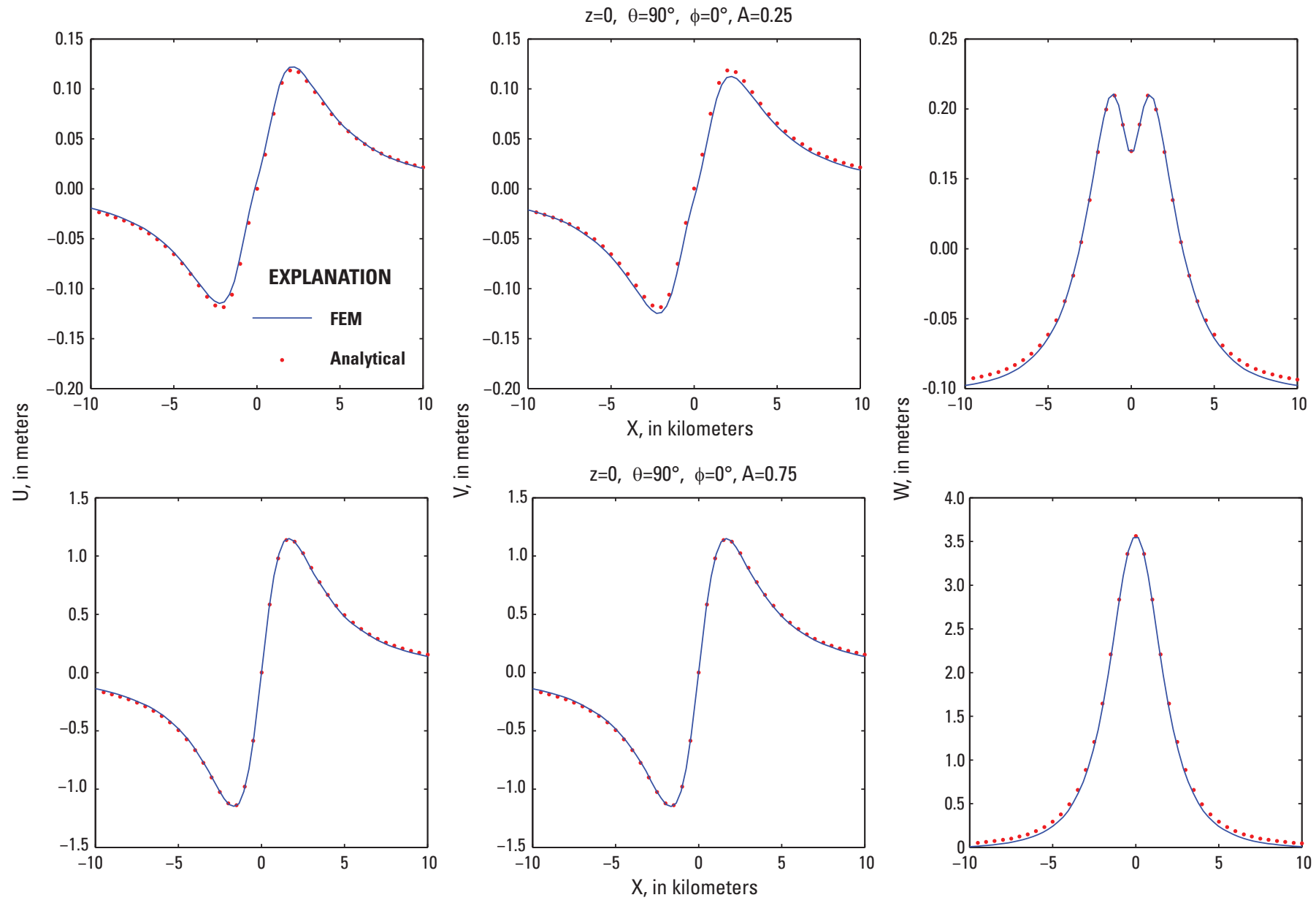

Figure 14. Free-surface deformation caused by the inflation of a vertical prolate spheroid $\left(\phi=0^{\circ}\right)$ for different values of the geometric aspect ratio $A$. Comparison between the three-dimensional semianalytical solution (analytical; equation 36), and a numerical finite element method model (FEM) of the same source. Source parameters are $x_{0}=y_{0}=0, z_{0}=3,000$ meters, length of semimajor axis $a=1000$ meters, $\Delta P / \mu=0.1, \mu=9.6$ Gigapascals and $v=0.25$. Because of a mathematical singularity at $\theta=90^{\circ}$, we used $\theta=89.99^{\circ}$. U: horizontal deformation (x or East component); $V$ : horizontal deformation (y or North component); W: vertical deformation (z or Up component). 

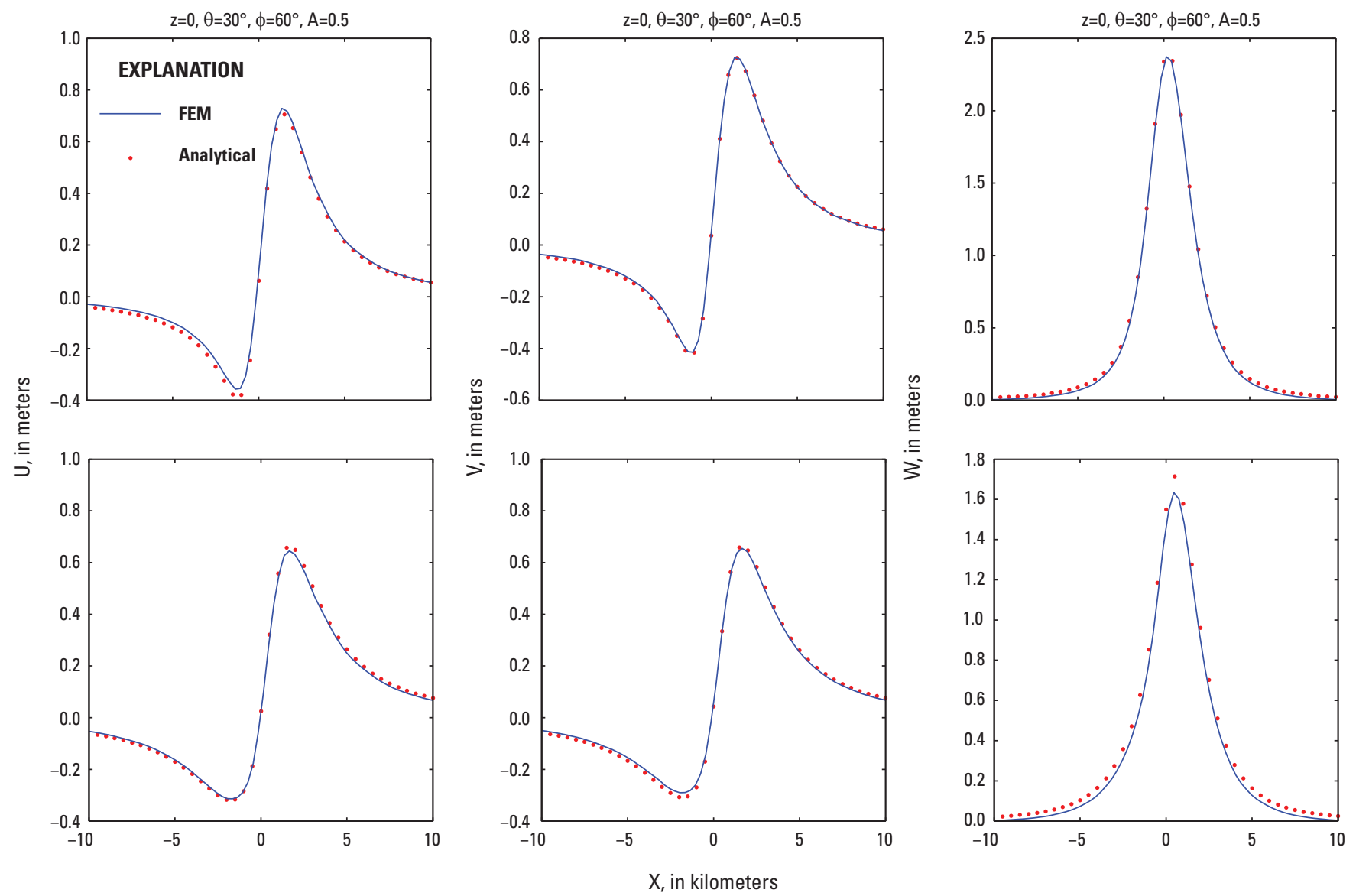

Figure 15. Free-surface deformation caused by the inflation of a tilted ( $\theta$ unequal to $0^{\circ}$ ) and rotated $\left(\phi\right.$ unequal to $\left.0^{\circ}\right)$ prolate spheroid. Comparison between the three-dimensional semianalytical solution (analytical; equation 36), and a numerical finite element method model (FEM) of the same source. Source parameters are $x_{0}=y_{0}=0, z_{0}=3,000$ meters, length of semimajor axis $a=1,000$ meters, $\Delta P / \mu=0.1, \mu=9.6$ Gigapascals and $v=0.25$. $U$ : horizontal deformation (x or East component); $V$ : horizontal deformation (y or North component); W: vertical deformation (z or Up component). See table 11 for numerical examples. 


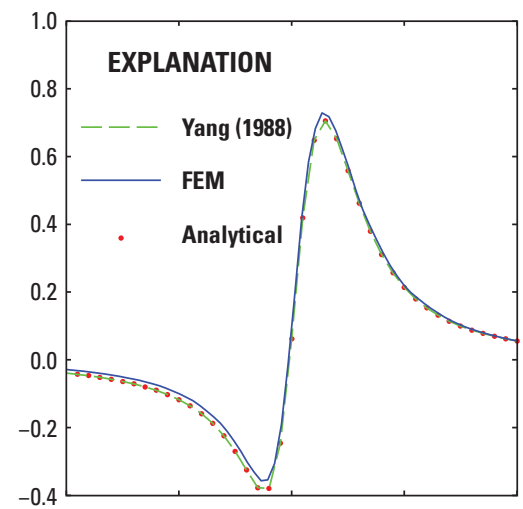

$z=0, \theta=30^{\circ}, \phi=60^{\circ}, A=0.5$
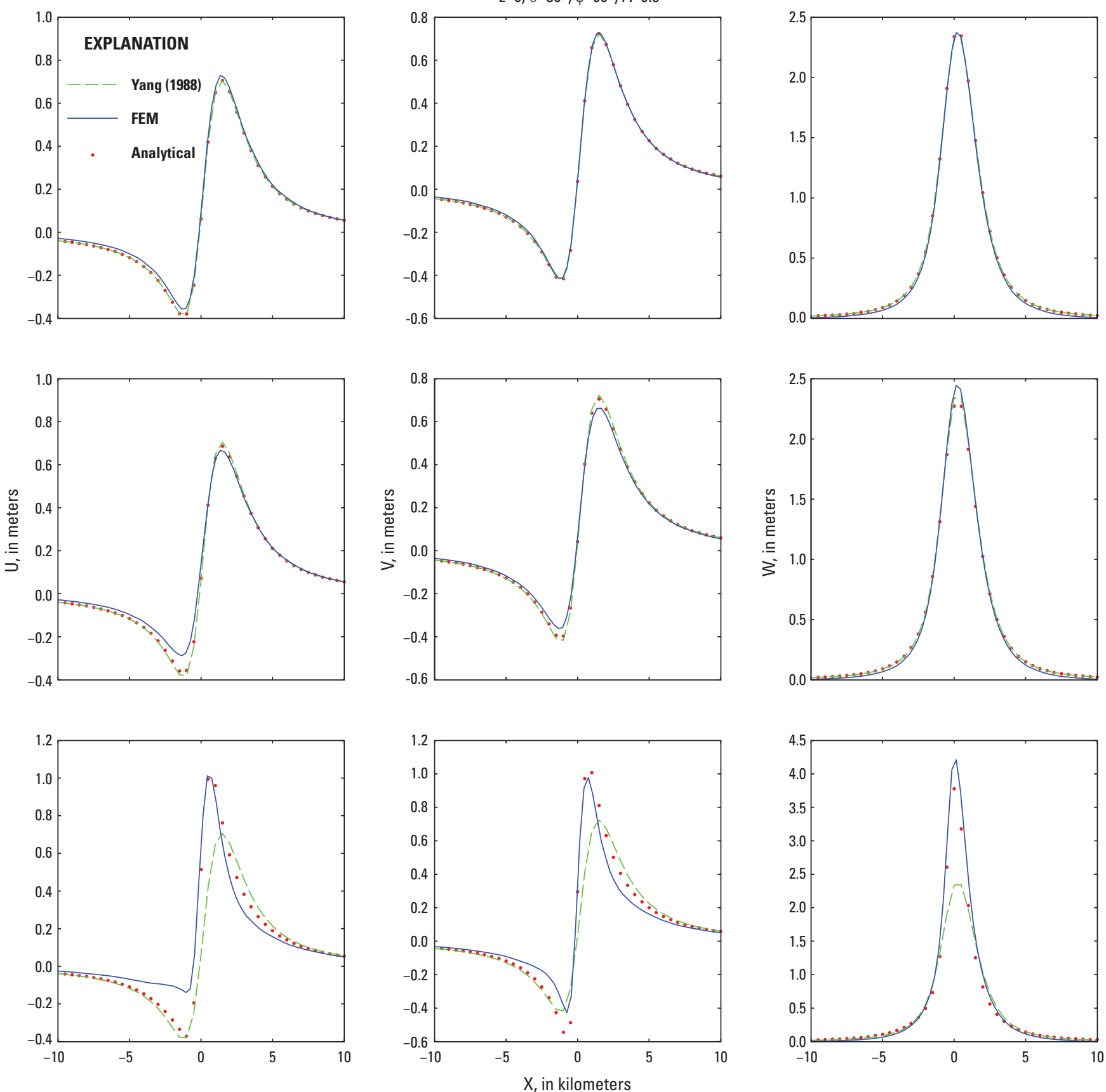

Figure 16. Internal deformation caused by the inflation of a tilted and rotated prolate spheroid. Comparison between the three-dimensional semianalytical solution described in this publication (analytical; equation 36), the original formulation by Yang and others (1988), and a numerical finite element method model (FEM) of the same source. Source parameters are $x_{0}=y_{0}=0, z_{0}=3,000$ meters, length of semimajor axis $a=1,000$ meters, $\Delta P / \mu=0.1, \mu=9.6$ Gigapascals and $v=0.25$. First row-displacements at the free surface; second row-displacements at shallow depths ( $z$ equals 150 meters); third row-displacements at greater depths ( $z$ equals 1,500 meters). $U$ : horizontal deformation (x or East component); $V$ : horizontal deformation (y or North component); $W$ : vertical deformation (z or Up component). See table 11 for numerical examples. 


\section{Volume Change}

The volume change of the ellipsoidal cavity is given by

$$
\Delta V=\frac{1-2 v}{2(1+v)} V \frac{\Delta P}{\mu}\left(\frac{p^{T}}{\Delta P}-3\right),
$$

where $V$ is the volume of the ellipsoidal cavity, and $p^{T}$ is the trace of the stress inside the ellipsoidal cavity (Amoruso and Crescentini, 2009). According to Amoruso and Crescentini (2009), the formula obtained by Tiampo and others (2000) for the volume change of an ellipsoid, $\Delta V=3 \Delta P V /(4 \mu)$, was derived by using a formula for strain which is valid for spheres only. As a consequence, Tiampo and others' (2000) expression is correct for spheres but incorrect for ellipsoids and always underestimates the volume change for an ellipsoid cavity.

The rigorous expression 51 can be approximated by the following empirical formulation for the volume change (fig. 17):

$$
\Delta V=V \frac{\Delta P}{\mu}\left(\frac{A^{2}}{3}-0.7 A+1.37\right) .
$$

The constants in equation 52 are determined by the polynomial best fitted to the numerical values from Amoruso and Crescentini (2009) and several FEM models (fig. 17).

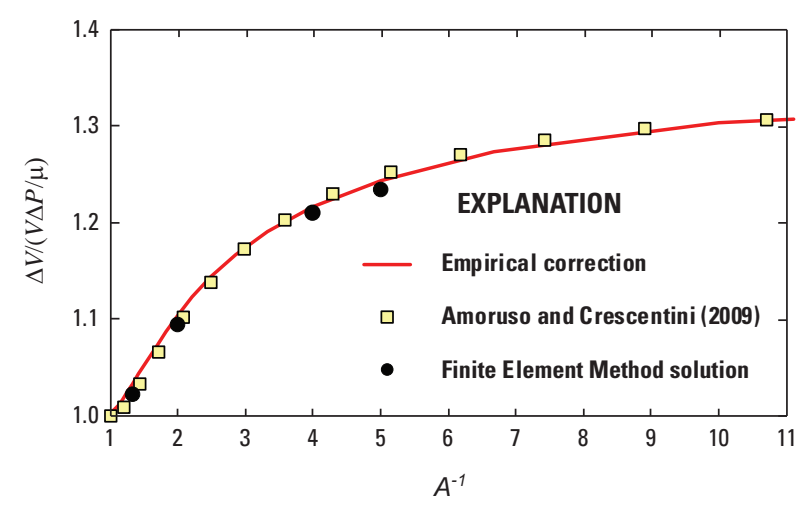

Figure 17. Verification of the empirical correction (equation 52) for the volume change of a pressurized prolate spheroid. $\Delta V /(V \Delta P / \mu)$ is the dimensionless volume change; $1 / A$, the reciprocal of the aspect ratio. 


\section{Ground Tilt}

Because of the complexity of Yang and others' (1988) expressions for the displacement, the ground tilt is computed by using finite-difference derivatives (see appendix 1). Figure 18 below shows the verification of ground-tilt calculations.
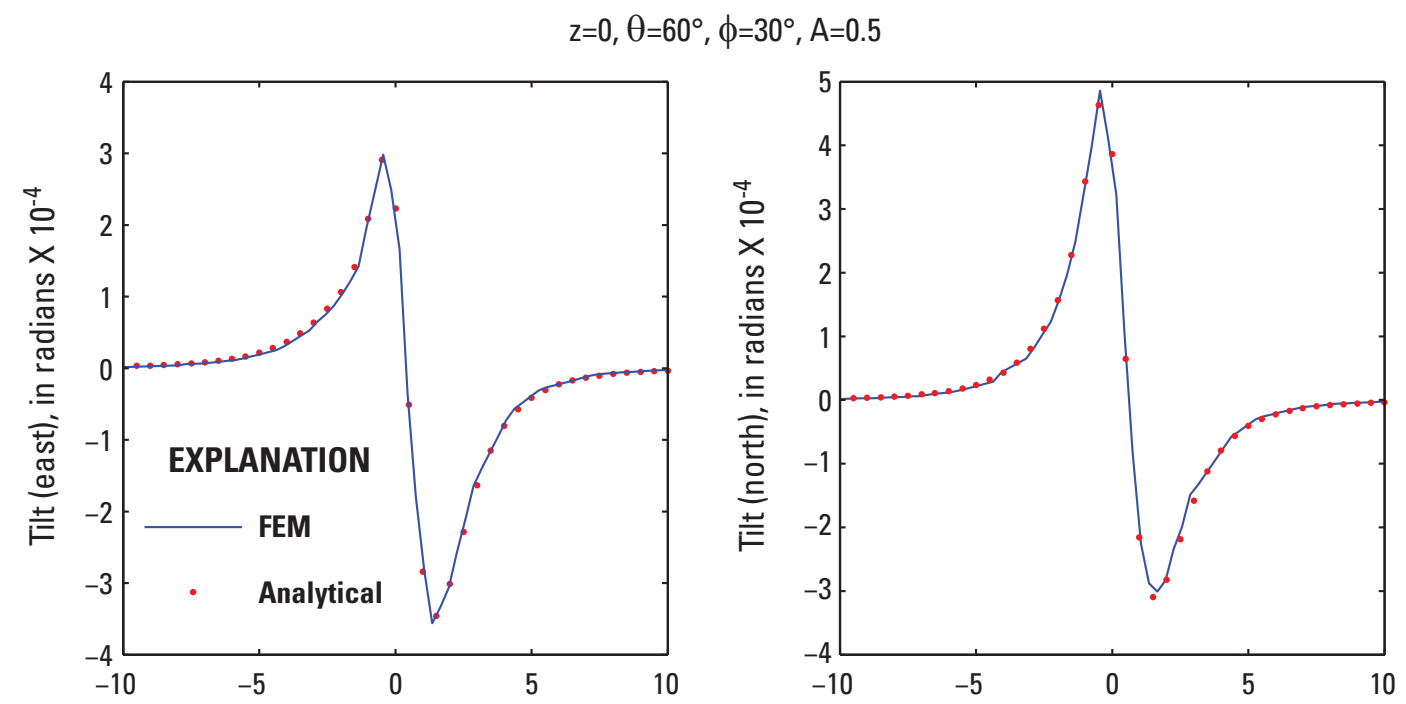

Figure 18. Ground tilt caused by the inflation of a tilted and rotated prolate spheroid. Comparison between the three-dimensional semianalytical solution described in this publication (analytical; equation 36 and appendix 1), and a numerical finite element method model (FEM) of the same source. Source parameters are $x_{0}=y_{0}=0, z_{0}=3,000$ meters, length of semimajor axis $a=1,000$ meters, $\Delta P / \mu=0.1, \mu=9.6$ Gigapascals and $\nu=0.25$. See table 11 for numerical examples. 


\section{Strain}

Finite-difference derivatives (see appendix 1) are used to compute the components of the displacement-gradient tensor (equation 32), verified in figure 19.
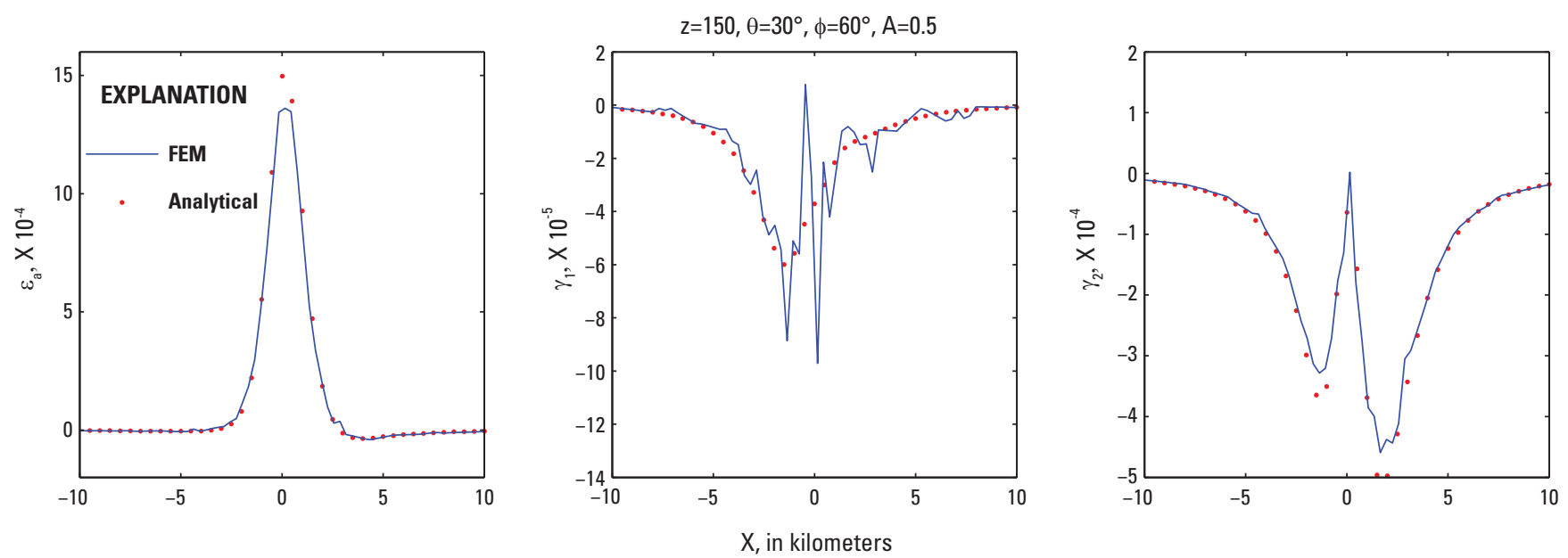

Figure 19. Internal strain caused by the inflation of a tilted and rotated prolate spheroid. Comparison between the threedimensional semianalytical solution described in this publication (analytical; equation 36 and appendix 1), and a numerical finite element method model (FEM) of the same source. $\varepsilon_{a}$ is the areal strain, $\gamma_{1}$ and $\gamma_{2}$ the shear strains. Source parameters are $x_{0}=y_{0}=0, z_{0}=3,000$ meters, length of semimajor axis $a=1,000$ meters, $\Delta P / \mu=0.1, \mu=9.6$ Gigapascals and $\nu=0.25$. See table 12 for numerical examples.

\section{Examples of Parameters Calculated for a Prolate Spheroid}

Table 11. Surface deformation and ground tilt.

[See equation 36 and appendix 1. Parameters are $x_{0}=0$ meters, $y_{0}=0$ meters, $z_{0}=3,000$ meters, $a=1,000$ meters, $A=0.5, \Delta P / \mu=0.1, \mu=9.6$ GigapascalPa, $v=0.25, \theta=30^{\circ}, \phi=60^{\circ} ; \mathrm{x}$ and $\mathrm{y}$, local Cartesian coordinates; $u_{x}, u_{y}$ and $u_{z}$, displacements at the free surface $\partial u_{z} / \partial x$ and $\partial u_{z} / \partial y$, tilt components]

\begin{tabular}{|c|c|c|c|c|c|c|}
\hline $\begin{array}{c}x \\
\left(10^{3} \text { meters }\right)\end{array}$ & $\begin{array}{c}y \\
\left(10^{3} \text { meters }\right)\end{array}$ & $\begin{array}{c}\mathbf{u}_{x} \\
\text { (meters) }\end{array}$ & $\underset{\text { (meters) }}{\mathbf{u}_{y}}$ & $\begin{array}{c}\mathbf{u}_{z} \\
\text { (meters) }\end{array}$ & $\begin{array}{l}\mathrm{du} / \mathrm{dx} \\
\left(\times 10^{-3}\right)\end{array}$ & $\begin{array}{l}\mathrm{du} / \mathrm{dy} \\
\left(\mathrm{X} \mathrm{10}^{-3}\right)\end{array}$ \\
\hline-4.0 & -4.0 & -0.1591 & -0.1736 & 0.1427 & 0.0368 & 0.0429 \\
\hline 2.0 & 2.0 & 0.6528 & 0.6737 & 1.0415 & -0.3015 & -0.2824 \\
\hline
\end{tabular}

Table 12. Internal deformation and strain.

[See equation 36 and appendix 1. Parameters are $x_{0}=0$ meters, $y_{0}=0$ meters, $z_{0}=3,000$ meters, $a=1,000$ meters, $A=0.5, \Delta P / \mu=0.1, \mu=9.6$ GigapascalPa, $v=0.25, \theta=30^{\circ}, \phi=60^{\circ}$; $\mathrm{x}$ and $\mathrm{y}$, local Cartesian coordinates; $u_{x}, u_{y}$ and $u_{z}$, internal deformation; $\varepsilon_{a}, \gamma_{1}$, and $\gamma_{2}$, strain]

\begin{tabular}{|c|c|c|c|c|c|c|c|c|}
\hline $\begin{array}{c}x \\
\left(10^{3} \text { meters }\right)\end{array}$ & $\begin{array}{c}y \\
\left(10^{3} \text { meters }\right)\end{array}$ & $\begin{array}{c}\mathrm{z} \\
\left(10^{3} \text { meters }\right)\end{array}$ & $\begin{array}{c}\mathbf{u}_{x} \\
\text { (meters) }\end{array}$ & $\stackrel{\mathbf{u}_{y}}{\text { (meters) }}$ & $\begin{array}{c}\mathbf{u}_{z} \\
\text { (meters) }\end{array}$ & $\begin{array}{c}\mathbf{e}_{a} \\
\left(\times 10^{-3}\right)\end{array}$ & $\begin{array}{c}\gamma_{1} \\
\left(\times 10^{-3}\right)\end{array}$ & $\begin{array}{c}\gamma_{2} \\
\left(X^{10-3}\right)\end{array}$ \\
\hline-4.0 & -4.0 & 1.5 & -0.1464 & -0.1597 & 0.1652 & -0.0041 & -0.0163 & -0.0922 \\
\hline 0.0 & 0.0 & 1.5 & 0.5138 & 0.2966 & 3.7764 & 4.1151 & -0.4081 & -0.7067 \\
\hline 2.0 & 2.0 & 1.5 & 0.5923 & 0.6309 & 0.8141 & 0.0412 & -0.0226 & -0.6365 \\
\hline
\end{tabular}




\section{Sill-Like Source}

A simple 3-D model of a sill intrusion is a horizontal penny-shaped crack in a semiinfinite elastic body (Fialko and others, 2001). The analytical expressions of Fialko and others (2001) are appropriate for a sill-like source with a radius as much as five times larger than its depth.

We consider a horizontal penny-shaped crack with radius $a$ and depth $z_{0}$ in an elastic halfspace (fig. 20A). The vertical axis in figure $20 \mathrm{~A}$ is the axis of symmetry (positive downwards), with an origin at the crack center. The surface of the half-space is assumed to be stress-free; $r$ and $z$ are spatial coordinates, and the index in parentheses corresponds to the upper and lower domains of the crack ( $i=1$ and 2 in fig. 20B). The boundary conditions for the problem are shown in figure 20A. Figure 21 shows the implementation of the model in the Matlab function fialko01.m

We translate and normalize all variables having dimensions of length with respect to the crack radius $a$ and all variables having dimensions of pressure with respect to the shear modulus $\mu$ of the elastic half-space:

$$
\begin{array}{ccc}
\bar{x}=\frac{x-x_{0}}{a}, & \bar{y}=\frac{y-y_{0}}{a}, & \bar{r}=\sqrt{\bar{x}^{2}+\bar{y}^{2}}, \\
\bar{z}=\frac{z-z_{0}}{a} & \text { and } & \bar{h}=\frac{z_{0}}{a}
\end{array}
$$

The general solution for the vertical and horizontal deformation of a half-space is given by equations 12 and 13 of Fialko and others (2001):

$$
\begin{aligned}
& U_{z}^{(1)}=\int_{0}^{\infty} {[((1-2 v) B-\xi(\bar{z}+\bar{h}) A) \sinh (\xi(\bar{z}+\bar{h}))} \\
&+(2(1-v) A-\xi(\bar{z}+\bar{h}) B) \cosh (\xi(\bar{z}+\bar{h}))] J_{0}(\xi \bar{r}) \mathrm{d} \xi \\
& U_{r}^{(1)}=\int_{0}^{\infty}[((1-2 v) A+\xi(\bar{z}+\bar{h}) B) \sinh (\xi(\bar{z}+\bar{h})) \\
&+ \\
&+(2(1-v) B+\xi(\bar{z}+h) A) \cosh (\xi(\bar{z}+h))] J_{1}(\xi \bar{r}) \mathrm{d} \xi
\end{aligned}
$$

All of the variables in equation 54 above are nondimensional, unless noted otherwise. The original equations 12 and 13 of Fialko and others (2001) have been changed in equation 54 as follows:

3. $2 U_{z}^{(1)}$ and $2 U_{r}^{(1)}$ must be replaced by $U_{z}^{(1)}$ and $U_{r}^{(1)}$, respectively, and

4. $d \xi / \sinh (\xi \bar{h})$ must be replaced by $d \xi$.

The functions $A$ and $B$ in equation 54 can be defined through the functions $\Phi$ and $\Psi$ as follows:

$$
\begin{aligned}
& A=\mathrm{e}^{-\xi \bar{h}}(\xi \bar{h} \Psi+(1+\xi \bar{h}) \Phi) . \\
& B=\mathrm{e}^{-\xi \bar{h}}((1-\xi \bar{h}) \Psi-\xi \bar{h} \Phi)
\end{aligned}
$$


The expression $\left(1-\mathrm{e}^{-2 \xi \bar{h}}\right)$ in the original equation 24 of Fialko and others (2001) should be replaced by $\mathrm{e}^{-\xi \bar{h}}$. Finally, the functions $\Phi$ and $\Psi$ can be expressed in terms of finite Fourier transforms:

$$
\begin{aligned}
& \Phi(\xi)=\int_{0}^{1} \sin (\xi t) \phi(t) d t \\
& \Psi(\xi)=\int_{0}^{1}\left[\frac{\sin (\xi \mathrm{t})}{\xi \mathrm{t}}-\cos (\xi \mathrm{t})\right] \psi(t) d t
\end{aligned}
$$

The integrals in equation 56 can be solved numerically (for example, by using the GaussLegendre quadrature method; Press and others, 1992, section 4.5). For a hydrostatic pressure distribution inside a penny-shaped crack with the boundary conditions given in figure $20 \mathrm{~A}$, the functions $\phi$ and $\psi$ are the solutions of the following Fredholm equations of the second kind:

$$
\begin{aligned}
& \phi(t)=-\frac{2 t}{\pi}+\frac{2}{\pi} \int_{0}^{1}\left[T_{1}(t, \bar{r}) \phi(\bar{r})+T_{3}(t, \bar{r}) \psi(\bar{r})\right] d \bar{r} \\
& \psi(t)=\frac{2}{\pi} \int_{0}^{1}\left[T_{4}(t, \bar{r}) \phi(\bar{r})+T_{2}(t, \bar{r}) \psi(\bar{r})\right] d \bar{r}
\end{aligned}
$$

where

$$
\begin{aligned}
& T_{1}(t, r)=4 h^{3}\left[P_{1}(t-\bar{r})-P_{1}(t+\bar{r})\right] \\
& T_{2}(t, r)=\frac{h}{t r}\left[P_{2}(t-\bar{r})-P_{2}(t+\bar{r})\right]+h\left[P_{3}(t-\bar{r})+P_{3}(t+\bar{r})\right] \\
& T_{3}(t, r)=\frac{h^{2}}{r}\left\{P_{4}(t-\bar{r})-P_{4}(t+\bar{r})-2 r\left[(t-\bar{r}) P_{1}(t-\bar{r})+(t+\bar{r}) P_{1}(t+\bar{r})\right]\right\} \\
& T_{4}(t, r)=T_{3}^{T}
\end{aligned} .
$$

and

$$
\begin{aligned}
& P_{1}(s)=\frac{12 \bar{h}^{2}-s^{2}}{\left(4 \bar{h}^{2}+s^{2}\right)^{3}} \\
& P_{2}(s)=\log \left(4 \bar{h}^{2}+s^{2}\right)+\frac{8 \bar{h}^{4}+2 s^{2} \bar{h}^{2}-s^{4}}{\left(4 \bar{h}^{2}+s^{2}\right)^{2}} \\
& P_{3}(s)=2 \frac{8 \bar{h}^{4}-2 s^{2} \bar{h}^{2}+s^{4}}{\left(4 \bar{h}^{2}+s^{2}\right)^{3}} \\
& P_{4}(s)=\frac{4 \bar{h}^{2}-s^{2}}{\left(4 \bar{h}^{2}+s^{2}\right)^{2}}
\end{aligned}
$$

The Fredholm equations 57, 58, and 59 can be solved numerically by using the Nystrom routine with the N-point Gauss-Legendre rule (Press and others, 1992, section 18.1).

Finally, solutions of the dimensionless equations 54 give the following expressions for the deformation (fig. 22; see tables 13 and 14 for numerical examples):

$$
\begin{aligned}
& u_{x}=a \frac{\Delta P}{\mu} U_{r}^{(1)} \frac{\bar{x}}{r} ; \\
& u_{y}=a \frac{\Delta P}{\mu} U_{r}^{(1)} \frac{\bar{y}}{r} . \\
& u_{z}=-a \frac{\Delta P}{\mu} U_{z}^{(1)}
\end{aligned}
$$




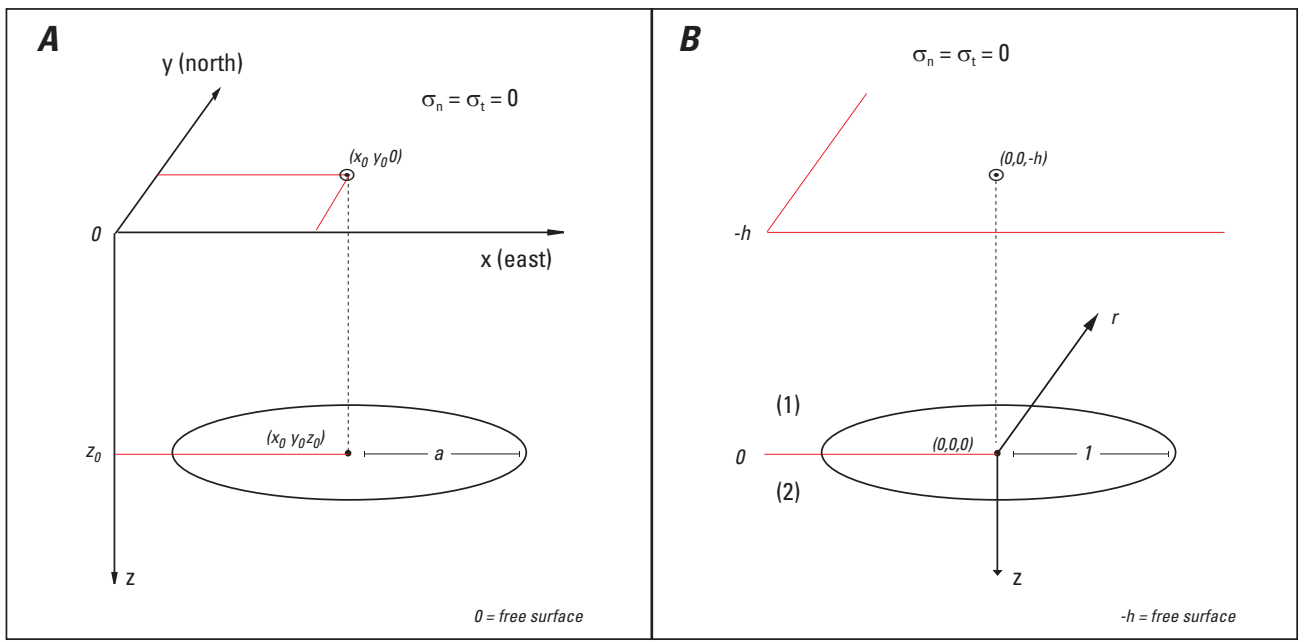

Figure 20. Definition of boundary conditions and geometry for a sill-like source (after Fialko and others, 2001). A, Dimensional coordinates and boundary-values. $x, y$, and $z$, local Cartesian coordinates; $a$, source radius; the normal stress $\sigma_{n}$ and shear stress $\sigma_{\tau}$ are equal to zero at the free surface; the source center is $\left(x_{0}, y_{0}, z_{0}\right)$, and the free surface is at $z=0$. B. Dimensionless coordinates and boundary-values. $r$ and $z$, local cylindrical coordinates; the source radius is equal to 1 ; the normal stress $\sigma_{n}$ and shear stress $\sigma_{\tau}$ are equal to zero at the free surface; the source center is at the origin of the coordinate system, and the free surface is at $z=-h$.

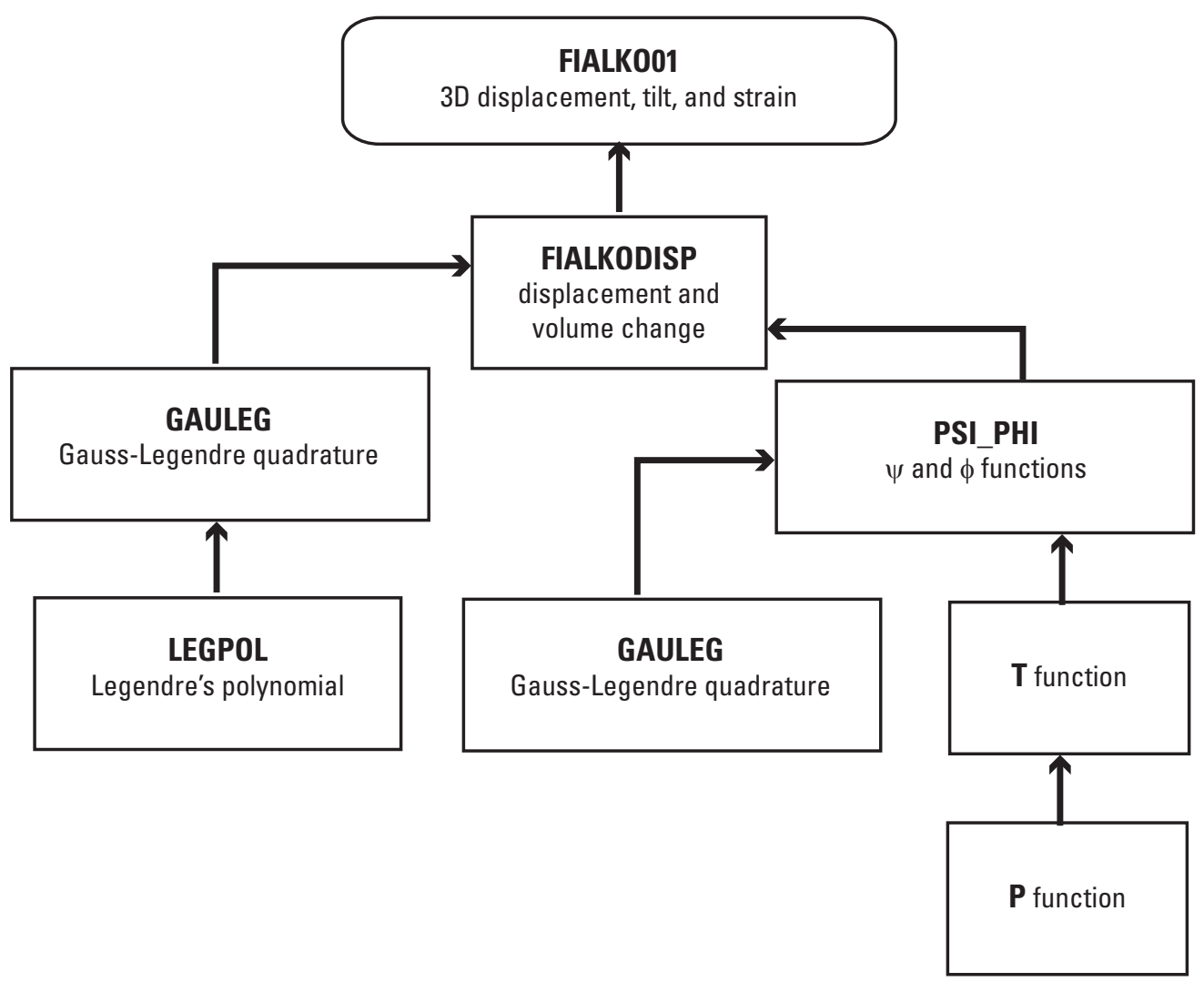

Figure 21. Matlab function fialk001.m flow chart for calculating and coding the parameters for a sill-like source. 

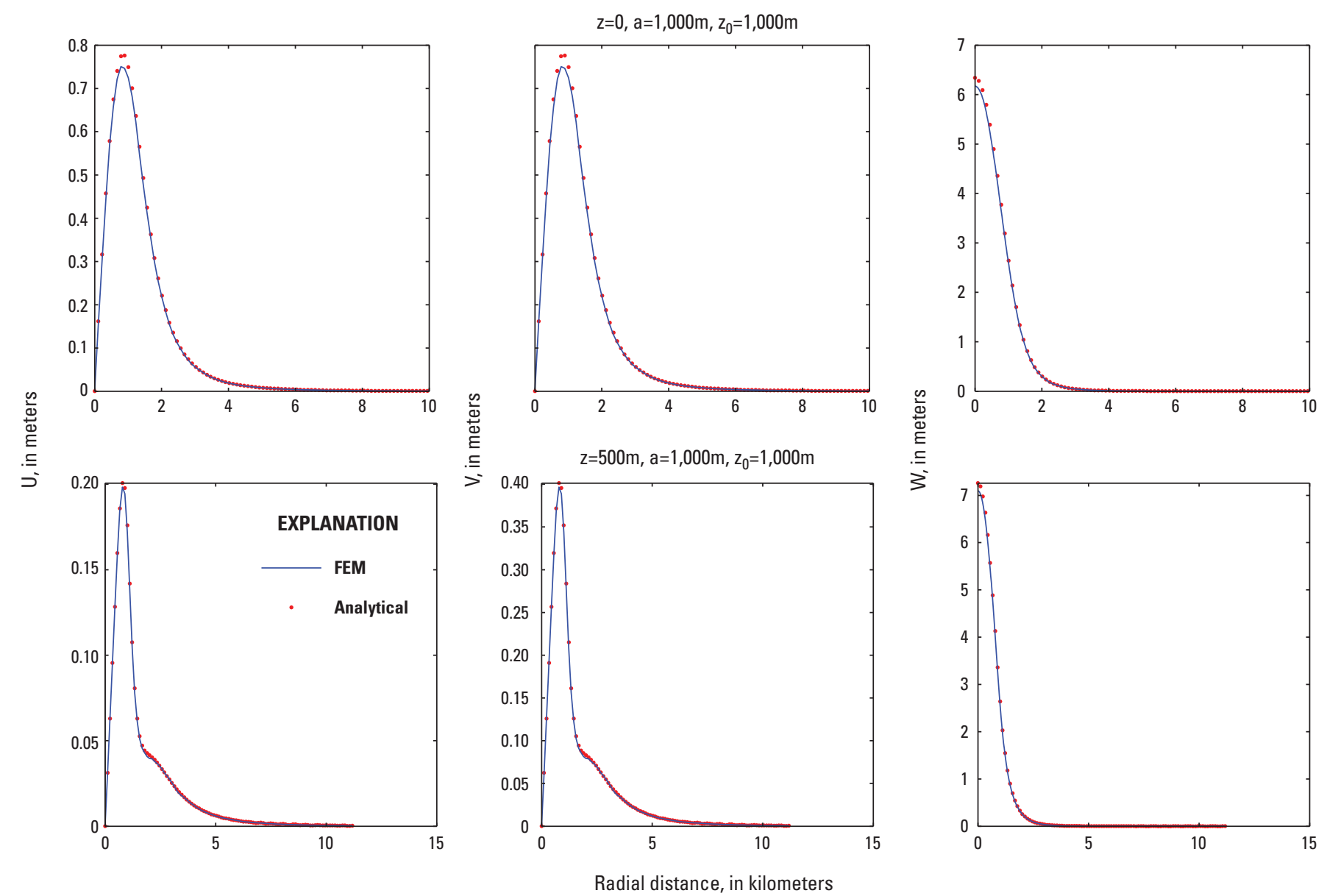

Figure 22. Graphs showing (top) surface deformation due to the inflation of a sill-like (or penny-crack) source; (bottom) internal deformation. Comparison between the three-dimensional semianalytical solution (analytical; equation 60), and a numerical finite element method model (FEM) of the same source. Source parameters are location $x_{0}=y_{0}=0$, depth $z_{0}=1,000$ meters, radius $a=1,000$ meters, $\Delta P / \mu=0.01, \mu=9.6$ Gigapascals, and $v=0.25 . U$ : horizontal deformation (x or East component); $V$ : horizontal deformation (y or North component); W: vertical deformation (z or Up component). See table 13 for numerical examples. 


\section{Volume Change}

For a uniformly pressurized crack, the expression for the volume change (fig. 23) is

$$
\Delta V=-4 \pi(1-v) \frac{\Delta P}{\mu} a^{3} \int_{0}^{1} t \phi(t) d t
$$

with $\phi(t)$ given by equation 57 .

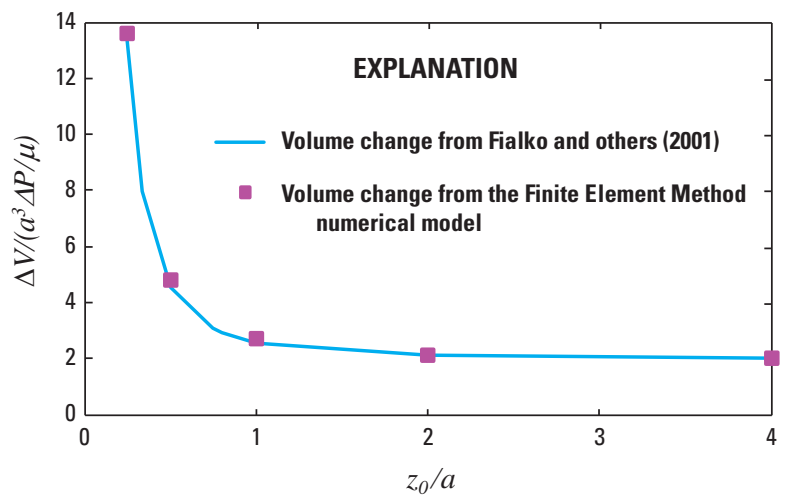

Figure 23. Volume change for a sill-like source (equation 61). $\Delta V /\left(a^{3} \Delta P / \mu\right), \Delta$ is the dimensionless volume change; $z_{0} / a$ the dimensionless source depth. The dimensionless pressure change is $\Delta P / \mu=0.01$.

\section{Ground Tilt}

Because of the complexity of Fialko and others' (2001) expressions for the displacement, the ground tilt is computed by using finite-difference derivatives (fig. 24, appendix 1).
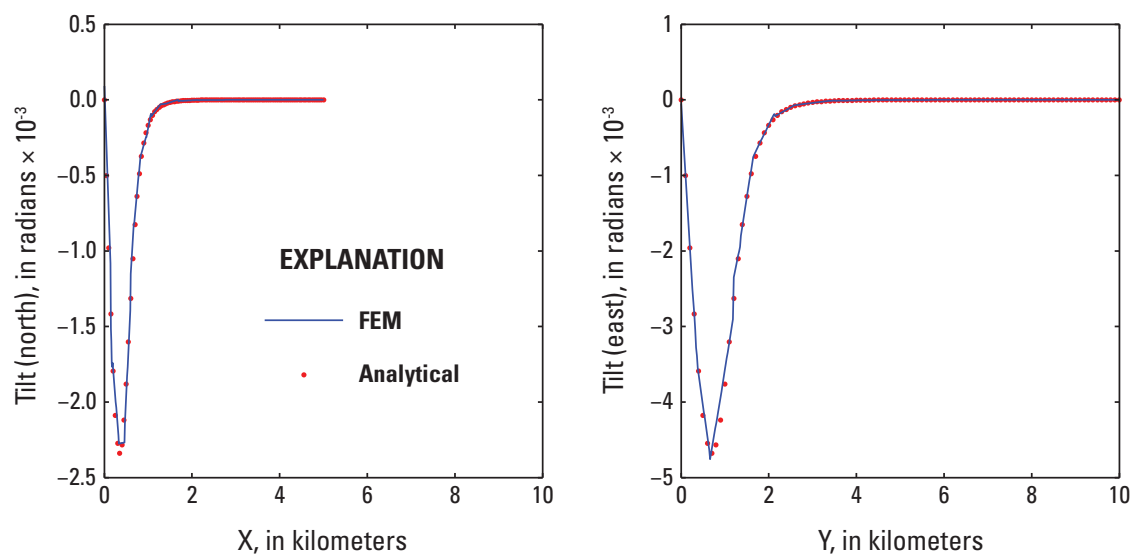

Figure 24. Ground tilt caused by the inflation of a sill-like source. Comparison between the three-dimensional semianalytical solution (analytical), and a numerical finite element method model (FEM) of the same source. Source parameters are location $x_{0}=y_{0}=0$, depth $z_{0}=1,000$ meters, radius $a=1,000$ meters, $\Delta P / \mu=0.01, \mu=9.6$ Gigapascals, and $\nu=0.25$. See table 13 for numerical examples. 


\section{Strain}

The components of the displacement gradient tensor are computed by finite-difference derivatives (fig. 25, appendix 1).
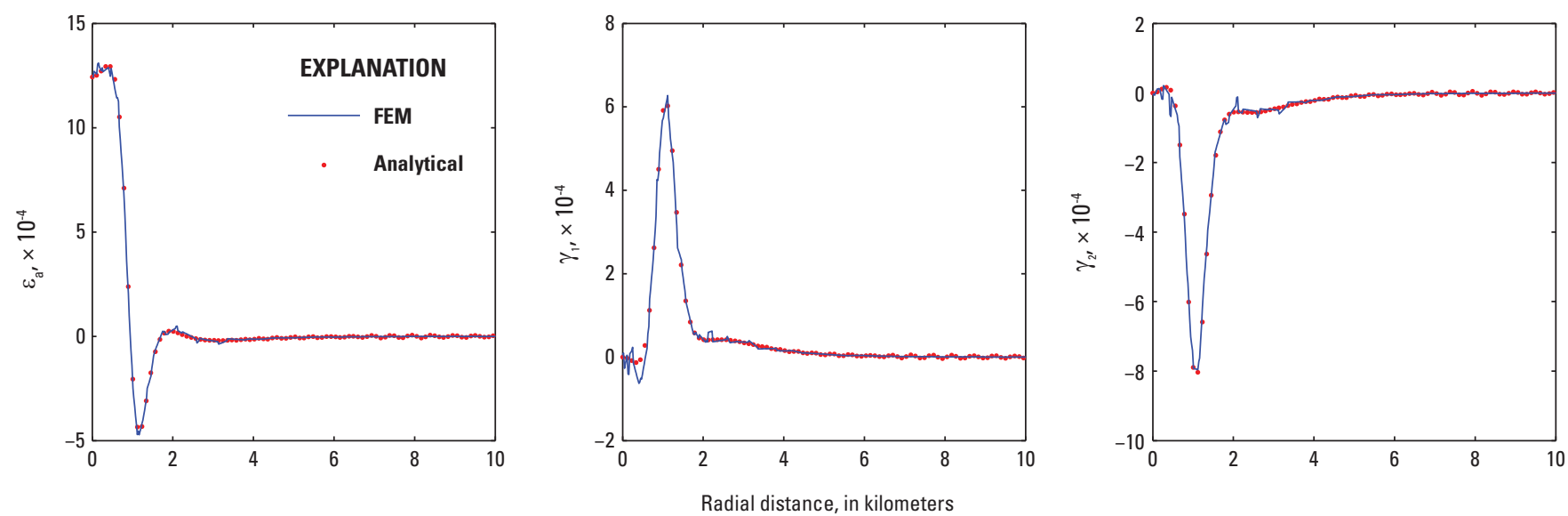

Figure 25. Internal strain caused by the inflation of a sill-like source. Comparison between the three-dimensional semianalytical solution described in this publication (analytical), and a numerical finite element method model (FEM) of the same source. $\varepsilon_{a}$ is the areal strain, $\gamma_{1}$ and $\gamma_{2}$ the shear strains. Source parameters are location $x_{0}=y_{0}=0$, depth $z_{0}=1,000$ meters, radius $a=1,000$ meters, $\Delta P / \mu=0.01, \mu=9.6$ Gigapascals. See table 14 for numerical examples.

\section{Examples of Parameters Calculated for a Sill-Like Structure}

Table 13. Surface deformation and tilt.

[See equation 22 and appendix 1. Parameters are $x_{0}=0$ meters, $y_{0}=0$ meters, $z_{0}=1,000$ meters, $a=1,000$ meters, $\Delta P / \mu=0.01, \mu=9.6 \mathrm{GPa}, v=0.25 ; \mathrm{r}$, radial distance from source center; $u_{x}, u_{y}$, and $u_{z}$, displacements at the free surface; $\partial u_{z} / \partial x$ and $\partial u_{z} / \partial y$, tilt components]

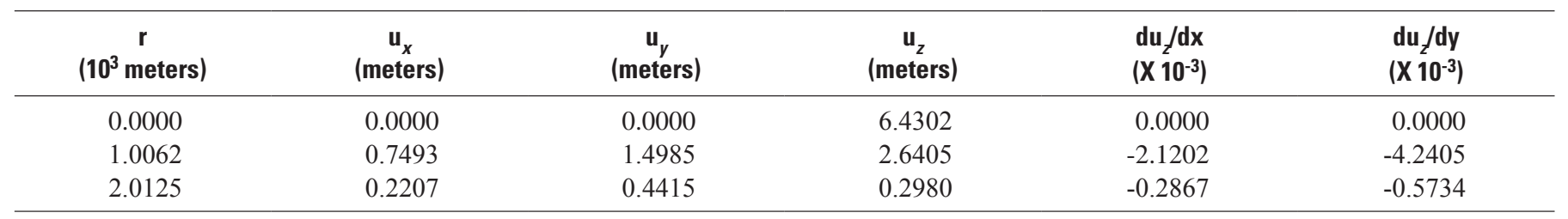

Table 14. Internal deformation and strain.

[See equation 22 and appendix 1. Parameters are $x_{0}=0$ meters, $y_{0}=0$ meters, $z_{0}=1,000$ meters, $a=1,000$ meters, $\Delta P / \mu=0.01, \mu=9.6$ GPa, $v=0.25 ; \mathrm{r}$, radial distance from source center; $u_{x}, u_{y}$, and $u_{z}$, and internal deformation; $\varepsilon_{a}, \gamma_{1}$ and $\gamma_{2}$, strain]

\begin{tabular}{|c|c|c|c|c|c|c|c|}
\hline $\begin{array}{c}r \\
\left(10^{3} \text { meters }\right)\end{array}$ & $\begin{array}{c}z \\
\left(10^{3} \text { meters }\right)\end{array}$ & $\begin{array}{c}u_{x} \\
\text { (meters) }\end{array}$ & $\begin{array}{c}u_{v} \\
\text { (meters) }\end{array}$ & $\underset{\text { (meters) }}{\mathbf{u}_{z}}$ & $\begin{array}{c}e_{a} \\
\left(\times 1^{-3}\right)\end{array}$ & $\begin{array}{c}\gamma_{1} \\
\left(\times 0^{-3}\right)\end{array}$ & $\begin{array}{c}\gamma_{2} \\
\left(\times 10^{-3}\right)\end{array}$ \\
\hline 0.0000 & 0.5000 & 0.0000 & 0.0000 & 7.2591 & 1.2431 & 0.0000 & 0.0000 \\
\hline 2.0125 & 0.5000 & 0.0415 & 0.0830 & 0.2585 & 0.0229 & 0.0416 & -0.0554 \\
\hline
\end{tabular}




\section{Surface Deformation and Ground Tilt for Rectangular Dikes and Faults}

The solutions proposed by Okada (1985) present a complete suite of closed analytical expressions (fig. 26) for the surface displacements and tilts caused by a strike-slip, dip-slip or tensile rectangular dislocation in a half-space that is homogenous, isotropic, flat, and elastic. These expressions are particularly compact and free from mathematical singularities

The rectangular source geometry is illustrated in the Cartesian coordinate system shown in figure 27A. The points $\left(x_{i}, y_{i}\right)$ and $\left(x_{f}, y_{f}\right)$ represent the initial and end coordinates of the source trace at the surface. The elementary dislocations $U$ correspond to the strike-slip $\left(U_{1}\right)$, dip-slip $\left(U_{2}\right)$, or tensile $\left(U_{3}\right)$ component of an arbitrary dislocation. In the convention used by Okada (1985), coordinates are assumed to be in kilometers, whereas $U$ and the displacements at the Earth's surface, $\mathbf{u}=\left[u_{x}, u_{y}, u_{z}\right]$, are in meters.

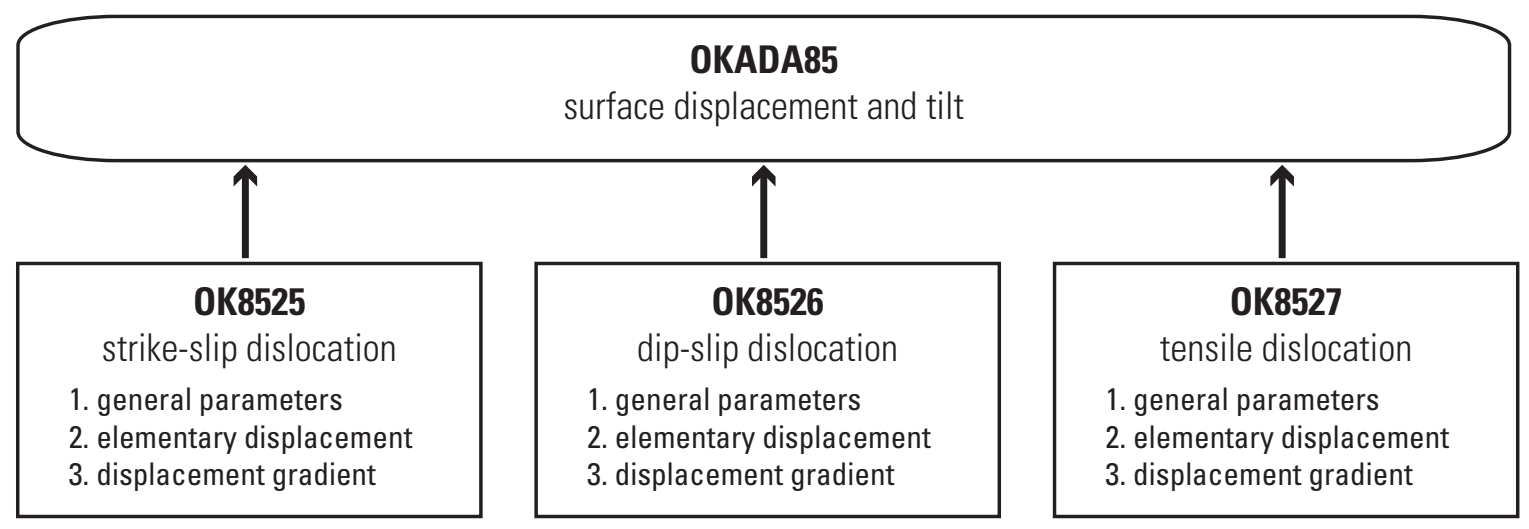

Figure 26. Program flow chart for rectangular dislocations (Okada, 1985).

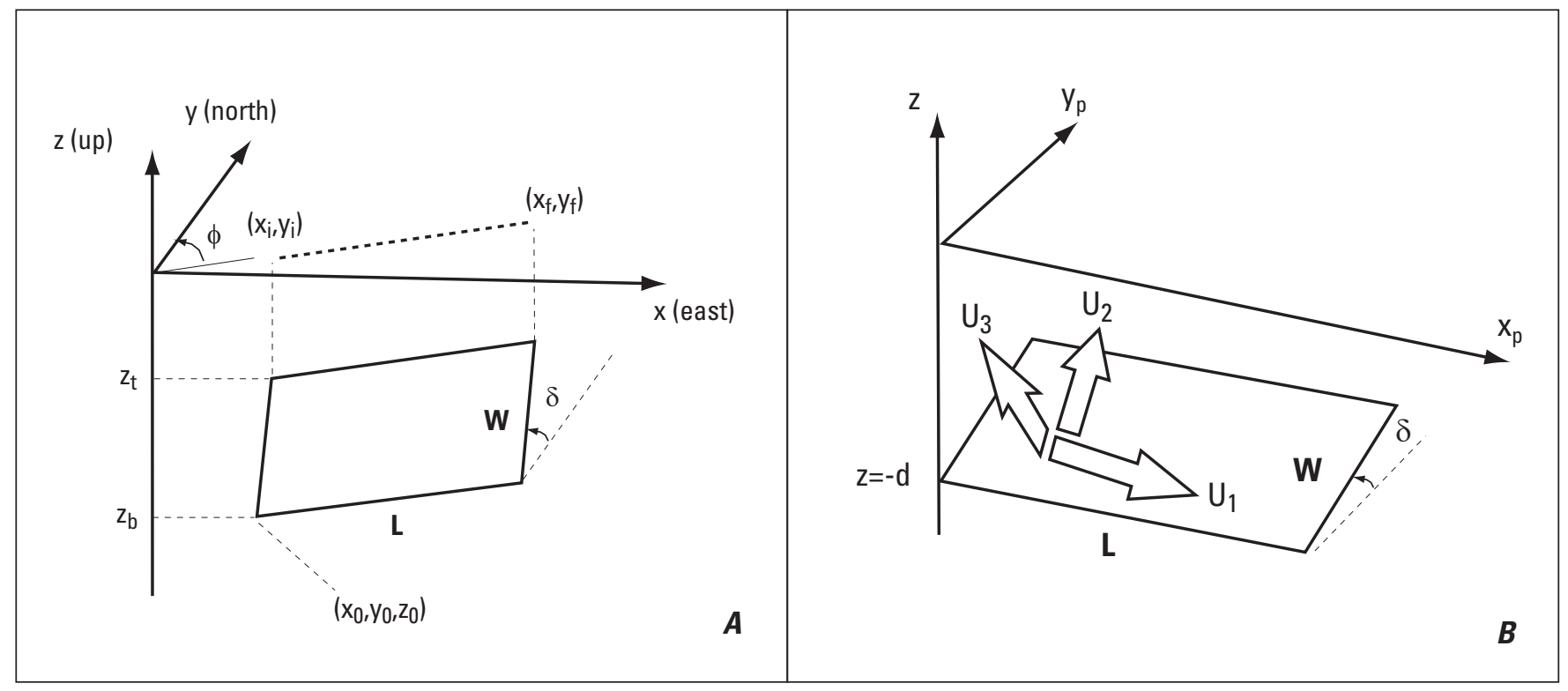

Figure 27. A, Geometry of the dislocation model in a Cartesian coordinate system; $B$, Geometry of the source model (after Okada, 1985). In Okada (1985), input coordinates are assumed to be in kilometers, whereas the dislocations and displacements are in meters. Strike-slip dislocation $U_{1}$ greater than 0 identifies a right lateral motion, dip-slip dislocation $U_{2}$ greater than 0 identifies a reverse motion, and tensile dislocation: $U_{3}$ greater than 0 identifies a tensile opening. 


\section{Displacement}

The angle $\phi$ is the strike of the fault

$$
\begin{aligned}
& p=y_{p} \cos \delta+d \sin \delta \\
& q=y_{p} \sin \delta-d \cos \delta
\end{aligned}
$$

and $\delta$ is the dip angle. The length $L$ and width $W$ of the rectangular dislocation are

$$
\begin{gathered}
L=\sqrt{\left(x_{f}-x_{i}\right)^{2}+\left(y_{f}-y_{i}\right)^{2}} \text { and } \\
W=\left|\frac{z_{b}-z_{t}}{\sin \delta}\right| .
\end{gathered}
$$

and the coordinates of the lower corner of the dislocation are (fig. 27A)

$$
\begin{aligned}
& x_{0}=x_{i}+\left(z_{b}-z_{t}\right) \cot \delta \cos \phi \\
& y_{0}=y_{i}-\left(z_{b}-z_{t}\right) \cot \delta \sin \phi \\
& z_{0}=z_{b}
\end{aligned} .
$$

Elementary expressions for surface deformation and the displacement-gradient matrix can be written as functions of nine sets of variables:

1. the fault type,

2. the coordinates of each dislocation corner (as given by $x_{p}, L$, and $W$ ),

3.

$$
x_{n}=x-x_{0} \quad y_{n}=y-y_{0} \quad z_{n}=-d=-z_{0},
$$

4.

$$
\begin{aligned}
& x_{p}=\sin \phi \cdot x_{n}+\cos \phi \cdot y_{n} \\
& y_{p}=-\cos \phi \cdot x_{n}+\sin \phi \cdot y_{n}
\end{aligned}
$$

5. the depth in kilometers of the lower left corner of the rectangular fault (d),

6. the Lame's elastic parameters $\mu$ and $\lambda$,

7. the dislocation $U$,

8. the dip angle $\delta$, and

9. the rotated coordinates $p$ and $q$

$$
\begin{aligned}
& p=y_{p} \cos \delta+d \sin \delta . \\
& q=y_{p} \sin \delta-d \cos \delta
\end{aligned}
$$

To compute the displacement, the function okada $85 . \mathrm{m}$ follows a three-step procedure: 
1. Compute the three components of the displacement and the six components of the displacement gradient matrix at the four corners of the dislocation:

$$
\begin{aligned}
& {\left[\begin{array}{lllllllll}
u & v & w & \frac{\partial u}{\partial x} & \frac{\partial u}{\partial y} & \frac{\partial v}{\partial x} & \frac{\partial v}{\partial y} & \frac{\partial w}{\partial x} & \left.\frac{\partial w}{\partial y}\right]_{1}=f_{i}\left(\xi, \eta, q, \mu, \lambda, U_{i}, \delta\right)=f_{i}\left(x_{p}, p, q, \mu, \lambda, U_{i}, \delta\right)
\end{array}\right.} \\
& {\left[\begin{array}{lllllllll}
u & v & w & \frac{\partial u}{\partial x} & \frac{\partial u}{\partial y} & \frac{\partial v}{\partial x} & \frac{\partial v}{\partial y} & \frac{\partial w}{\partial x} & \frac{\partial w}{\partial y}
\end{array}\right]_{2}=f_{i}\left(\xi, \eta, q, \mu, \lambda, U_{i}, \delta\right)=f_{i}\left(x_{p}, p-W, q, \mu, \lambda, U_{i}, \delta\right)}
\end{aligned}
$$

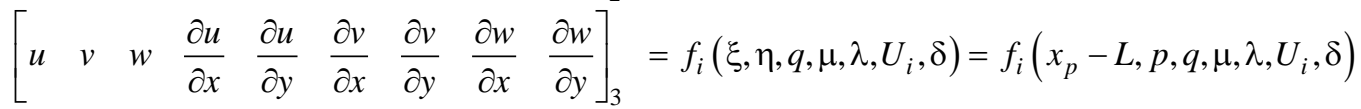

$$
\begin{aligned}
& {\left[\begin{array}{lllllllll}
u & v & w & \frac{\partial u}{\partial x} & \frac{\partial u}{\partial y} & \frac{\partial v}{\partial x} & \frac{\partial v}{\partial y} & \frac{\partial w}{\partial x} & \left.\frac{\partial w}{\partial y}\right]_{4} \\
= & =f_{i}\left(\xi, \eta, q, \mu, \lambda, U_{i}, \delta\right)=f_{i}\left(x_{p}-L, p-W, q, \mu, \lambda, U_{i}, \delta\right)
\end{array}\right.}
\end{aligned}
$$

where

$$
\begin{array}{ll}
(u, v, w) & \text { is the displacement, }[\cdots]_{k} \text { identifies the corners of the dislocation }(k=1,2,3,4), \\
\text { the subscript } i & \text { refers to the three different types of dislocation (1=strike, } 2=\text { dip, } 3=\text { tensile), } \\
\xi \text { and } \eta & \text { are dummy variables of integration. }
\end{array}
$$

2. Combine the displacements and displacement gradients from step 1 to compute the deformation, equation 69 , and tilt, equation 70, in Okada's (1985) reference system (fig. 27B).

$$
\begin{aligned}
& U_{x}^{p}=[u]_{1}-[u]_{2}-[u]_{3}+[u]_{4} \\
& U_{y}^{p}=[v]_{1}-[v]_{2}-[v]_{3}+[v]_{4}, \\
& U_{z}^{p}=[w]_{1}-[w]_{2}-[w]_{3}+[w]_{4}
\end{aligned}
$$

and

$$
\begin{aligned}
& \frac{\partial U_{z}^{p}}{\partial x}=\frac{\partial[w]_{1}}{\partial x}-\frac{\partial[w]_{2}}{\partial x}-\frac{\partial[w]_{3}}{\partial x}+\frac{\partial[w]_{4}}{\partial x} \\
& \frac{\partial U_{z}^{p}}{\partial y}=\frac{\partial[w]_{1}}{\partial y}-\frac{\partial[w]_{2}}{\partial y}-\frac{\partial[w]_{3}}{\partial y}+\frac{\partial[w]_{4}}{\partial y}
\end{aligned} .
$$

3. Rotate the deformation and tilts back to the original Cartesian reference system (fig. 27A):

and

$$
\begin{array}{ll}
\text { East } & u_{x}=\sin \phi \cdot U_{x}^{p}-\cos \phi \cdot U_{y}^{p} \\
\text { North } & u_{y}=\sin \phi \cdot U_{y}^{p}+\cos \phi \cdot U_{x}^{p}, \\
\text { Up } & u_{z}=U_{z}^{p}
\end{array}
$$

$$
\begin{array}{ll}
\text { tilt (East) } \frac{\partial u_{z}}{\partial x}=0.001\left(\frac{\partial U_{z}^{p}}{\partial x} \sin \phi-\frac{\partial U_{z}^{p}}{\partial y} \cos \phi\right) \\
\text { tilt (North) } \frac{\partial u_{z}}{\partial y}=0.001\left(\frac{\partial U_{z}^{p}}{\partial y} \sin \phi+\frac{\partial U_{z}^{p}}{\partial x} \cos \phi\right)
\end{array}
$$
meters. 


\section{Parameters for Displacements and Displacement Gradients Functions $f_{i}$}

The parameters and variables, which are listed below in the order they are coded, are the same for all the functions $f_{i}$.

1. Elastic parameter

$$
\alpha=\frac{\mu}{\lambda+\mu}=1-2 v
$$

2. Geometric transformations, equation 30 from Okada (1985), where $\xi$ and $\eta$ are dummy integration variables whose upper and lower limits are defined in equation 68

$$
\begin{aligned}
\tilde{y} & =\eta \cos \delta+q \sin \delta \\
\tilde{d} & =\eta \sin \delta-q \cos \delta \\
R^{2} & =\xi^{2}+\tilde{y}^{2}+\tilde{d}^{2} \\
X^{2} & =\xi^{2}+q^{2}
\end{aligned}
$$

3. Equation 28 (Okada,1985)

$$
\begin{aligned}
& I_{4}=\alpha \frac{1}{\cos \delta}[\ln (R+\tilde{d})-\sin \delta \ln (R+\eta)] \\
& I_{5}=\alpha \frac{2}{\cos \delta} \tan ^{-1}\left(\frac{\eta(X+q \cos \delta)+X(R+X) \sin \delta}{\xi(R+X) \cos \delta}\right) \\
& I_{3}=\alpha\left(\frac{1}{\cos \delta} \frac{\tilde{y}}{R+\tilde{d}}-\ln (R+\eta)\right)+\tan \delta I_{4} \\
& I_{1}=\alpha\left(-\frac{1}{\cos \delta} \frac{\xi}{R+\tilde{d}}\right)-\tan \delta I_{5} \\
& I_{2}=\alpha(-\ln (R+\eta))-I_{3}
\end{aligned}
$$

when $\xi=0$ (Okada, 1985, singularity condition ii, p. 1148), $I_{5}=0$;

when $R+\eta=0$ (Okada, 1985, singularity condition iii, p. 1148), replace $\ln (R+\eta)$ with $-\ln (R-\eta)$ in equation 74 ;

if $\cos \delta=0$, use equation 29 (Okada,1985);

$$
\begin{aligned}
& I_{1}=-0.5 \alpha \frac{\xi q}{(R+\tilde{d})^{2}} \\
& I_{3}=0.5 \alpha\left[\frac{\eta}{R+\tilde{d}}+\frac{\tilde{y} q}{(R+\tilde{d})^{2}}-\ln (\mathrm{R}+\eta)\right] \\
& I_{4}=-\alpha \frac{q}{R+\tilde{d}} \\
& I_{5}=-\alpha \frac{\xi \sin \delta}{R+\tilde{d}}
\end{aligned}
$$

4. Equation 40 (Okada,1985) 


$$
\begin{aligned}
& K_{3}=\alpha \frac{1}{\cos \delta}\left[\frac{q}{R} \frac{1}{R+\eta}-\frac{\tilde{y}}{R(R+\tilde{d})}\right] \\
& K_{1}=\alpha \frac{\xi}{\cos \delta}\left[\frac{1}{R(R+\tilde{d})}-\frac{\sin \delta}{R} \frac{1}{R+\eta}\right] ; \\
& K_{2}=\alpha\left[-\frac{\sin \delta}{R}+\frac{q \cos \delta}{R} \frac{1}{R+\eta}\right]-K_{3}
\end{aligned}
$$

if $\cos \delta=0$, use equation 41 from Okada (1985):

$$
\begin{aligned}
& K_{1}=\frac{\alpha \xi q}{R(R+\tilde{d})^{2}} \\
& K_{3}=\alpha \frac{\sin \delta}{R+\tilde{d}}\left[\frac{\xi^{2}}{R(R+\tilde{d})}-1\right]
\end{aligned}
$$

5. Equation 34 (Okada 1985)

$$
\begin{aligned}
& J_{1}=\alpha \frac{1}{\cos \delta}\left[\frac{\xi^{2}}{R(R+\tilde{d})^{2}}-\frac{1}{R+\tilde{d}}\right]-\tan \delta K_{3} \\
& J_{2}=\alpha \frac{1}{\cos \delta} \frac{\xi \tilde{y}}{R(R+\tilde{d})^{2}}-\tan \delta K_{1} \\
& J_{3}=\alpha\left(-\frac{\xi}{R} \frac{1}{R+\eta}\right)-J_{2} \\
& J_{4}=\alpha\left(-\frac{\cos \delta}{R}-\frac{q \sin \delta}{R} \frac{1}{R+\eta}\right)-J_{1}
\end{aligned}
$$

if $\cos \delta=0$, use equation 35 from Okada,1985)

$$
\begin{aligned}
& J_{1}=0.5 \alpha \frac{q}{(R+\tilde{d})^{2}}\left(\frac{2 \xi^{2}}{R(R+\tilde{d})}-1\right) \\
& J_{2}=0.5 \alpha \frac{\xi \sin \delta}{(R+\tilde{d})^{2}}\left(\frac{2 q^{2}}{R(R+\tilde{d})}-1\right)
\end{aligned}
$$

6. Equation 36 (Okada,1985)

$$
A_{c}=\frac{2 R+\xi}{R^{3}(R+\xi)^{2}} \quad A_{n}=\frac{2 R+\eta}{R^{3}(R+\eta)^{2}}
$$

7. If $R+\eta=0$ (Okada, 1985, singularity condition iii, p. 1148), replace $\ln (R+\eta)$ with $-\ln (R-\eta)$ and set $\frac{1}{R+\eta}=0$. 


\section{Elementary Function $f_{1}$ for a Strike-Slip Fault}

Elementary Displacement (Okada, 1985, equation 25)

$$
\begin{aligned}
u & =-\frac{U_{1}}{2 \pi}\left[\frac{\xi q}{R} \frac{1}{R+\eta}+\tan ^{-1}\left(\frac{\xi \eta}{q R}\right)+I_{1} \sin \delta\right] \\
v & =-\frac{U_{1}}{2 \pi}\left(\frac{\tilde{y} q}{R} \frac{1}{R+\eta}+q \cos \delta \frac{1}{R+\eta}+I_{2} \sin \delta\right) \\
w & =-\frac{U_{1}}{2 \pi}\left(\frac{\tilde{d} q}{R} \frac{1}{R+\eta}+q \sin \delta \frac{1}{R+\eta}+I_{4} \sin \delta\right)
\end{aligned}
$$

if $q=0$ (Okada, 1985, singularity condition i, p. 1148), then

$$
u=-\frac{U_{1}}{2 \pi}\left[\frac{\xi q}{R} \frac{1}{R+\eta}+I_{1} \sin \delta\right]
$$

Displacement Gradient (Okada, 1985, equation 37)

$$
\begin{aligned}
& \frac{\partial w}{\partial x}=\frac{U_{1}}{2 \pi}\left[-\xi q^{2} A_{n} \cos \delta+\left(\frac{\xi q}{R^{3}}-K_{1}\right) \sin \delta\right] \\
& \frac{\partial w}{\partial y}=\frac{U_{1}}{2 \pi}\left[\frac{\tilde{d} q}{R^{3}} \cos \delta+\left(\xi^{2} q A_{n} \cos \delta-\frac{\sin \delta}{R}+\frac{\tilde{y} q}{R^{3}}-K_{2}\right) \sin \delta\right]
\end{aligned}
$$

\section{Elementary Function $f_{2}$ for a Dip-Slip Fault}

Elementary Displacement (Okada, 1985, equation 26)

$$
\begin{aligned}
& u=-\frac{U_{2}}{2 \pi}\left(\frac{q}{R}-I_{3} \sin \delta \cos \delta\right) \\
& v=-\frac{U_{2}}{2 \pi}\left(\frac{\tilde{y} q}{R} \frac{1}{R+\xi}+\cos \delta \tan ^{-1} \frac{\xi \eta}{q R}-I_{1} \sin \delta \cos \delta\right) \\
& w=-\frac{U_{2}}{2 \pi}\left(\frac{\tilde{d} q}{R} \frac{1}{R+\xi}+\sin \delta \tan ^{-1} \frac{\xi \eta}{q R}-I_{5} \sin \delta \cos \delta\right)
\end{aligned}
$$

if $q=0$ (Okada, 1985, singularity condition i, p. 1148), then

$$
\begin{aligned}
& v=-\frac{U_{2}}{2 \pi}\left(\frac{\tilde{y} q}{R} \frac{1}{R+\xi}-I_{1} \sin \delta \cos \delta\right) \\
& w=-\frac{U_{2}}{2 \pi}\left(\frac{\tilde{d} q}{R} \frac{1}{R+\xi}-I_{5} \sin \delta \cos \delta\right)
\end{aligned}
$$


Displacement Gradient (Okada, 1985, equation 38)

$$
\begin{aligned}
& \frac{\partial w}{\partial x}=\frac{U_{2}}{2 \pi}\left(\frac{\tilde{d} q}{R^{3}}+\frac{q \sin \delta}{R} \frac{1}{R+\eta}+K_{3} \sin \delta \cos \delta\right) \\
& \frac{\partial w}{\partial y}=\frac{U_{2}}{2 \pi}\left[\tilde{y} \tilde{d} q A_{c}-\left(\frac{2 \tilde{d}}{R} \frac{1}{R+\xi}+\frac{\xi \sin \delta}{R} \frac{1}{R+\eta}\right) \sin \delta+K_{1} \sin \delta \cos \delta\right]
\end{aligned}
$$

\section{Elementary Function $f_{3}$ for a Tensile Crack}

Elementary Displacement (Okada, 1985, equation 27)

$$
\begin{aligned}
& u=\frac{U_{3}}{2 \pi}\left(\frac{q^{2}}{R} \frac{1}{R+\eta}-I_{3} \sin ^{2} \delta\right) \\
& v=\frac{U_{3}}{2 \pi}\left[-\frac{\tilde{d} q}{R} \frac{1}{R+\xi}-\sin \delta\left(\frac{\xi q}{r} \frac{1}{R+\eta}-\tan ^{-1}\left(\frac{\xi \eta}{q r}\right)\right)-I_{1} \sin ^{2} \delta\right] \\
& w=\frac{U_{3}}{2 \pi}\left[\frac{\tilde{y} q}{R} \frac{1}{R+\xi}+\cos \delta\left(\frac{\xi q}{r} \frac{1}{R+\eta}-\tan ^{-1}\left(\frac{\xi \eta}{q r}\right)\right)-I_{5} \sin ^{2} \delta\right]
\end{aligned}
$$

if $q=0$ (Okada, 1985, singularity condition i, p. 1148), then

$$
\begin{aligned}
& v=\frac{U_{3}}{2 \pi}\left[-\frac{\tilde{d} q}{R} \frac{1}{R+\xi}-\sin \delta\left(\frac{\xi q}{r} \frac{1}{R+\eta}\right)-I_{1} \sin ^{2} \delta\right] \\
& w=\frac{U_{3}}{2 \pi}\left[\frac{\tilde{y} q}{R} \frac{1}{R+\xi}+\cos \delta\left(\frac{\xi q}{r} \frac{1}{R+\eta}\right)-I_{5} \sin ^{2} \delta\right]
\end{aligned}
$$

Displacement Gradient (Okada, 1985, equation 38)

$$
\begin{aligned}
& \frac{\partial w}{\partial x}=-\frac{U_{3}}{2 \pi}\left(\frac{q^{2} \sin \delta}{R^{3}}-q^{3} A_{n} \cos \delta+K_{3} \sin ^{2} \delta\right) \\
& \frac{\partial w}{\partial y}=-\frac{U_{3}}{2 \pi}\left[\tilde{y} \sin \delta+\tilde{d} \cos \delta q^{2} A_{c}+\xi q^{2} A_{n} \sin \delta \cos \delta-\left(\frac{2 q}{R} \frac{1}{R+\xi}-K_{1}\right) \sin ^{2} \delta\right]
\end{aligned}
$$




\section{Verification}

Matlab solutions (function okada85.m) based on the analytical expressions by Okada (1985) for strike-slip (fig. 28), dip-slip (fig. 29) and tensile (figs. 30 and 31) dislocations are compared to solutions from COULOMB $3.3^{3}$ and numerical FEM models.

\section{Strike-Slip Fault}
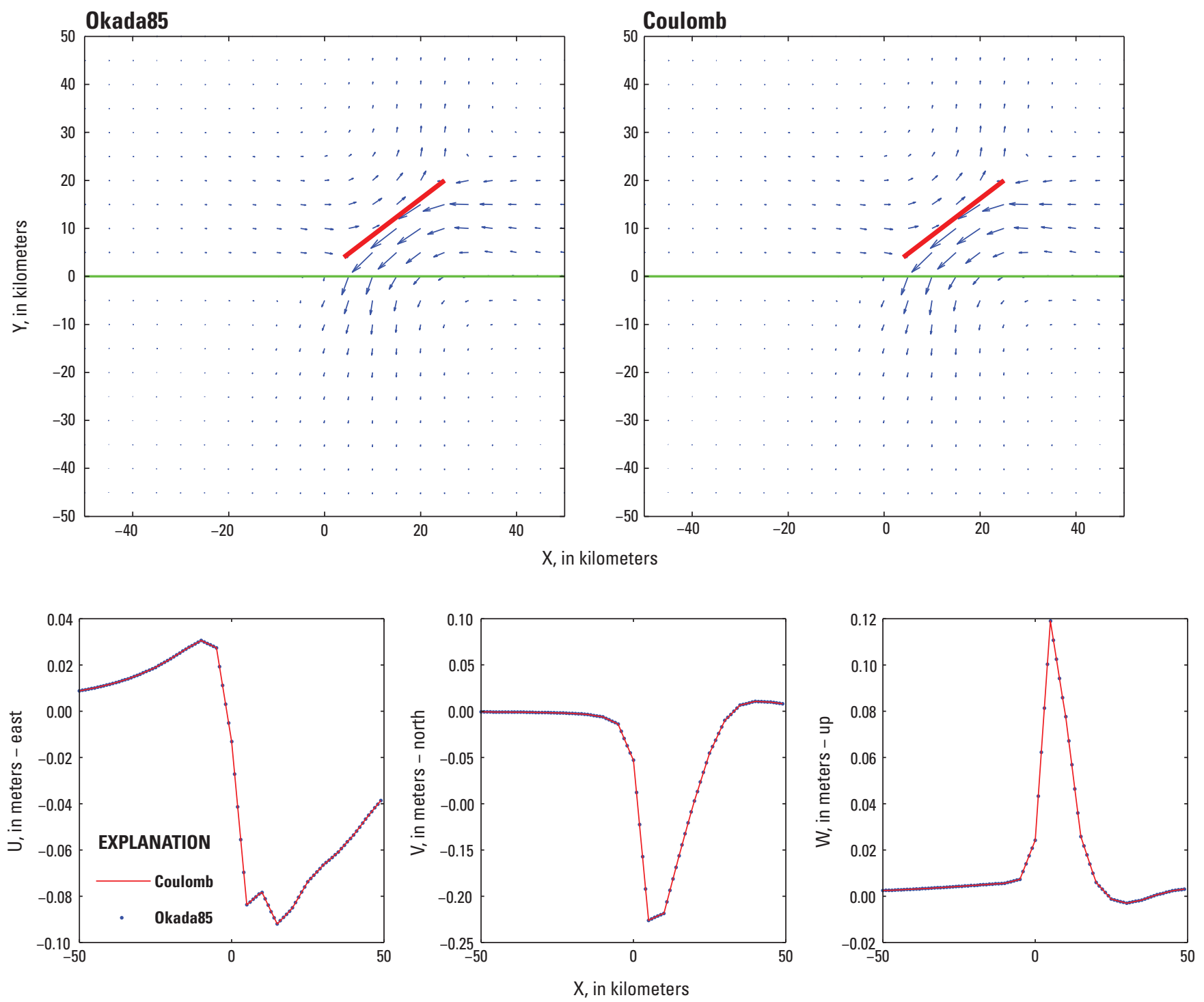

Figure 28. Vector (top) and profile plots (bottom) of the displacement field of a strike-slip fault (thick red line). The profile was taken along the green line. Okada85, Matlab script of Okada's (1985) expressions; Coulomb, solution from COULOMB 3.3. Fault parameters are $x_{i}=4,000$ meters, $y_{i}=4,000$ meters, $x_{f}=25,000$ meters, $y_{f}=20,000$ meters, $\delta=60^{\circ}, \mu=1$ Gigapascal, $v=0.25, U=1$ meter, $z_{t}=1000$ meters, and $z_{b}=10,000$ meters. See table 15 for numerical examples.

${ }^{3}$ COULOMB, a free software package developed by the USGS Earthquake Hazards Program, covers many of the same deformation sources as our Matlab codes, is based on Okada's (1992) equations (earthquake.usgs.gov/research/modeling/coulomb/overview.php), and is widely used. 


\section{Dip-Slip Fault}
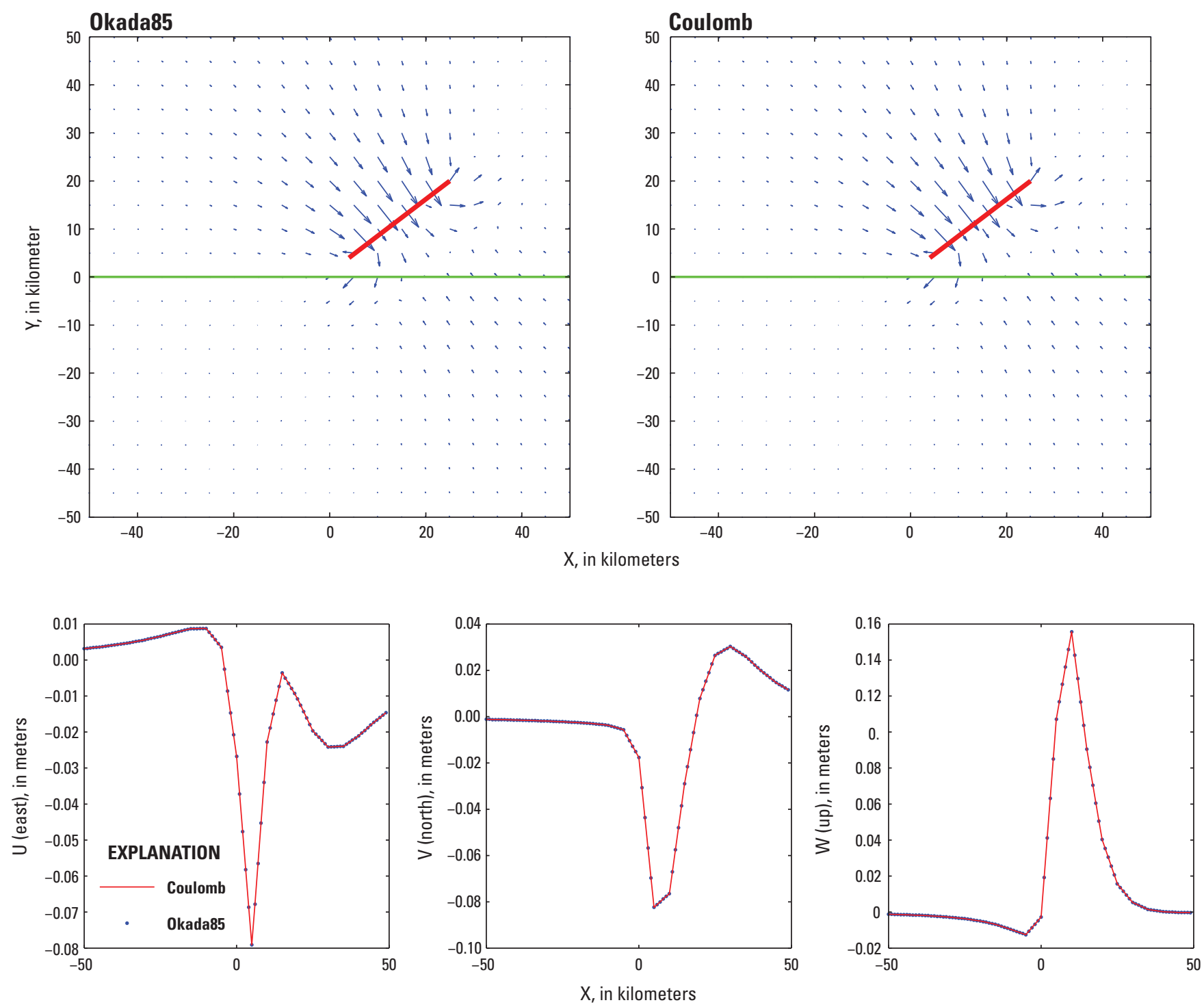

Figure 29. Vector (top) and profile plots (bottom) of the displacement field of a dip-slip fault (thick red line). The profile was taken along the green line. Okada85, Matlab script of Okada's (1985) expressions; Coulomb, solution from COULOMB 3.3. Fault parameters are: $x_{i}=4,000$ meters, $y_{i}=4,000$ meters, $x_{f}=25,000$ meters; $y_{f}=20,000$ meters; $\delta=60^{\circ} ; \mu=1 \mathrm{GPa} ; v=0.25 ; U=$ 1 meters; $z_{t}=1,000$ meters; $z_{b}=10,000$ meters. See table 16 for numerical examples. 


\section{Tensile Crack}
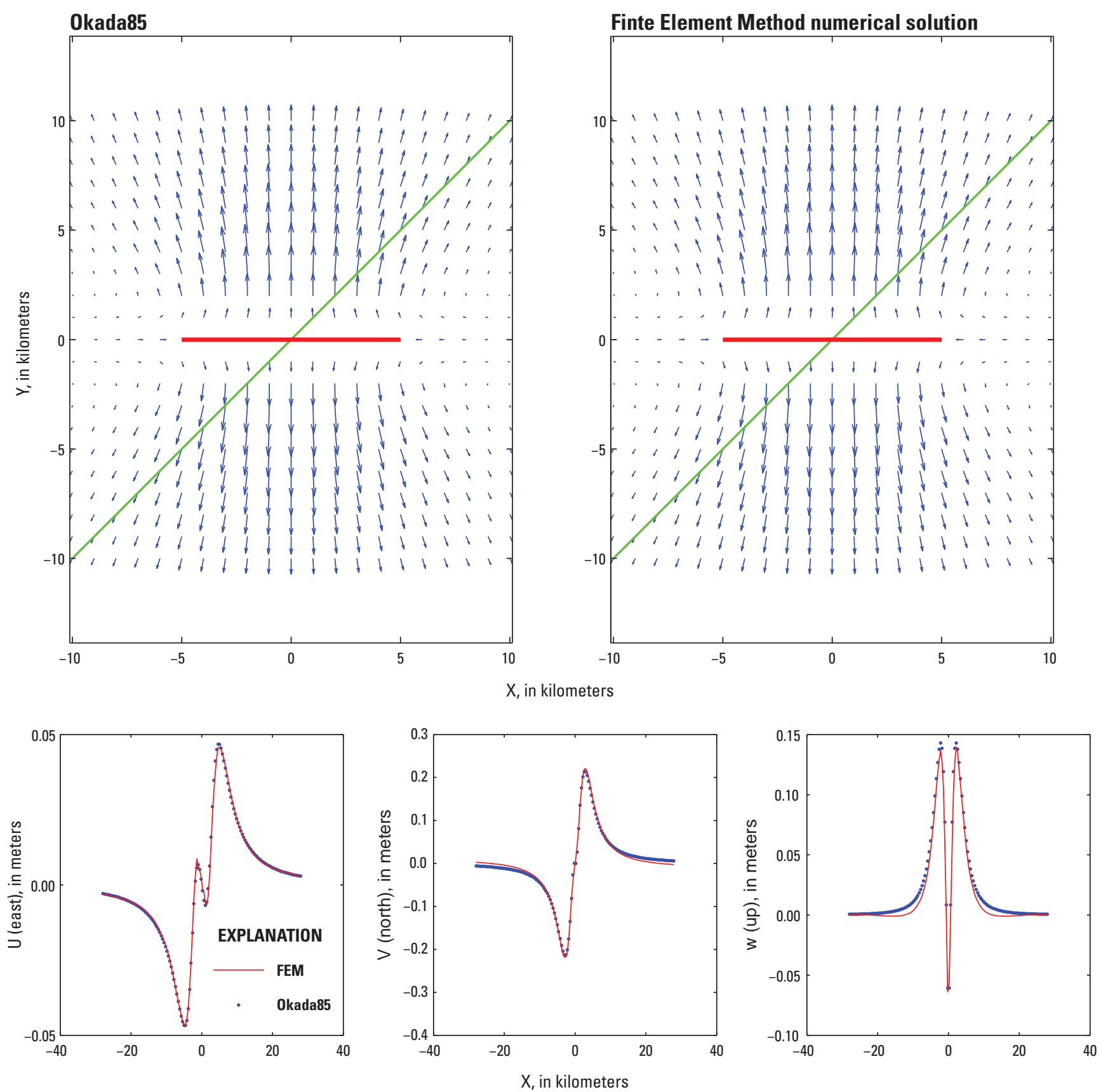

Figure 30. Vector (top) and profile plots (bottom) of the displacement field of a tensile crack (thick red line). The profile was taken along the green line. Okada85, Matlab script of Okada's (1985) expressions; FEM, numerical solution from FEM model. Fault parameters are: $x_{i}=-5,000$ meters; $y_{\mathrm{i}}=0$ meters, $x_{f}=5,000$ meters; $y_{f}=0$ meters; $\delta=90^{\circ} ; \mu=1 \mathrm{GPa} ; v=0.25 ; U=1$ meters; $z_{t}=1,000$ meters; $z_{b}=6,000$ meters. See table 17 for numerical examples. 


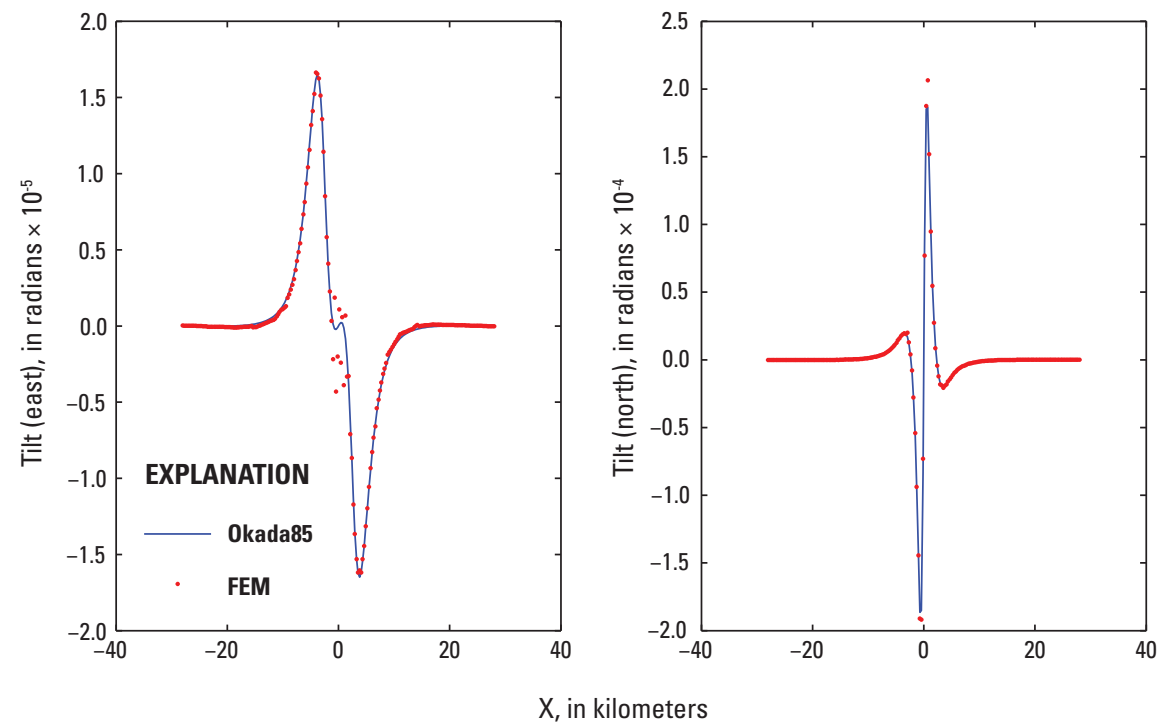

Figure 31. Profile plots showing tilt caused by a tensile crack. The profile was taken along the green line shown in figure 30. Okada85, Matlab script of Okada's (1985) expressions; FEM, numerical solution from FEM model. Fault parameters: $x_{\mathrm{i}}=-5,000$ meters, $y_{i}=0$ meters, $x_{f}=5,000$ meters; $y_{f}=0$ meters; $\delta=90^{\circ} ; \mu=1$ $\mathrm{GPa} ; v=0.25 ; U=1$ meters; $z_{t}=1,000$ meters; $z_{b}=6,000$ meters. See table 17 for numerical examples. 


\section{Examples of Surface Deformation and Ground Tilt for Three Types of Faults}

Table 15. Strike-slip fault.

[Vector and profile graphs shown in figure 28. Parameters are $x_{i}=4,000$ meters, $y_{i}=4,000$ meters, $x_{f}=25,000$ meters, $y_{f}=20,000$ meters, $\delta=60$ degrees, $\mu=1$ Gigapascal, $v=0.25, \mathrm{U}=1$ meter, $z_{t}=1,000$ meters, $z_{b}=10,000$ meters; $\mathrm{x}$ and $\mathrm{y}$, local coordinates; $u_{x}, u_{y}$ and $u_{z}$, displacements at the free surface; $\partial u_{z} / \partial x$ and $\partial u_{z} / \partial y$, tilt components]

\begin{tabular}{|c|c|c|c|c|c|c|}
\hline $\begin{array}{c}x \\
\left(10^{3} \text { meters }\right)\end{array}$ & $\begin{array}{c}y \\
\left(10^{3} \text { meters }\right)\end{array}$ & $\begin{array}{c}\boldsymbol{u}_{x} \\
\text { (meters) }\end{array}$ & $\begin{array}{c}u_{y} \\
\text { (meters) }\end{array}$ & $\begin{array}{c}u_{z} \\
\text { (meters) }\end{array}$ & $\begin{array}{r}d u_{z} / d x \\
\left(X 10^{-5}\right)\end{array}$ & $\begin{array}{l}d u / d y \\
\left(\times 10^{-5}\right)\end{array}$ \\
\hline-5.0000 & 0.0000 & 0.0274 & -0.0141 & 0.0073 & 0.0837 & -0.0250 \\
\hline 5.0000 & 0.0000 & -0.0837 & -0.2261 & 0.1190 & 1.3779 & 2.0268 \\
\hline
\end{tabular}

Table 16. Dip-slip fault.

[Vector and profile graphs shown in figure 29. Parameters are $x_{i}=4,000$ meters, $y_{i}=4,000$ meters, $x_{f}=25,000$ meters, $y_{f}=20,000$ meters, $\delta=60$ degrees, $\mu=$ 1 Gigapascal, $v=0.25, \mathrm{U}=1$ meter, $z_{t}=1,000$ meters, $z_{b}=10,000$ meters; $\mathrm{km}$, kilometers; $\mathrm{x}$ and $\mathrm{y}$, local coordinates; $u_{x}, u_{1}$ and $u_{z}$, displacements at the free surface; $\partial u_{z} / \partial x$ and $\partial u_{z} / \partial y$, tilt components]

\begin{tabular}{|c|c|c|c|c|c|c|}
\hline $\begin{array}{c}x \\
(\mathbf{k m})\end{array}$ & $\begin{array}{c}y \\
(10 \mathrm{~km})\end{array}$ & $\underset{\text { (meters) }}{\mathbf{u}_{\mathbf{x}}}$ & $\underset{\text { (meters) }}{u_{y}}$ & $\underset{\text { (meters) }}{\mathbf{u}_{\mathrm{z}}}$ & $\begin{array}{l}\mathrm{du}_{z} / \mathrm{dx} \\
\left(X^{\left.10^{-5}\right)}\right.\end{array}$ & $\begin{array}{l}\mathrm{du}_{z} / \mathrm{dy} \\
\left(\times 10^{-5}\right)\end{array}$ \\
\hline-5.0000 & 0.0000 & 0.0035 & -0.0057 & -0.0126 & -0.0321 & -0.1479 \\
\hline 5.0000 & 0.0000 & -0.0791 & -0.0824 & 0.1071 & 3.1341 & 3.0545 \\
\hline
\end{tabular}

Table 17. Tensile crack.

[Vector and profile graphs shown in figure 30; graphs of tilt profiles shown in figure 31. Parameters are $x_{i}=-5,000$ meters, $y_{i}=0$ meters, $x f=5,000$ meters, $y_{f}=0$ meters, $\delta=90$ degrees, $\mu=1$ Gigapascal, $v=0.25, \mathrm{U}=1$ meter, $z_{t}=1,000$ meters, $z_{b}=6,000$ meters; $\mathrm{x}$ and y, local coordinates; $u_{x}, u_{y}$ and $u_{z}$, displacements at the free surface; $\partial u_{z} / \partial x$ and $\partial u_{z} / \partial y$, tilt components]

\begin{tabular}{|c|c|c|c|c|c|c|}
\hline $\begin{array}{c}x \\
\left(10^{3} \text { meters }\right)\end{array}$ & $\begin{array}{c}y \\
\left(10^{3} \text { meters }\right)\end{array}$ & $\begin{array}{c}\boldsymbol{u}_{x} \\
\text { (meters) }\end{array}$ & $\begin{array}{c}u_{y} \\
\text { (meters) }\end{array}$ & $\begin{array}{c}\boldsymbol{u}_{z} \\
\text { (meters) }\end{array}$ & $\begin{array}{r}d u_{z} / d x \\
\left(X 10^{-5}\right)\end{array}$ & $\begin{array}{c}d u_{z} / d y \\
\left(X 10^{-5}\right)\end{array}$ \\
\hline 0.2010 & 0.2010 & -0.0019 & -0.0001 & -0.0609 & 0.0128 & 11.4199 \\
\hline 5.0251 & 5.0251 & 0.0467 & 0.1428 & 0.0618 & -1.2701 & -1.2241 \\
\hline
\end{tabular}




\section{Internal Deformation and Strain for Rectangular Dikes and Faults}

The solutions proposed in Okada (1992) present a complete suite of closed analytical expressions for the internal deformation and strain caused by a strike-slip, dip-slip, or tensile rectangular dislocation (fig. 32) in a half-space that is homogeneous, isotropic, flat, and elastic. These expressions are particularly compact and free from mathematical singularities.

\section{Source Geometry}

The rectangular source geometry and the Cartesian coordinate system for Okada (1992) are identical to those in the coding of Okada (1985, see section, "Surface Displacement and Tilt"; in this report, fig. 27, and equations 62-66).

\section{Displacement}

Elementary expressions for internal deformation and the displacement-gradient matrix can be written as functions of the fault type (fault), the coordinates of each fault corner (as given by $x_{p}, L$, and $W$ ), the depth in kilometers of the lower left corner of the rectangular fault $(c)$, the Lame's elastic parameters $\mu$ and $\lambda$, the dislocation $U$, the dip angle $\delta$, the rotated coordinate $y_{p}$, and the depth of the point of internal deformation $z$.

The displacement and displacement gradient matrix are given by the superposition of four different contributions (Okada, 1992, table 6):

1. infinite medium at depth $z$ (Okada, 1992, p. 1031) designated by $A$ in equation 91

2. the mirror image of $u_{A}$ at depth $-Z$ (Okada, 1992, p. 1031) designated by $A$ and $\sim$ in equation 92

3. the free-surface deformation (Okada, 1992, equation 18) designated by $B$ in equation 93

4. a depth-multiplied term (Okada, 1992, p. 1031) designated by $C$ in equation 94

To compute the displacement, follow a four-step procedure:

1. Compute the value of the elementary displacement for each contribution at each fault corner $a-d$.

2. Combine the values computed in step 1 for each contribution.

3. Compute the deformation in Okada's (1992) coordinate system (as shown in fig. 27B).

4. Rotate the deformation back to the Cartesian coordinate system (fig. 27A).

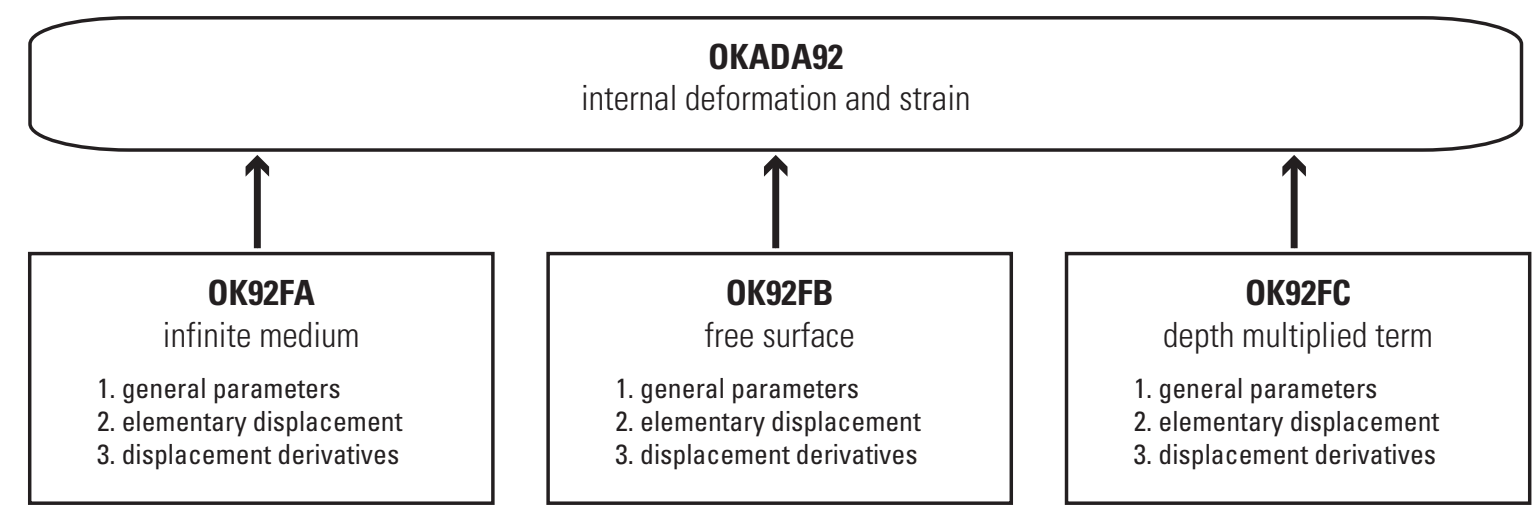

Figure 32. Program flow chart for rectangular dislocations (Okada, 1992). 


\section{Step 1-Elementary Displacement and Gradient}

In the equations below, $u$ is the displacement; $j$ the $x$-derivative and $k$ the $y$-derivative; and 1,2 , and 3 represent the strike-slip, dip-slip, and tensile components, respectively.

1. For an infinite medium at depth $z$ (equations for $f_{A}$ are in tables 18-21, p. 58-59),

$$
\begin{aligned}
& {\left[u_{1}^{A} u_{2}^{A} u_{3}^{A} j_{1}^{A} j_{2}^{A} j_{3}^{A} k_{1}^{A} k_{2}^{A} k_{3}^{A}\right]_{a}=f_{A}\left(\text { fault, } x_{p}, 0, c, \mu, \lambda, U, \delta, y_{p}, z\right)}
\end{aligned}
$$

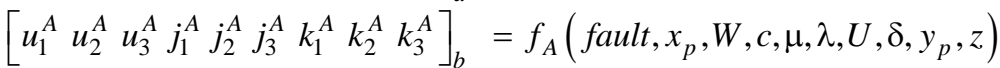

$$
\begin{aligned}
& {\left[u_{1}^{A} u_{2}^{A} u_{3}^{A} j_{1}^{A} j_{2}^{A} j_{3}^{A} k_{1}^{A} k_{2}^{A} k_{3}^{A}\right]_{c}=f_{A}\left(\text { fault, } x_{p}-L, 0, c, \mu, \lambda, U, \delta, y_{p}, z\right)}
\end{aligned}
$$

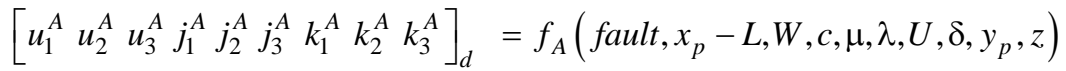

2. For an infinite medium at depth $-Z$ (equations for $f_{A}$ are in tables 18-21, p. 58-59),

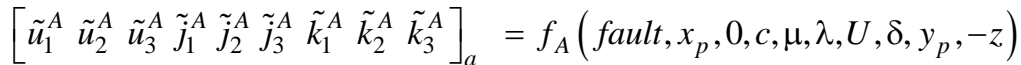

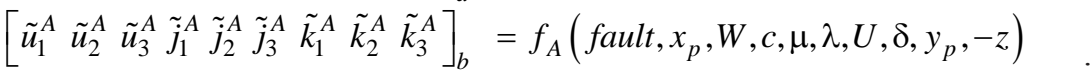

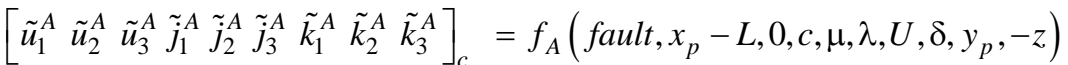

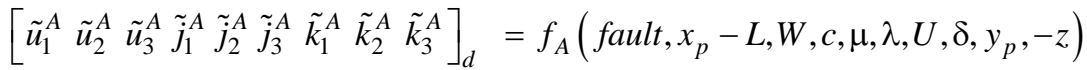

3. The free-surface deformation (equations for $f_{B}$ are in tables 22-25, p. 60-61)

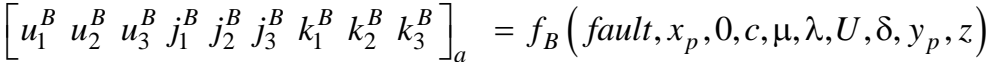

$$
\begin{aligned}
& {\left[\begin{array}{lllllllll}
u_{1}^{B} & u_{2}^{B} & u_{3}^{B} & j_{1}^{B} & j_{2}^{B} & j_{3}^{B} & k_{1}^{B} & k_{2}^{B} & k_{3}^{B}
\end{array}\right]_{b}=f_{B}\left(\text { fault }, x_{p}, W, c, \mu, \lambda, U, \delta, y_{p}, z\right)}
\end{aligned}
$$

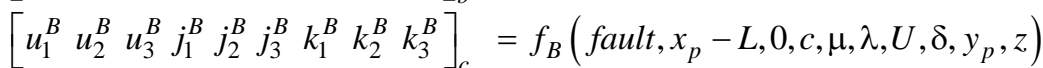

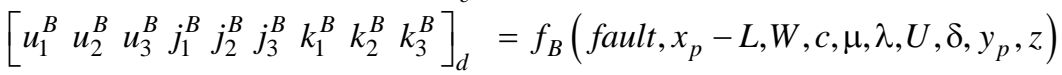

4. The depth-multiplied term (equations for $f_{C}$ are in tables 26-29, p. 62-64)

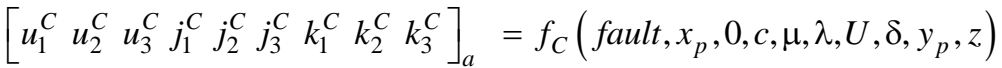

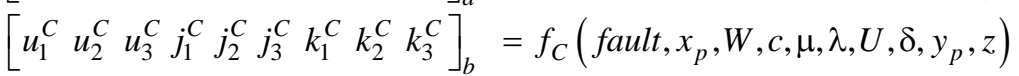

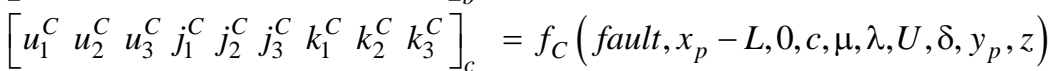

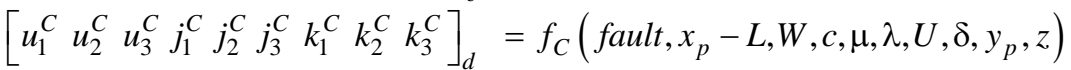


Step 2-Combination of Values

1. For, $u_{A}$, an infinite medium at depth $z$,

$$
\begin{aligned}
& u_{1}^{A}=\left[u_{1}^{A}\right]_{a}-\left[u_{1}^{A}\right]_{b}-\left[u_{1}^{A}\right]_{c}+\left[u_{1}^{A}\right]_{d} \\
& u_{2}^{A}=\left[u_{2}^{A}\right]_{a}-\left[u_{2}^{A}\right]_{b}-\left[u_{2}^{A}\right]_{c}+\left[u_{2}^{A}\right]_{d} . \\
& u_{3}^{A}=\left[u_{3}^{A}\right]_{a}-\left[u_{3}^{A}\right]_{b}-\left[u_{3}^{A}\right]_{c}+\left[u_{3}^{A}\right]_{d}
\end{aligned}
$$

2. For, $\tilde{u}_{A}$, an infinite medium at depth $-z$,

$$
\begin{aligned}
& \tilde{u}_{1}^{A}=\left[\tilde{u}_{1}^{A}\right]_{a}-\left[\tilde{u}_{1}^{A}\right]_{b}-\left[\tilde{u}_{1}^{A}\right]_{c}+\left[\tilde{u}_{1}^{A}\right]_{d} \\
& \tilde{u}_{2}^{A}=\left[\tilde{u}_{2}^{A}\right]_{a}-\left[\tilde{u}_{2}^{A}\right]_{b}-\left[\tilde{u}_{2}^{A}\right]_{c}+\left[\tilde{u}_{2}^{A}\right]_{d} . \\
& \tilde{u}_{3}^{A}=\left[\tilde{u}_{3}^{A}\right]_{a}-\left[\tilde{u}_{3}^{A}\right]_{b}-\left[\tilde{u}_{3}^{A}\right]_{c}+\left[\tilde{u}_{3}^{A}\right]_{d}
\end{aligned}
$$

3. For, the free-surface deformation,

$$
\begin{aligned}
& u_{1}^{B}=\left[u_{1}^{B}\right]_{a}-\left[u_{1}^{B}\right]_{b}-\left[u_{1}^{B}\right]_{c}+\left[u_{1}^{B}\right]_{d} \\
& u_{2}^{B}=\left[u_{2}^{B}\right]_{a}-\left[u_{2}^{B}\right]_{b}-\left[u_{2}^{B}\right]_{c}+\left[u_{2}^{B}\right]_{d} . \\
& u_{3}^{B}=\left[u_{3}^{B}\right]_{a}-\left[u_{3}^{B}\right]_{b}-\left[u_{3}^{B}\right]_{c}+\left[u_{3}^{B}\right]_{d}
\end{aligned}
$$

4. For, $u_{C}$, the depth-multiplied term,

$$
\begin{aligned}
& u_{1}^{C}=\left[u_{1}^{C}\right]_{a}-\left[u_{1}^{C}\right]_{b}-\left[u_{1}^{C}\right]_{c}+\left[u_{1}^{C}\right]_{d} \\
& u_{2}^{C}=\left[u_{2}^{C}\right]_{a}-\left[u_{2}^{C}\right]_{b}-\left[u_{2}^{C}\right]_{C}+\left[u_{2}^{C}\right]_{d} . \\
& u_{3}^{C}=\left[u_{3}^{C}\right]_{a}-\left[u_{3}^{C}\right]_{b}-\left[u_{3}^{C}\right]_{C}+\left[u_{3}^{C}\right]_{d}
\end{aligned}
$$

Step 3-Computation of Deformation in Okada's Coordinate System (1992)

$$
\begin{aligned}
& U_{x}^{p}=u_{1}^{A}-\tilde{u}_{1}^{A}+u_{1}^{B}+z \cdot u_{1}^{C} \\
& U_{y}^{p}=\left(u_{2}^{A}-\tilde{u}_{2}^{A}+u_{2}^{B}+z \cdot u_{2}^{C}\right) \cos \delta-\left(u_{3}^{A}-\tilde{u}_{3}^{A}+u_{3}^{B}+z \cdot u_{3}^{C}\right) \sin \delta . \\
& U_{z}^{p}=\left(u_{2}^{A}-\tilde{u}_{2}^{A}+u_{2}^{B}-z \cdot u_{2}^{C}\right) \sin \delta+\left(u_{3}^{A}-\tilde{u}_{3}^{A}+u_{3}^{B}-z \cdot u_{3}^{C}\right) \cos \delta
\end{aligned}
$$

Step 4-Rotate Deformation Back to Cartesian Coordinate System

$$
\begin{aligned}
& u_{x}=\sin \phi \cdot U_{x}^{p}-\cos \phi \cdot U_{y}^{p} \\
& u_{y}=\sin \phi \cdot U_{y}^{p}+\cos \phi \cdot U_{x}^{p} . \\
& u_{z}=U_{z}^{p}
\end{aligned}
$$




\section{Displacement-Gradient Matrix}

To compute the displacement-gradient matrix, follow a procedure similar to the one described in the previous section (equations 95-98).

\section{Combination of Values for X-Derivatives}

These equations are based Okada's equations (1992, table 7).

For, $j_{A}$, an infinite medium at depth $z$

$$
\begin{aligned}
& j_{1}^{A}=\left[j_{1}^{A}\right]_{a}-\left[j_{1}^{A}\right]_{b}-\left[j_{1}^{A}\right]_{c}+\left[j_{1}^{A}\right]_{d} \\
& j_{2}^{A}=\left[j_{2}^{A}\right]_{a}-\left[j_{2}^{A}\right]_{b}-\left[j_{2}^{A}\right]_{c}+\left[j_{2}^{A}\right]_{d} \\
& j_{3}^{A}=\left[j_{3}^{A}\right]_{a}-\left[j_{3}^{A}\right]_{b}-\left[j_{3}^{A}\right]_{c}+\left[j_{3}^{A}\right]_{d}
\end{aligned}
$$

For, $\tilde{j}_{A}$, an infinite medium at depth $-z$ (mirror image),

$$
\begin{aligned}
& \tilde{j}_{1}^{A}=\left[\tilde{j}_{1}^{A}\right]_{a}-\left[\tilde{j}_{1}^{A}\right]_{b}-\left[\tilde{j}_{1}^{A}\right]_{c}+\left[\tilde{j}_{1}^{A}\right]_{d} \\
& \tilde{j}_{2}^{A}=\left[\tilde{j}_{2}^{A}\right]_{a}-\left[\tilde{j}_{2}^{A}\right]_{b}-\left[\tilde{j}_{2}^{A}\right]_{c}+\left[\tilde{j}_{2}^{A}\right]_{d} . \\
& \tilde{j}_{3}^{A}=\left[\tilde{j}_{3}^{A}\right]_{a}-\left[\tilde{j}_{3}^{A}\right]_{b}-\left[\tilde{j}_{3}^{A}\right]_{c}+\left[\tilde{j}_{3}^{A}\right]_{d}
\end{aligned}
$$

For, $j_{B}$, the free-surface deformation,

$$
\begin{aligned}
& j_{1}^{B}=\left[j_{1}^{B}\right]_{a}-\left[j_{1}^{B}\right]_{b}-\left[j_{1}^{B}\right]_{c}+\left[j_{1}^{B}\right]_{d} \\
& j_{2}^{B}=\left[j_{2}^{B}\right]_{a}-\left[j_{2}^{B}\right]_{b}-\left[j_{2}^{B}\right]_{c}+\left[j_{2}^{B}\right]_{d} \\
& j_{3}^{B}=\left[j_{3}^{B}\right]_{a}-\left[j_{3}^{B}\right]_{b}-\left[j_{3}^{B}\right]_{C}+\left[j_{3}^{B}\right]_{d}
\end{aligned}
$$

For, $j_{C}$, the depth-multiplied term,

$$
\begin{aligned}
& j_{1}^{C}=\left[j_{1}^{C}\right]_{a}-\left[j_{1}^{C}\right]_{b}-\left[j_{1}^{C}\right]_{C}+\left[j_{1}^{C}\right]_{d} \\
& j_{2}^{C}=\left[j_{2}^{C}\right]_{a}-\left[j_{2}^{C}\right]_{b}-\left[j_{2}^{C}\right]_{C}+\left[j_{2}^{C}\right]_{d} . \\
& j_{3}^{C}=\left[j_{3}^{C}\right]_{a}-\left[j_{3}^{C}\right]_{b}-\left[j_{3}^{C}\right]_{C}+\left[j_{3}^{C}\right]_{d}
\end{aligned}
$$

\section{Combination of Values for Y-Derivatives (Okada 1992, table 8)}

For, $k_{A}$, an infinite medium at depth $z$,

$$
\begin{aligned}
& k_{1}^{A}=\left[k_{1}^{A}\right]_{a}-\left[k_{1}^{A}\right]_{b}-\left[k_{1}^{A}\right]_{c}+\left[k_{1}^{A}\right]_{d} \\
& k_{2}^{A}=\left[k_{2}^{A}\right]_{a}-\left[k_{2}^{A}\right]_{b}-\left[k_{2}^{A}\right]_{c}+\left[k_{2}^{A}\right]_{d} . \\
& k_{3}^{A}=\left[k_{3}^{A}\right]_{a}-\left[k_{3}^{A}\right]_{b}-\left[k_{3}^{A}\right]_{c}+\left[k_{3}^{A}\right]_{d}
\end{aligned}
$$


For, $\tilde{k}_{A}$, an infinite medium at depth $-z$ (mirror image),

$$
\begin{aligned}
& \tilde{k}_{1}^{A}=\left[\tilde{k}_{1}^{A}\right]_{a}-\left[\tilde{k}_{1}^{A}\right]_{b}-\left[\tilde{k}_{1}^{A}\right]_{c}+\left[\tilde{k}_{1}^{A}\right]_{d} \\
& \tilde{k}_{2}^{A}=\left[\tilde{k}_{2}^{A}\right]_{a}-\left[\tilde{k}_{2}^{A}\right]_{b}-\left[\tilde{k}_{2}^{A}\right]_{c}+\left[\tilde{k}_{2}^{A}\right]_{d} . \\
& \tilde{k}_{3}^{A}=\left[\tilde{k}_{3}^{A}\right]_{a}-\left[\tilde{k}_{3}^{A}\right]_{b}-\left[\tilde{k}_{3}^{A}\right]_{c}+\left[\tilde{k}_{3}^{A}\right]_{d}
\end{aligned}
$$

For, $k_{B}$, the free-surface deformation,

$$
\begin{aligned}
& k_{1}^{B}=\left[k_{1}^{B}\right]_{a}-\left[k_{1}^{B}\right]_{b}-\left[k_{1}^{B}\right]_{c}+\left[k_{1}^{B}\right]_{d} \\
& k_{2}^{B}=\left[k_{2}^{B}\right]_{a}-\left[k_{2}^{B}\right]_{b}-\left[k_{2}^{B}\right]_{c}+\left[k_{2}^{B}\right]_{d} . \\
& k_{3}^{B}=\left[k_{3}^{B}\right]_{a}-\left[k_{3}^{B}\right]_{b}-\left[k_{3}^{B}\right]_{c}+\left[k_{3}^{B}\right]_{d}
\end{aligned}
$$

For, $k_{C}$, the depth-multiplied term,

$$
\begin{aligned}
& k_{1}^{C}=\left[k_{1}^{C}\right]_{a}-\left[k_{1}^{C}\right]_{b}-\left[k_{1}^{C}\right]_{C}+\left[k_{1}^{C}\right]_{d} \\
& k_{2}^{C}=\left[k_{2}^{C}\right]_{a}-\left[k_{2}^{C}\right]_{b}-\left[k_{2}^{C}\right]_{C}+\left[k_{2}^{C}\right]_{d} . \\
& k_{3}^{C}=\left[k_{3}^{C}\right]_{a}-\left[k_{3}^{C}\right]_{b}-\left[k_{3}^{C}\right]_{C}+\left[k_{3}^{C}\right]_{d}
\end{aligned}
$$

Computation of $\mathrm{X}$ and $\mathrm{Y}$ Derivatives

$$
\begin{aligned}
& \frac{\partial U p_{x}}{\partial x}=j_{1}^{A}-\tilde{j}_{1}^{A}+j_{1}^{B}+z \cdot j_{1}^{C} \\
& \frac{\partial U p_{y}}{\partial x}=\left(j_{2}^{A}-\tilde{j}_{2}^{A}+j_{2}^{B}+z \cdot j_{2}^{C}\right) \cos \delta-\left(j_{3}^{A}-\tilde{j}_{3}^{A}+j_{3}^{B}+z \cdot j_{3}^{C}\right) \sin \delta \cdot \\
& \frac{\partial U p_{z}}{\partial x}=\left(j_{2}^{A}-\tilde{j}_{2}^{A}+j_{2}^{B}-z \cdot j_{2}^{C}\right) \sin \delta+\left(j_{3}^{A}-\tilde{j}_{3}^{A}+j_{3}^{B}-z \cdot j_{3}^{C}\right) \cos \delta \\
& \frac{\partial U p_{x}}{\partial y}=k_{1}^{A}-\tilde{k}_{1}^{A}+k_{1}^{B}+z \cdot k_{1}^{C} \\
& \frac{\partial U p_{y}}{\partial y}=\left(k_{2}^{A}-\tilde{k}_{2}^{A}+k_{2}^{B}+z \cdot k_{2}^{C}\right) \cos \delta-\left(k_{3}^{A}-\tilde{k}_{3}^{A}+k_{3}^{B}+z \cdot k_{3}^{C}\right) \sin \delta \cdot \\
& \frac{\partial U p_{z}}{\partial y}=\left(k_{2}^{A}-\tilde{k}_{2}^{A}+k_{2}^{B}-z \cdot k_{2}^{C}\right) \sin \delta+\left(k_{3}^{A}-\tilde{k}_{3}^{A}+k_{3}^{B}-z \cdot k_{3}^{C}\right) \cos \delta
\end{aligned}
$$

Rotation of the Displacement-Gradient Matrix Back to Cartesian Coordinate System

$$
\begin{aligned}
& \frac{\partial u}{\partial x}=\frac{\partial U p_{x}}{\partial x} \sin ^{2} \phi-\left(\frac{\partial U p_{x}}{\partial y}+\frac{\partial U p_{y}}{\partial x}\right) \sin \phi \cos \phi+\frac{\partial U p_{y}}{\partial y} \cos ^{2} \phi \\
& \frac{\partial u}{\partial y}=\frac{\partial U p_{x}}{\partial y} \sin ^{2} \phi+\left(\frac{\partial U p_{x}}{\partial x}-\frac{\partial U p_{y}}{\partial y}\right) \sin \phi \cos \phi-\frac{\partial U p_{y}}{\partial x} \cos ^{2} \phi \\
& \frac{\partial v}{\partial x}=\frac{\partial U p_{y}}{\partial x} \sin ^{2} \phi+\left(\frac{\partial U p_{x}}{\partial x}-\frac{\partial U p_{y}}{\partial y}\right) \sin \phi \cos \phi-\frac{\partial U p_{x}}{\partial y} \cos ^{2} \phi \\
& \frac{\partial v}{\partial y}=\frac{\partial U p_{y}}{\partial y} \sin ^{2} \phi+\left(\frac{\partial U p_{x}}{\partial y}+\frac{\partial U p_{y}}{\partial x}\right) \sin \phi \cos \phi+\frac{\partial U p_{x}}{\partial x} \cos ^{2} \phi
\end{aligned}
$$




\section{Strain}

The three strain components that describe the horizontal strain tensor are the areal strain $\varepsilon_{a}$, and $\gamma_{1}$ and $\gamma_{2}$ shear strains:

$$
\begin{aligned}
\varepsilon_{a} & =0.001\left(\varepsilon_{x x}+\varepsilon_{y y}\right) \\
\gamma_{1} & =0.001\left(\varepsilon_{x x}-\varepsilon_{y y}\right), \\
\gamma_{2} & =0.001 \cdot 2 \varepsilon_{x y}
\end{aligned}
$$

where

$$
\varepsilon_{x x}=\frac{\partial u}{\partial x}, \quad \varepsilon_{y y}=\frac{\partial v}{\partial y} \text { and } \quad \varepsilon_{x y}=\varepsilon_{y x}=\frac{1}{2}\left(\frac{\partial u}{\partial y}+\frac{\partial v}{\partial x}\right)
$$

The components of the displacement-gradient tensor are given in equation 111 . The scale 0.001 in equation 112 takes into account that, in Okada (1992), coordinates are in kilometers and displacements in meters.

\section{General Parameters}

The parameters and variables listed below are the same for $f_{A}, f_{B}$ and $f_{C}$. They are listed in the order in which they would be coded:

Okada (1992, table 6)

$$
\begin{gathered}
\alpha=\frac{\lambda+\mu}{\lambda+2 \mu}=\frac{1}{2} \frac{1}{1-v}, \quad \alpha_{1}=\frac{1-\alpha}{\alpha} \\
p=y_{p} \cos \delta+d \sin \delta \quad q=y_{p} \sin \delta-d \cos \delta \\
\eta=p-W \\
R^{2}=\xi^{2}+\eta^{2}+q^{2} \\
\tilde{y}=\eta \cos \delta+q \sin \delta, \quad \tilde{d}=\eta \sin \delta-q \cos \delta \\
d=c-z, \quad \tilde{c}=\tilde{d}+z
\end{gathered}
$$

where $\xi$ and $\eta$ are dummy integration variables used the first time in equations 68 ; their lower and limits are defined in equations 91-94. For example, $f_{A}=f_{A}\left(\right.$ fault $\left., \xi, \eta, c, \mu, \lambda, U, \delta, y_{p}, z\right)$.

Okada (1992, equation 14; subscripts from tables 6-8)

$$
\begin{gathered}
X_{11}=\frac{1}{R(R+\xi)}, \quad X_{32}=\frac{2 R+\xi}{R^{3}(R+\xi)^{2}}, \quad X_{53}=\frac{8 R^{2}+9 R \xi+3 \xi^{2}}{R^{5}(R+\xi)^{3}} \\
Y_{11}=\frac{1}{R(R+\eta)}, \quad Y_{32}=\frac{2 R+\eta}{R^{3}(R+\eta)^{2}}, \quad Y_{53}=\frac{8 R^{2}+9 R \eta+3 \eta^{2}}{R^{5}(R+\eta)^{3}} \\
h=q \cos \delta-z \\
Z_{32}=\frac{\sin \delta}{R^{3}}-h Y_{32}, \quad Z_{53}=\frac{3 \sin \delta}{R^{5}}-h Y_{53} \\
Y_{0}=Y_{11}-\xi^{2} Y_{32}, \quad Z_{0}=Z_{32}-\xi^{2} Z_{53}
\end{gathered}
$$


Okada (1992, footnote, table 6)

$$
\begin{aligned}
& F_{0}=0, \\
& c_{1}=|q| \leq 0, \\
& c_{2}=|q|>0 \text { and } \\
& \Theta=c_{1} F_{0}+c_{2} \tan ^{-1}\left(\frac{\xi \eta}{q R}\right),
\end{aligned}
$$

where

$$
\begin{array}{ll}
q & \text { is a vector; } \\
F_{0} & \text { is a vector of zeros the same size of } q ; \\
c_{1} \text { and } c_{2} & \text { are logical vectors (a logical vector is equal to } 1 \text { if a logical } \\
& \text { condition is satisfied and is equal to } 0 \text { otherwise). }
\end{array}
$$

Singularity Condition iii (Okada,1992, p. 1034)

$$
\begin{gathered}
F_{0}=0 \\
c_{1}=|R+\xi| \leq 0 \\
c_{2}=|R+\xi|>0 \\
R_{\xi}=c_{1} F_{0}+c_{2} \frac{1}{R+\xi} \\
\ln R_{\xi}=-c_{1} \ln (R-\xi)+c_{2} \ln (R+\xi) ;
\end{gathered}
$$

where $F_{0}$ is a dummy vector of zeros the same size as $R+\xi$, and $c_{1}$ and $c_{2}$ are dummy $\operatorname{logical}$ vectors.

Singularity Condition iv (Okada,1992, p. 1034)

$$
\begin{aligned}
F_{0} & =0 \\
c_{1} & =|R+\eta| \leq 0 \\
c_{2} & =|R+\eta|>0 \\
\mathrm{R}_{\eta} & =c_{1} F_{0}+c_{2} \frac{1}{R+\eta} \\
\ln R_{\eta} & =-c_{1} \ln (R-\eta)+c_{2} \ln (R+\eta) ;
\end{aligned}
$$

where $F_{0}$ is a dummy vector of zeros the same size as $R+\eta$, and $c_{1}$ and $c_{2}$ are dummy $\operatorname{logical}$ vectors. 
I Parameters (Okada, 1992, footnote, table 6)

Singularity Condition ii (Okada,1992, p. 1034)

$$
\begin{aligned}
F_{0} & =0 \\
c_{1} & =|\xi| \leq 0 \\
c_{2} & =|\xi|>0 \\
X & =\sqrt{\xi^{2}+q^{2}}
\end{aligned}
$$

where $F_{0}$ is a dummy vector of zeros the same size of $R+\eta$, and $c_{1}$ and $c_{2}$ are dummy logical vectors.

$$
\begin{aligned}
& I_{3}=\frac{1}{\cos \delta} \frac{\tilde{y}}{R+\tilde{d}}-\frac{1}{\cos ^{2} \delta}[\ln (R+\eta)-\sin \delta \ln (R+\tilde{d})] \\
& I_{4}=c_{1} F_{0}+c_{2}\left[\frac{\sin \delta}{\cos \delta} \frac{\xi}{R+\tilde{d}}+\frac{2}{\cos ^{2} \delta} \tan ^{-1}\left(\frac{\eta(X+q \cos \delta)+X(R+X) \sin \delta}{\xi(R+X) \cos \delta}\right)\right] \\
& I_{1}=-\frac{\xi}{R+\tilde{d}} \cos \delta-I_{4} \sin \delta \\
& I_{2}=\log (R+\tilde{d})+I_{3} \sin \delta
\end{aligned}
$$

if $\cos \delta=0$, then

$$
\begin{aligned}
& I_{3}=0.5\left[\frac{\eta}{R+\tilde{d}}+\frac{\tilde{y} q}{(R+\tilde{d})^{2}}-\ln (R+\eta)\right] \\
& I_{4}=c_{1} F_{0}+c_{2} \cdot 0.5 \frac{\xi \tilde{y}}{(R+\tilde{d})^{2}}
\end{aligned}
$$

J and K Parameters (Okada, 1992, footnote, table 7)

$$
\begin{aligned}
D_{11} & =\frac{1}{R(R+\tilde{d})} \\
J_{2} & =\frac{\xi \tilde{y}}{R+\tilde{d}} D_{11} \\
J_{5} & =-\left(\tilde{d}+\frac{\tilde{y}^{2}}{R+\tilde{d}}\right) D_{11}
\end{aligned}
$$




$$
\begin{gathered}
K_{1}=\xi \frac{D_{11}-Y_{11} \sin \delta}{\cos \delta} \\
K_{3}=\frac{q Y_{11}-\tilde{y} D_{11}}{\cos \delta}, \\
J_{3}=\frac{K_{1}-J_{2} \sin \delta}{\cos \delta} \\
J_{6}=\frac{K_{3}-J_{5} \sin \delta}{\cos \delta} \\
J_{1}=J_{5} \cos \delta-J_{6} \sin \delta \\
J_{4}=-\xi Y_{11}-J_{2} \cos \delta+J_{3} \sin \delta \\
K_{2}=\frac{1}{R}+K_{3} \sin \delta \\
K_{4}=\xi Y_{11} \cos \delta-K_{1} \sin \delta
\end{gathered} ;
$$

if $\cos \delta=0$,

$$
\begin{aligned}
& K_{1}=\frac{\xi q D_{11}}{R+\tilde{d}} \\
& K_{3}=\sin \delta \frac{\xi^{2} \cdot * D_{11}-1}{R+\tilde{d}} \\
& J_{3}=-\xi \frac{q^{2} D_{11}-0.5}{(R+\tilde{d})^{2}} . \\
& J_{6}=-\tilde{y} \frac{\xi^{2} D_{11}-0.5}{(R+\tilde{d})^{2}}
\end{aligned}
$$

Parameters E-0 (Okada, 1992, footnote, table 8)

$$
\begin{aligned}
E & =\frac{\sin \delta}{R}-\frac{\tilde{y} q}{R^{3}} \\
F & =\frac{\tilde{d}}{R^{3}}+\xi^{2} Y_{32} \sin \delta \\
G & =2 X_{11} \sin \delta-\tilde{y} q X_{32} \\
H & =\tilde{d} q X_{32}+\xi q Y_{32} \sin \delta \\
P & =\frac{\cos \delta}{R^{3}}+q Y_{32} \sin \delta \\
Q & =3 \frac{\tilde{c} \tilde{d}}{R^{5}}-\left(z Y_{32}+Z_{32}+Z_{0}\right) \sin \delta
\end{aligned}
$$




\section{Function $f_{A}$ (Infinite Medium)}

Table 18. Strike-slip fault (internal deformation and strain; function $f_{A}$ )

[Okada (1992), type strike - for displacement, see table 6; for X-derivative, see table 7, and for Y-derivative, see table 8]

\begin{tabular}{ll}
\hline \multicolumn{1}{c}{ Displacement } \\
\hline$u_{1}^{A}$ & $0.5\left(\Theta+\alpha \xi q Y_{11}\right)$ \\
$u_{2}^{A}$ & $0.5 \alpha \mathrm{q} / R$ \\
$u_{3}^{A}$ & $0.5(1-\alpha) \ln (R+\eta)-0.5 \alpha q^{2} Y_{11}$ \\
\hline & X-derivative \\
\hline$j_{1}^{A}$ & $-0.5(1-\alpha) q Y_{11}-0.5 \alpha \xi^{2} q Y_{32}$ \\
$j_{2}^{A}$ & $-0.5 \alpha \xi q / R^{3}$ \\
$j_{3}^{A}$ & $0.5(1-\alpha) \xi Y_{11}+0.5 \alpha \xi q^{2} Y_{32}$ \\
\hline & Y-derivative \\
\hline$k_{1}^{A}$ & $0.5(1-\alpha) \xi Y_{11} \sin \delta+0.5 \tilde{d} X_{11}+0.5 \alpha \xi F$ \\
$k_{2}^{A}$ & $0.5 \alpha \mathrm{E}$ \\
$k_{3}^{A}$ & $0.5(1-\alpha)\left(\cos \delta / R+q Y_{11} \sin \delta\right)-0.5 \alpha q F$ \\
\hline
\end{tabular}

Table 19. Dip-slip fault (internal deformation and strain; function $f_{A}$ )

[Okada (1992), type dip — for displacement, see table 6; for X-derivative, see table 7, and for Y-derivative, see table 8]

\begin{tabular}{ll}
\hline \multicolumn{1}{c}{ Displacement } \\
\hline$u_{1}^{A}$ & $0.5 \alpha q / R$ \\
$u_{2}^{A}$ & $0.5 \Theta+0.5 \alpha \eta q X_{11}$ \\
$u_{3}^{A}$ & $0.5(1-\alpha) \ln (R+\xi)-0.5 \alpha q^{2} X_{11}$ \\
\hline & X-derivative \\
\hline$j_{1}^{A}$ & $-0.5 \alpha \xi q / R^{3}$ \\
$j_{2}^{A}$ & $-0.5 q Y_{11}-0.5 \alpha \eta q / R^{3}$ \\
$j_{3}^{A}$ & $0.5(1-\alpha) / R+0.5 \alpha q^{2} / R^{3}$ \\
\hline & Y-derivative \\
$k_{1}^{A}$ & $0.5 \alpha E$ \\
$k_{2}^{A}$ & $0.5(1-\alpha) \tilde{d} X_{11}+0.5 \xi Y_{11} \sin \delta+0.5 \alpha \eta G$ \\
$k_{3}^{A}$ & $0.5(1-\alpha) \tilde{y} X_{11}-0.5 \alpha q G$
\end{tabular}


Table 20. Tensile crack (internal deformation and strain; function $f_{A}$ )

[Okada (1992), type tensile—-for displacement, see table 6; for X-derivative, see table 7, and for Y-derivative, see table 8]

\begin{tabular}{cc}
\hline & Displacement \\
\hline$u_{1}^{A}$ & $-0.5(1-\alpha) \ln (R+\eta)-0.5 \alpha q^{2} Y_{11}$ \\
$u_{2}^{A}$ & $-0.5(1-\alpha) \ln (R+\xi)-0.5 \alpha q^{2} X_{11}$ \\
$u_{3}^{A}$ & $0.5 \Theta-0.5 \alpha q\left(\eta X_{11}+\xi Y_{11}\right)$ \\
\hline & X-derivative \\
\hline$j_{1}^{A}$ & $-0.5(1-\alpha) \xi Y_{11}+0.5 \alpha \xi q^{2} Y_{32}$ \\
$j_{2}^{A}$ & $-0.5(1-\alpha) / R+0.5 \alpha q^{2} / R^{3}$ \\
$j_{3}^{A}$ & $-0.5(1-\alpha) q Y_{11}-0.5 \alpha q^{3} Y_{32}$ \\
\hline & $-0.5(1-\alpha)\left(\cos \delta / R+q Y_{11} \sin \delta\right)-0.5 \alpha q F[$ \\
$k_{1}^{A}$ & $-0.5(1-\alpha) \tilde{y} X_{11}-0.5 \alpha q G$ \\
$k_{2}^{A}$ & $0.5(1-\alpha)\left(\tilde{d} X_{11}+\xi Y_{11} \sin \delta\right)+0.5 \alpha q H$ \\
$k_{3}^{A}$ &
\end{tabular}

Table 21. Scaling used to compute the dimensional displacement (function $f_{A}$ ).

[Okada (1992), i=1: strike-slip; i=2: dip-slip; i=3: tensile e-for displacement, see table 6; for X-derivative, see table 7, and for Y-derivative, see table 8]

\begin{tabular}{cc}
\hline \multicolumn{2}{c}{ Displacement (Okada, 1992; Table 6) } \\
\hline$u_{1}^{A}$ & $\left(0.5 U_{i} / \pi\right) u_{1}^{A}$ \\
$u_{2}^{A}$ & $\left(0.5 U_{i} / \pi\right) u_{2}^{A}$ \\
$u_{3}^{A}$ & $\left(0.5 U_{i} / \pi\right) u_{3}^{A}$ \\
\hline \multicolumn{2}{c}{ X-Derivative (0kada, 1992; Table 7) } \\
\hline$j_{1}^{A}$ & $\left(0.5 U_{i} / \pi\right) j_{1}^{A}$ \\
$j_{2}^{A}$ & $\left(0.5 U_{i} / \pi\right) j_{2}^{A}$ \\
$j_{3}^{A}$ & $\left(0.5 U_{i} / \pi\right) j_{3}^{A}$ \\
\hline \multicolumn{2}{c}{ Y-Derivative (0kada, 1992; Table 8) } \\
\hline$k_{1}^{A}$ & $\left(0.5 U_{i} / \pi\right) k_{1}^{A}$ \\
$k_{2}^{A}$ & $\left(0.5 U_{i} / \pi\right) k_{2}^{A}$ \\
$k_{3}^{A}$ & $\left(0.5 U_{i} / \pi\right) k_{3}^{A}$ \\
\hline
\end{tabular}




\section{Function $f_{B}$ (Free Surface Deformation)}

Table 22. Strike-slip fault (internal deformation and strain; function $f_{B}$ ).

[Okada (1992), type strike - for displacement, see table 6; for X-derivative, see table 7, and for Y-derivative, see table 8]

\begin{tabular}{cl}
\hline & Displacement \\
\hline$u_{1}^{B}$ & $-\xi q Y_{11}-\Theta-\alpha_{1} I_{1} \sin \delta$ \\
$u_{2}^{B}$ & $-q / R+\alpha_{1} \tilde{y} \sin \delta /(R+\tilde{d})$ \\
$u_{3}^{B}$ & $q^{2} Y_{11}-\alpha_{1} I_{2} \sin \delta$ \\
\hline & $\mathbf{X}$-derivative \\
\hline$j_{1}^{B}$ & $\xi^{2} q Y_{32}-\alpha_{1} J_{1} \sin \delta$ \\
$j_{2}^{B}$ & $\xi q / R^{3}-\alpha_{1} J_{2} \sin \delta$ \\
$j_{3}^{B}$ & $-\xi q^{2} Y_{32}-\alpha_{1} J_{3} \sin \delta$ \\
\hline & $\mathbf{Y}$-derivative \\
$k_{1}^{B}$ & $-\xi F-\tilde{d} X_{11}+\alpha_{1}\left(\xi Y_{11}+J_{4}\right) \sin \delta$ \\
$k_{2}^{B}$ & $-E+\alpha_{1}\left(1 / R+J_{5}\right) \sin \delta$ \\
$k_{3}^{B}$ & $q F-\alpha_{1}\left(q Y_{11}-J_{6}\right) \sin \delta$ \\
\hline
\end{tabular}

Table 23. Dip-slip fault (internal deformation and strain; function $f_{B}$ ).

[Okada (1992), type dip—for displacement, see table 6; for X-derivative, see table 7, and for Y-derivative, see table 8]

\begin{tabular}{|c|c|}
\hline \multicolumn{2}{|r|}{ Displacement } \\
\hline$u_{1}^{B}$ & $-q / R+((1-\alpha) / \alpha) I_{3} \sin \delta \cos \delta$ \\
\hline$u_{2}^{B}$ & $-\eta q X_{11}-\Theta-((1-\alpha) / \alpha)(\xi /(R+\tilde{d})) \sin \delta \cos \delta$ \\
\hline$u_{3}^{B}$ & $q^{2} X_{11}+((1-\alpha) / \alpha) I_{4} \sin \delta \cos \delta$ \\
\hline \multicolumn{2}{|r|}{ X-derivative } \\
\hline$j_{1}^{B}$ & $\xi q / R^{3}+\alpha_{1} J_{4} \sin \delta \cos \delta$ \\
\hline$j_{2}^{B}$ & $\eta q / R^{3}+q Y_{11}+\alpha_{1} J_{5} \sin \delta \cos \delta$ \\
\hline$j_{3}^{B}$ & $-q^{2} / R^{3}+\alpha_{1} J_{6} \sin \delta \cos \delta$ \\
\hline \multicolumn{2}{|r|}{ Y-derivative } \\
\hline$k_{1}^{B}$ & $-E+\alpha_{1} J_{1} \sin \delta \cos \delta$ \\
\hline$k_{2}^{B}$ & $-\eta G-\xi Y_{11} \sin \alpha+\alpha_{1} J_{2} \sin \delta \cos \delta$ \\
\hline$k_{3}^{B}$ & $q G+\alpha_{1} J_{3} \sin \delta \cos \delta$ \\
\hline
\end{tabular}


Table 24. Tensile crack (internal deformation and strain; function $f_{B}$ ).

[Okada (1992), type tensile - for displacement, see table 6; for X-derivative, see table 7, and for Y-derivative, see table 8]

\begin{tabular}{cl}
\hline & Displacement \\
\hline$u_{1}^{B}$ & $q^{2} Y_{11}-\alpha_{1} I_{3} \sin ^{2} \delta$ \\
$u_{2}^{B}$ & $q^{2} X_{11}+\alpha_{1}(\xi /(R+$ dtilde $)) \sin ^{2} \delta$ \\
$u_{3}^{B}$ & $q\left(\eta X_{11}+\xi Y_{11}\right)-\Theta-\alpha_{1} I_{4} \sin ^{2} \delta$ \\
\hline & X-derivative \\
\hline$j_{1}^{B}$ & $-\xi q^{2} Y_{32}-\alpha_{1} J_{4} \sin ^{2} \delta$ \\
$j_{2}^{B}$ & $-q^{2} / R^{3}-\alpha_{1} J_{5} \sin ^{2} \delta$ \\
$j_{3}^{B}$ & $q^{3} Y_{32}-\alpha_{1} J_{6} \sin ^{2} \delta$ \\
\hline & $\mathbf{Y}$-derivative \\
$k_{1}^{B}$ & $q F-\alpha_{1} J_{1} \sin ^{2} \delta$ \\
$k_{2}^{B}$ & $q G-\alpha_{1} J_{2} \sin ^{2} \delta$ \\
$k_{3}^{B}$ & $-q H-\alpha_{1} J_{3} \sin ^{2} \delta$ \\
\hline
\end{tabular}

Table 25. Scaling (internal deformation and strain; function $f_{B}$ )

[Okada (1992), i=1: strike-slip; i=2: dip-slip; $\mathrm{i}=3$ : tensile e-for displacement, see table 6; for X-derivative, see table 7, and for Y-derivative, see table 8]

\begin{tabular}{cc}
\hline \multicolumn{2}{c}{ Displacement } \\
\hline$u_{1}^{B}$ & $\left(0.5 U_{i} / \pi\right) u_{1}^{B}$ \\
$u_{2}^{B}$ & $\left(0.5 U_{i} / \pi\right) u_{2}^{B}$ \\
$u_{3}^{B}$ & $\left(0.5 U_{i} / \pi\right) u_{3}^{B}$ \\
\hline & X-derivative \\
\hline$j_{1}^{B}$ & $\left(0.5 U_{i} / \pi\right) j_{1}^{B}$ \\
$j_{2}^{B}$ & $\left(0.5 U_{i} / \pi\right) j_{2}^{B}$ \\
$j_{3}^{B}$ & $\left(0.5 U_{i} / \pi\right) j_{3}^{B}$ \\
\hline$k_{1}^{B}$ & Y-derivative \\
$k_{2}^{B}$ & $\left(0.5 U_{i} / \pi\right) k_{1}^{B}$ \\
$k_{3}^{B}$ & $\left(0.5 U_{i} / \pi\right) k_{2}^{B}$ \\
\hline
\end{tabular}




\section{Function $f_{C}$ (Depth Multiplied Term)}

Table 26. Strike-slip fault (internal deformation and strain; function $f_{C}$ ).

[Okada (1992), type strike — for displacement, see table 6; for X-derivative, see table 7, and for Y-derivative, see table 8]

\begin{tabular}{cl}
\hline & Displacement \\
\hline$u_{1}^{C}$ & $(1-\alpha) \xi Y_{11} \cos \delta-\alpha \xi q Z_{32}$ \\
$u_{2}^{C}$ & $(1-\alpha)\left(\cos \delta / R+2 q Y_{11} \sin \delta\right)-\alpha \tilde{c} q / R^{3}$ \\
$u_{3}^{C}$ & $(1-\alpha) q Y_{11} \cos \delta-\alpha\left(\tilde{c} \eta / R^{3}-z Y_{11}+\xi^{2} Z_{32}\right)$ \\
\hline & $\quad$ X-derivative \\
\hline$j_{1}^{C}$ & $(1-\alpha) Y_{0} \cos \delta-\alpha q Z_{0}$ \\
$j_{2}^{C}$ & $-(1-\alpha) \xi\left(\cos \delta / R^{3}+2 q Y_{32} \sin \delta\right)+\alpha 3 \tilde{c} \xi q / R^{5}$ \\
$j_{3}^{C}$ & $-(1-\alpha) \xi q Y_{32} \cos \delta+\alpha \xi\left(3 \tilde{c} \eta / R^{5}-z Y_{32}-Z_{32}-Z_{0}\right)$ \\
\hline & $\quad$ Y-derivative \\
$k_{1}^{C}$ & $-(1-\alpha) \xi P \cos \delta-\alpha \xi Q$ \\
$k_{2}^{C}$ & $2(1-\alpha)\left(\tilde{d} R^{3}-Y_{0} \sin \delta\right) \sin \delta-\tilde{y} \cos \delta / R^{3}-\alpha\left[(\tilde{c}+\tilde{d}) \sin \delta / R^{3}-\eta / R^{3}-3 \tilde{c} \tilde{y} q / R^{5}\right]$ \\
$k_{3}^{C}$ & $-(1-\alpha) q / R^{3}+\left(\tilde{y} / R^{3}-Y_{0} \cos \delta\right) \sin \delta+\alpha\left[(\tilde{c}+\tilde{d}) \cos \delta / R^{3} /+3 \tilde{c} \tilde{d} q / R^{5}-\left(Y_{0} \cos \delta+q Z_{0}\right) \sin \delta\right]$ \\
\hline
\end{tabular}


Table 27. Dip-slip fault (internal deformation and strain; function $f_{C}$ ).

[Okada (1992), type dip —-for displacement, see table 6; for X-derivative, see table 7, and for Y-derivative, see table 8]

\begin{tabular}{cc}
\hline$u_{1}^{C}$ & Displacement \\
\hline$u_{2}^{C}$ & $(1-\alpha) \cos \delta / R-q Y_{11} \sin \delta-\alpha \tilde{c} q / R^{3}$ \\
$u_{3}^{C}$ & $(1-\alpha) \tilde{y} X_{11}-\alpha \tilde{c} \eta q X_{32}$ \\
\hline & $-\tilde{d} X_{11}-\xi Y_{11} \sin \delta-\alpha \tilde{c}\left(X_{11}-q^{2} X_{32}\right)$ \\
\hline$j_{1}^{C}$ & X-derivative \\
$j_{2}^{C}$ & $-(1-\alpha) \xi \cos \delta / R^{3}+\xi q Y_{32} \sin \delta+\alpha 3 \tilde{c} \xi q / R^{5}$ \\
$j_{3}^{C}$ & $-(1-\alpha) \tilde{y} / R^{3}+\alpha 3 \tilde{c} \eta q / R^{5}$ \\
\hline & $\tilde{d} / R^{3}-Y_{0} \sin \delta+\alpha \tilde{c}\left(1-3 q^{2} / R^{2}\right) / R^{3}$ \\
\hline$k_{1}^{C}$ & $-(1-\alpha) \eta / R^{3}+Y_{0} \sin ^{2} \delta-\alpha\left[(\tilde{c}+\tilde{d}) \sin \delta / R^{3}-3 \tilde{c} \tilde{y} q / R^{5}\right]$ \\
$k_{2}^{C}$ & $(1-\alpha)\left(X_{11}-\tilde{y}^{2} X_{32}\right)-\alpha \tilde{c}\left[(\tilde{d}+2 q \cos \delta) X_{32}-\tilde{y} \eta q X_{53}\right]$ \\
$k_{3}^{C}$ & $\xi P \sin \delta+\tilde{y} \tilde{d} X_{32}+\alpha \tilde{c}\left[(\tilde{y}+2 q \sin \delta) X_{32}-\tilde{y} q^{2} X_{53}\right]$ \\
\hline
\end{tabular}


Table 28. Tensile crack (internal deformation and strain; function $f_{C}$ ).

[Okada (1992), type tensile — for displacement, see table 6; for X-derivative, see table 7, and for Y-derivative, see table 8]

\begin{tabular}{lc}
\hline & Displacement \\
\hline$u_{1}^{C}$ & $-(1-\alpha)\left(\sin \delta / R+q Y_{11} \cos \delta\right)-\alpha\left(z Y_{11}-q^{2} Z_{32}\right)$ \\
$u_{2}^{C}$ & $(1-\alpha) 2 \xi Y_{11} \sin \delta+\tilde{d} X_{11}-\alpha \tilde{c}\left(X_{11}-q^{2} X_{32}\right)$ \\
$u_{3}^{C}$ & $(1-\alpha)\left(\tilde{y} X_{11}+\xi Y_{11} \cos \delta\right)+\alpha q\left(\tilde{c} \eta X_{32}+\xi Z_{32}\right)$ \\
\hline$j_{1}^{C}$ & X-derivative \\
$j_{2}^{C}$ & $(1-\alpha) \xi \sin \delta / R^{3}+\xi q Y_{32} \cos \delta+\alpha \eta\left(3 \tilde{c} \eta / R^{5}-2 Z_{32}-Z_{0}\right)$ \\
$j_{3}^{C}$ & $-(1-\alpha) 2 Y_{0} \sin \delta-\tilde{d} / R^{3}+\alpha \tilde{c}\left(1-3 q^{2} / R^{2}\right) / R^{3}$ \\
\hline & $(1-\alpha)\left(q / R^{3}+Y_{0} \sin \delta \cos \delta\right)-\alpha\left(3 \tilde{c} \eta q / R^{5}-q Z_{0}\right)$ \\
\hline$k_{1}^{C}$ & $-(1-\alpha) 2 \xi P \sin \delta-\tilde{y} \tilde{d} X_{32}+\alpha \tilde{c}\left[(\tilde{d}+2 q \sin \delta) X_{32}-\tilde{y} q^{2} X_{53}\right]$ \\
$k_{2}^{C}$ & $-(1-\alpha)\left(\xi P \cos \delta-X_{11}+\tilde{y}^{2} X_{32}\right)+\alpha \tilde{c}\left[(\tilde{d}+2 q \cos \delta) X_{32}-\tilde{y} \eta q X_{53}\right]+\alpha \xi Q$ \\
$k_{3}^{C}$ &
\end{tabular}

Table 29. Scaling (internal deformation and strain; function $f_{C}$ ).

[Okada (1992), $\mathrm{i}=1$ : strike-slip; $\mathrm{i}=2$ : dip-slip; $\mathrm{i}=3$ tensile e-for displacement, see table 6; for X-derivative, see table 7, and for Y-derivative, see table 8]

\begin{tabular}{cc}
\hline & Displacement \\
\hline$u_{1}^{C}$ & $\left(0.5 U_{i} / \pi\right) u_{1}^{C}$ \\
$u_{2}^{C}$ & $\left(0.5 U_{i} / \pi\right) u_{2}^{C}$ \\
$u_{3}^{C}$ & $\left(0.5 U_{i} / \pi\right) u_{3}^{C}$ \\
\hline & X-derivative \\
\hline$j_{1}^{C}$ & $\left(0.5 U_{i} / \pi\right) j_{1}^{C}$ \\
$j_{2}^{C}$ & $\left(0.5 U_{i} / \pi\right) j_{2}^{C}$ \\
$j_{3}^{C}$ & $\left(0.5 U_{i} / \pi\right) j_{3}^{C}$ \\
\hline & Y-derivative \\
\hline$k_{1}^{C}$ & $\left(0.5 U_{i} / \pi\right) k_{1}^{C}$ \\
$k_{2}^{C}$ & $\left(0.5 U_{i} / \pi\right) k_{2}^{C}$ \\
$k_{3}^{C}$ & $\left(0.5 U_{i} / \pi\right) k_{3}^{C}$ \\
\hline
\end{tabular}




\section{Verification and Examples}

\section{Strike-Slip Fault (see fig. 33 and table 30)}
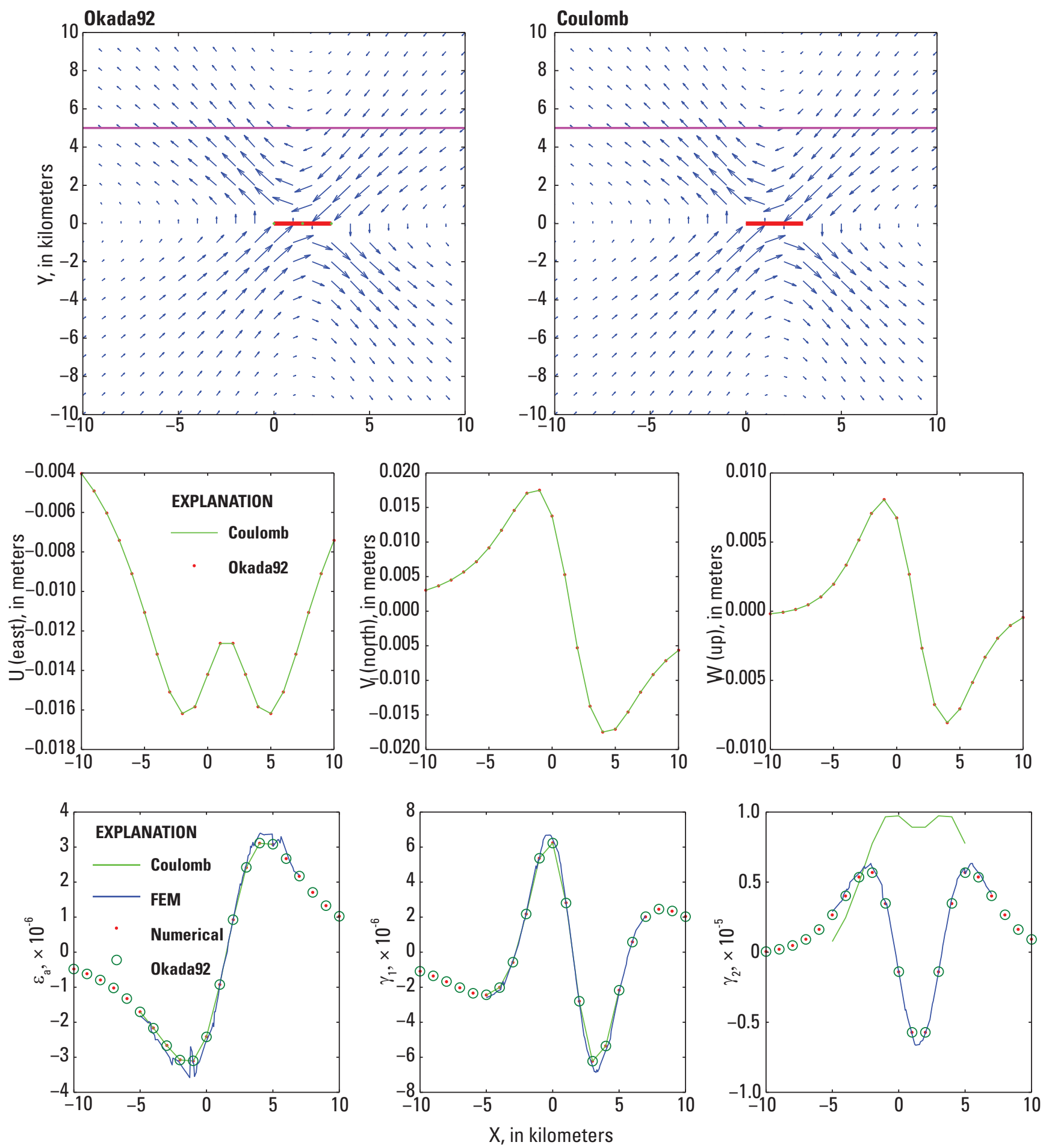

Figure 33. Vector (top) and profile plots showing displacement field (center) and strain (bottom) caused by a strike-slip fault (thick red line). The profile was taken at a depth of 1 kilometer along the magenta line (top). The difference between the $0 \mathrm{kada92}$ solution for $\gamma_{2}$ and that from COULOMB 3.3 is due to an error in COULOMB 3.3 (R. Stein, U.S. Geological Survey, oral commun.). Okada92, Matlab script of Okada's (1992) expressions; Coulomb 3.3, solution from COULOMB 3.3; FEM, numerical solution from a finite element method numerical model; numerical, strain from finite-difference derivatives (appendix 1). Fault parameters are $x_{i}=0$ meters, $y_{i}=0$ meters, $x_{f}=3,000$ meters, $y_{f}=0$ meters, $\delta=90^{\circ}, \mu=1$ Gigapascals, $v=0.25, U=1$ meter, $z_{t}=2,000$ meters, and $z_{b}=$ 4,000 meters. See table 30 for numerical example. 
Table 30. Internal deformation and strain around a strike-slip fault.

[See also figure 33. Parameters are $x_{i}=0$ meters, $y_{i}=0$ meters, $x_{f}=3,000$ meters; $y_{f}=0$ meters; $z_{t}=2,000$ meters; $z_{b}=4,000$ meters; $x, y$ and $z$, local Cartesian coordinates; $u_{x}, u_{y}$ and $u_{z}$, internal deformation; $\varepsilon_{a}, \gamma_{1}$ and $\gamma_{2}$, strain; $\mu$ strain , a strain expressed in terms of parts per million]

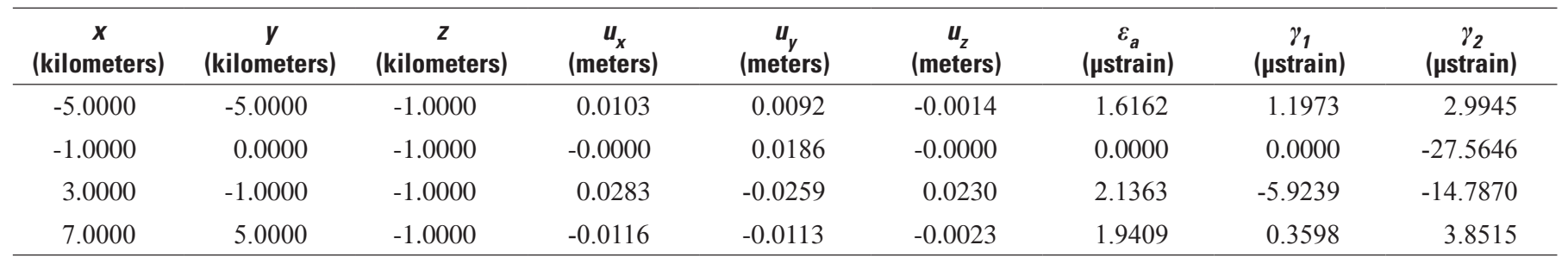




\section{Dip-Slip Fault (see fig. 34 and table 31)}
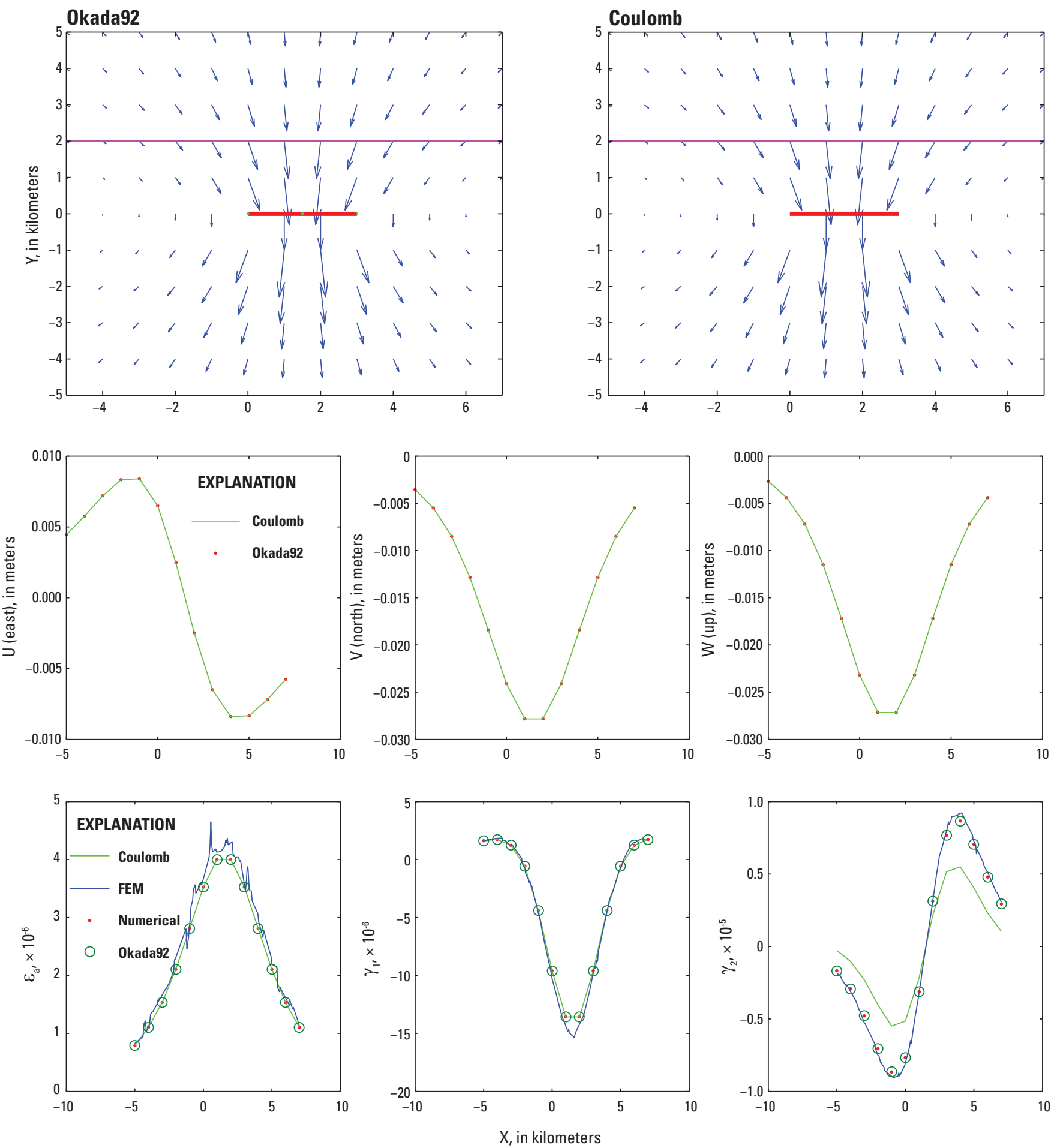

Figure 34. Vector (top) and profile plots of the displacement field (center) and strain (bottom) caused by a dip-slip fault (thick red line). The profile was taken at a depth of 1 kilometer along the magenta line (top). The difference between the 0 kada92 solution for $\gamma_{2}$ and that from COULOMB 3.3 is due to a error in COULOMB 3.3 (R. Stein, U.S. Geological Survey, oral commun.). Okada92, Matlab script of Okada's (1992) expressions; Coulomb 3.3, solution from COULOMB 3.3; FEM, numerical solution from FEM model; numerical, strain from finite-difference derivatives (appendix 1). Fault parameters: $x_{i}=0$ meters, $y_{i}=0$ meters, $x_{f}=3,000$ meters; $y_{\mathrm{f}}=0$ meters; $\delta=90^{\circ} ; \mu=1 \mathrm{GPa} ; v=0.25 ; U=1$ meters; $z_{\mathrm{t}}=2,000$ meters; $z_{\mathrm{b}}=4,000$ meters. 
Table 31. Internal deformation and strain around a dip-slip fault.

[See figure 34. Parameters are $x_{i}=0$ meters, $y_{i}=0$ meters, $x_{f}=3,000$ meters; $y_{\mathrm{f}}=0$ meters; $\delta=90^{\circ} ; \mu=1 \mathrm{GPa} ; v=0.25 ; \mathrm{U}=1 \mathrm{~meters} ; z_{t}=2,000$ meters; $z_{b}=$ 4,000 meters; $x, y$ and $z$, local Cartesian coordinates; $u_{x}, u_{y}$ and $u_{z}$, internal deformation; $\varepsilon_{a}, \gamma_{1}$ and $\gamma_{2}$, strain; $\mu$ strain, a strain expressed in terms of parts per million]

\begin{tabular}{|c|c|c|c|c|c|c|c|c|}
\hline $\begin{array}{c}x \\
\text { (kilometers) }\end{array}$ & $\begin{array}{c}y \\
\text { (kilometers) }\end{array}$ & $\begin{array}{c}z \\
\text { (kilometers ) }\end{array}$ & $\begin{array}{c}u_{x} \\
\text { (meters) }\end{array}$ & $\begin{array}{c}u_{y} \\
\text { (meters) }\end{array}$ & $\begin{array}{c}u_{z} \\
\text { (meters) }\end{array}$ & $\begin{array}{c}\varepsilon a \\
\text { ( } \mu \text { strain) }\end{array}$ & $\begin{array}{c}\gamma_{1} \\
\text { (ustrain) }\end{array}$ & $\begin{array}{c}\gamma_{2} \\
\text { ( } \mu \text { strain) }\end{array}$ \\
\hline-5.0000 & -5.0000 & -1.0000 & 0.0041 & -0.0038 & 0.0024 & -0.7843 & -1.0461 & -1.9073 \\
\hline 3.0000 & -1.0000 & -1.0000 & 0.0177 & -0.0486 & 0.0653 & 5.7981 & 0.4789 & 26.5485 \\
\hline 7.0000 & 5.0000 & -1.0000 & 0.0051 & -0.0056 & -0.0036 & 1.0556 & 0.8267 & 2.9135 \\
\hline
\end{tabular}




\section{Tensile Crack (see fig. 35 and table 32)}
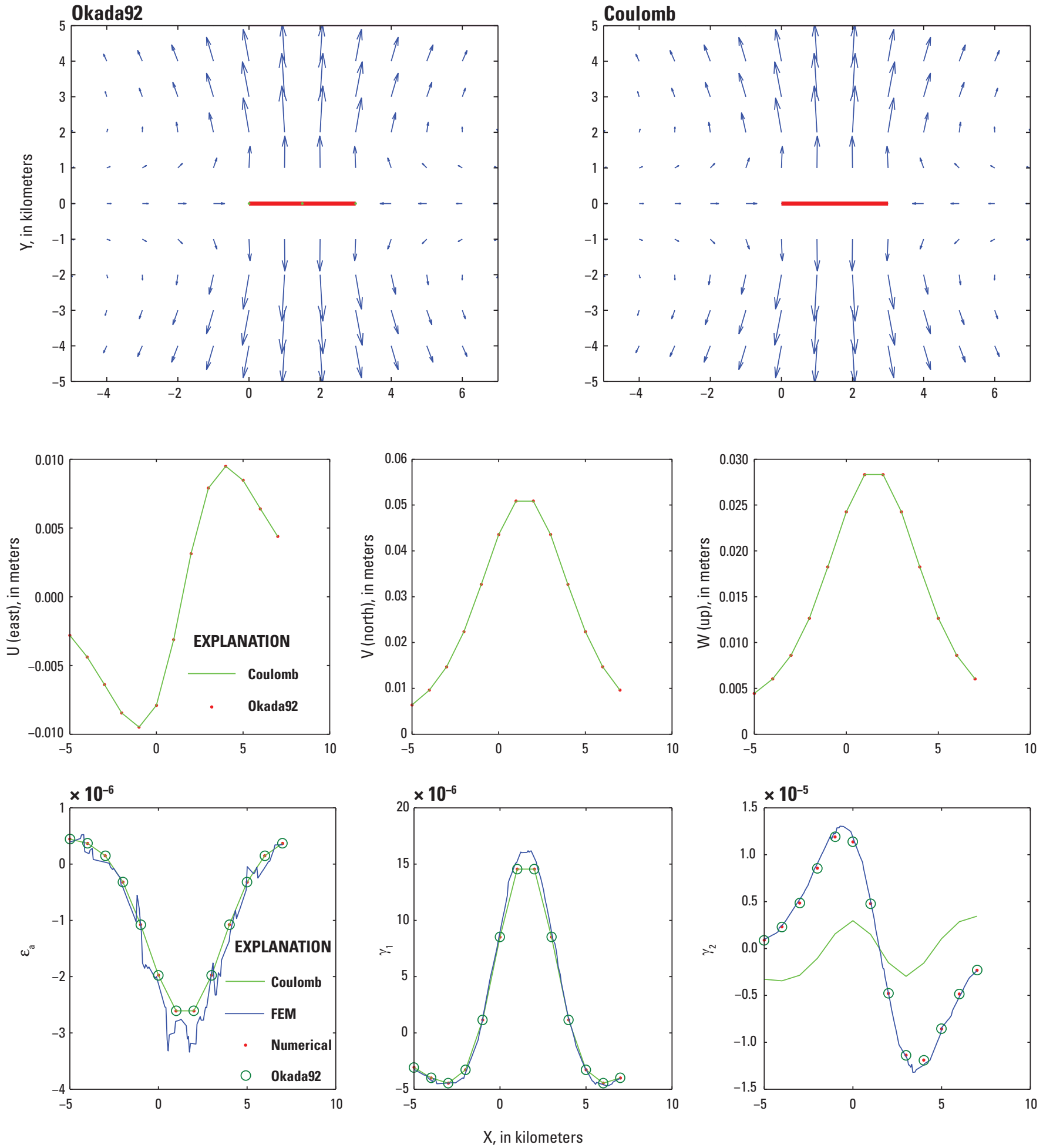

Figure 35. Vector (top) and profile plots of the displacement field (center) and strain (bottom) caused by a tensile crack (thick red line). The profile was taken at a depth of 1 kilometer along the magenta line (top). The difference between the Okada92 solution for $\gamma_{2}$ and that from COULOMB 3.3 is due to a error in COULOMB 3.3 (R. Stein, U.S. Geological Survey, oral commun.). Okada92, Matlab script of Okada's (1992) expressions; Coulomb 3.3, solution from COULOMB 3.3; FEM, numerical solution from FEM model; numerical, strain from finite-difference derivatives (appendix 1). Fault parameters: $x_{\mathrm{i}}=0$ meters, $y_{\mathrm{i}}=0$ meters, $x_{f}=3,000$ meters; $y_{f}=0$ meters; $\delta=90^{\circ} ; \mu=1 \mathrm{GPa} ; v=0.25 ; \mathrm{U}=1 \mathrm{~m} ; z_{t}=2,000$ meters; $z_{b}=4,000$ meters. 
Table 32. Internal deformation and strain around a tensile crack.

[See figure 35. Parameters are $x_{i}=0$ meters, $y_{i}=0$ meters, $x_{f}=3,000$ meters; $y_{f}=0$ meters; $\delta=90^{\circ} ; \mu=1$ Gigapascal; $v=0.25, U=1$ meter, $z_{t}=2,000$ meters; $z_{b}=4,000$ meters. $x, y$ and $z$, local Cartesian coordinates; $u_{x}, u_{y}$ and $u_{z}$, internal deformation; $\varepsilon_{a}, \gamma_{1}$ and $\gamma_{2}$, strain; $\mu$ strain, a strain expressed in terms of parts per million]

\begin{tabular}{|c|c|c|c|c|c|c|c|c|}
\hline $\begin{array}{c}\mathrm{x} \\
\text { (kilometers) }\end{array}$ & $\begin{array}{c}y \\
\text { (kilometers) }\end{array}$ & $\begin{array}{c}z \\
\text { (kilometers) }\end{array}$ & $\underset{\text { (meters) }}{u_{x}}$ & $\begin{array}{c}u_{y} \\
\text { (meters) }\end{array}$ & $\underset{\text { (meters) }}{u_{z}}$ & $\begin{array}{c}\varepsilon a \\
\text { (pstrain) }\end{array}$ & $\begin{array}{c}\gamma_{1} \\
\text { (pstrain) }\end{array}$ & $\underset{\text { (ustrain) }}{\gamma_{2}}$ \\
\hline-5.0000 & -5.0000 & -1.0000 & -0.0041 & -0.0079 & 0.0041 & -0.0138 & -2.6669 & -1.9129 \\
\hline-1.0000 & 0.0000 & -1.0000 & 0.0152 & -0.0000 & -0.0058 & 14.8157 & -7.2473 & -0.0000 \\
\hline 3.0000 & -1.0000 & -1.0000 & -0.0013 & -0.0296 & 0.0141 & 27.4109 & -32.0105 & 2.6374 \\
\hline 7.0000 & 5.0000 & -1.0000 & 0.0056 & 0.0114 & 0.0055 & -0.2245 & -2.9155 & -3.4452 \\
\hline
\end{tabular}




\section{Discretized Faults}

Inverse modeling of surface-displacement measurements is required to estimate spatial slip variations along the faults that ruptured in an earthquake. Appropriate analytical solutions can relate surface displacements to offsets across many small rectangular dislocations (referred to as segments, subsegments, and tiles ${ }^{4}$ in the model; see fig. 36) that may or may not slip in a halfspace that is homogeneous, isotropic, flat, and elastic.

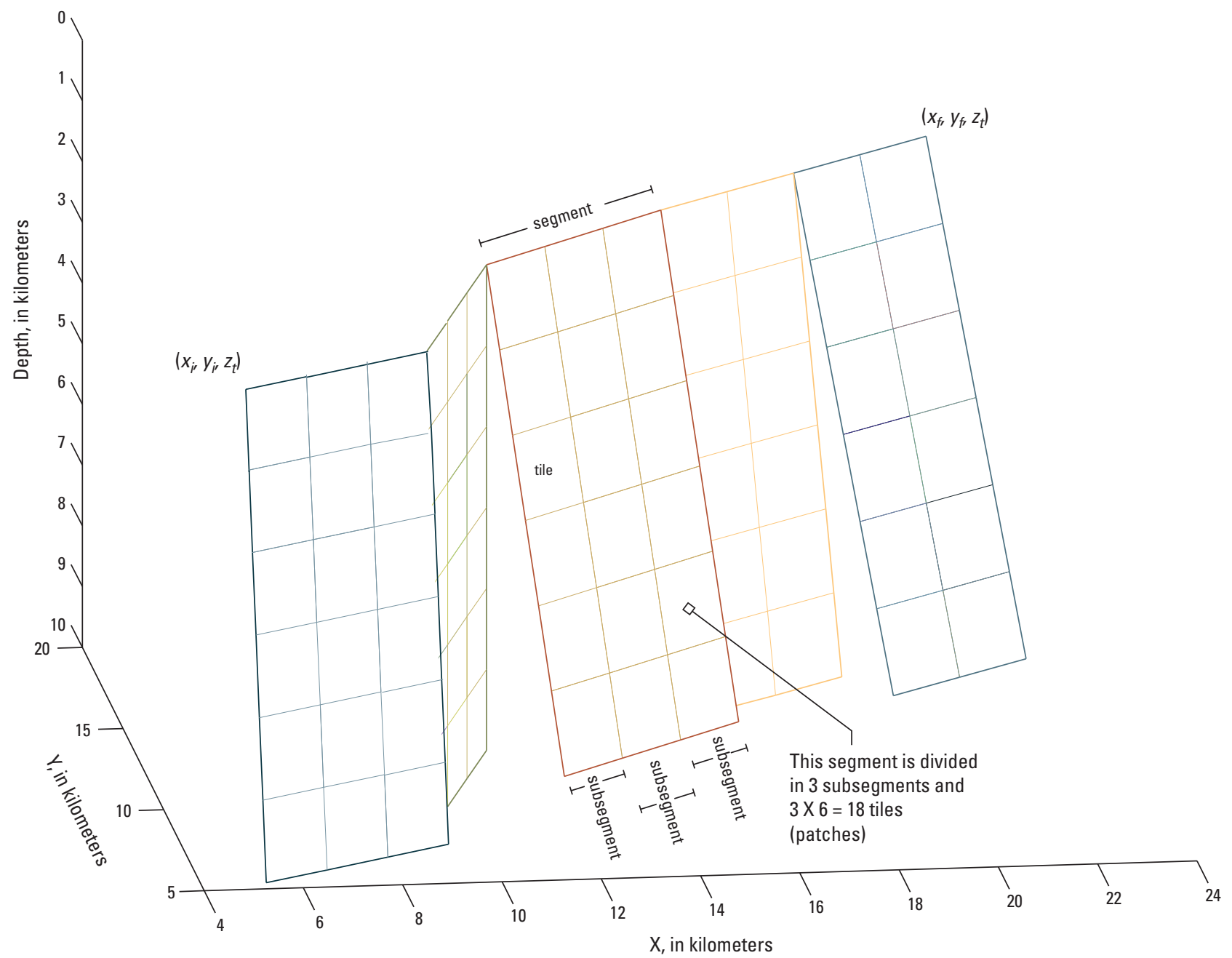

Figure 36. Representation of the fault geometry by segments, subsegments, and tiles. The fault above has 5 segments, 13 subsegments and 78 tiles. The fault is identified by its initial $\left(x_{i}, y_{i}, z_{t}\right)$ and final $\left(x_{f}, y_{f}, z_{t}\right)$ coordinates; $z_{t}$, depth of the top edge of the fault. 
The chart below (fig. 37) describes the process for coding a discretized fault.

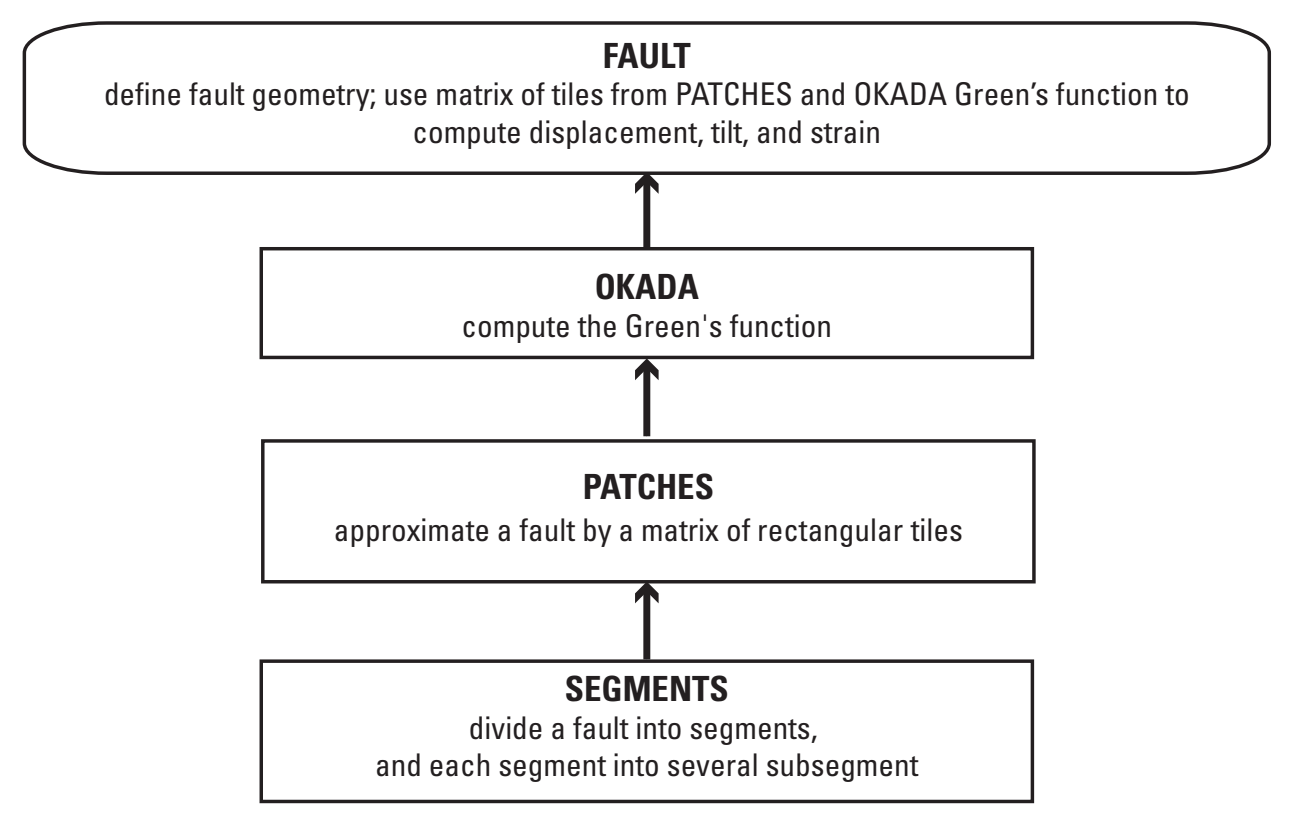

Figure 37. The Matlab script for coding a discretized fault includes four major steps: (1) the function SEGMENTS divides a fault into several rectangular dislocations (segments and subsegments); (2) each segment/subsegment is divided into several tiles by the function PATCHES, approximating the fault with a matrix of tiles; (3) the function FAULT uses the function OKADA to compute the displacement of each tile, and then (4) add the displacements of all of the tiles to compute the crustal deformation caused by the slip along the fault. 


\section{Fault Segments and Subsegments}

Estimating the location, size, dip, orientation, and slip across multiple rectangular dislocations that may or may not slip is a numerically challenging task given the number of data points and the number of parameters required to estimate these dislocations. Information from the mapped fault trace can be used to constrain several parameters of the fault geometry, including the number of segments that could reasonably represent the fault trace at the surface:

$$
L=\sum_{n=1}^{N} \sqrt{\left.\left(x_{n}^{i}-x_{n}^{f}\right)\right)^{2}+\left(y_{n}^{i}-y_{n}^{f}\right)^{2}}
$$

and

$$
N=\left\lceil\frac{\text { length of fault trace }}{\text { width of segment }}\right\rceil,
$$

where

$\left(x_{n}^{i}, y_{n}^{i}\right)$ and

$L \quad$ is the length of the fault trace,

$\left(x_{n}^{f}, y_{n}^{f}\right)$

are the initial and final points of the segment,

$n \quad$ is index of the $n^{\text {th }}$ segment,

$\mathrm{N}$ is the number of segments, and

\lceil\rceil is the ceiling function (fig. 36). The $(x, y)$ coordinates of points of the grid along the upper edge of each subsegment (figs. 36 and 38) are defined by

$$
\begin{aligned}
& x x=x_{n}^{i}+(0: 1: S) \frac{x_{n}^{f}-x_{n}^{i}}{S} \\
& y y=y_{n}^{f}+\left(y_{n}^{f}-y_{n}^{i}\right) \frac{x x-x_{n}^{f}}{x_{n}^{f}-x_{n}^{i}}
\end{aligned}
$$

where $S$ is the total number of subsegments, and each subsegment's initial and final point

$$
\begin{aligned}
& x_{s}^{i}=x x(1: S), \quad y_{s}^{i}=y y(1: S) \\
& x_{s}^{f}=x x(2: S+1), \quad y_{s}^{f}=y y(2: S+1)
\end{aligned}
$$




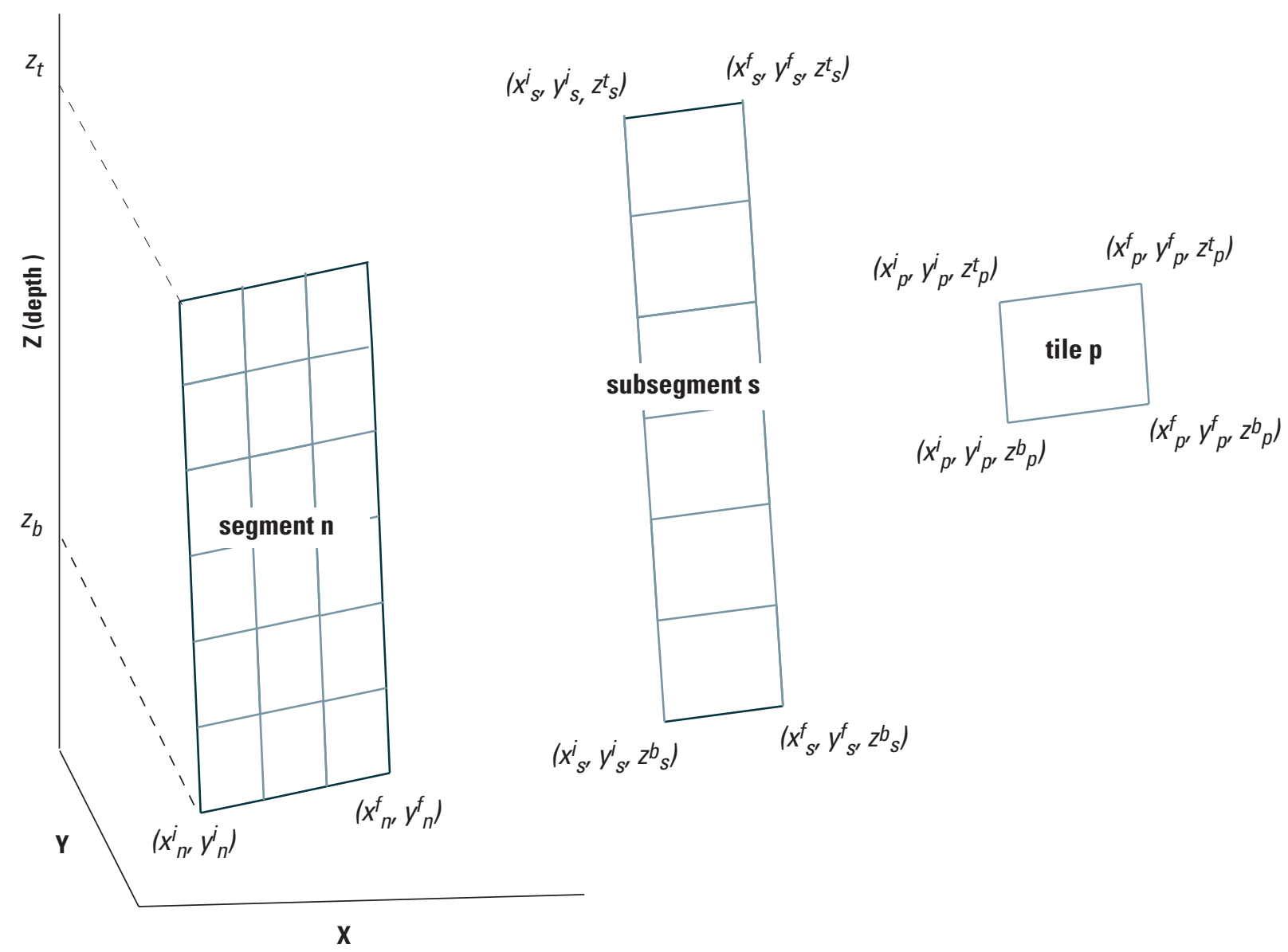

Figure 38. Segment, subsegment, and tile geometry. $\left(x^{i}, y^{i}\right)$, horizontal coordinates for the left corner of segments, subsegments, or tiles; $\left(x^{f}, y^{f}\right)$, horizontal coordinates for the right corner segments, subsegments, or tiles; $z_{t}$, depth of the top edge of segments, subsegments or tiles; $z_{b}$, depth of the bottom edge of segments, subsegments, or tiles; $n, s$ or $p$, subindex for segments, subsegments, or tiles.

\section{Tiles}

Each subsegment (figs. 36 and 38) can be divided into several tiles following the steps described below. First, define three parameters:

1. the subsegment at the initial (lower left) corner

$$
\begin{aligned}
& x_{0}^{i}=x_{s}^{i}+\left(z_{s}^{b}-z_{s}^{t}\right) \cot \delta \cos \phi \\
& y_{0}^{i}=y_{s}^{i}-\left(z_{s}^{b}-z_{s}^{t}\right) \cot \delta \sin \phi
\end{aligned}
$$


2. the subsegment at the final (lower right) corner

$$
\begin{aligned}
& x_{0}^{f}=x_{s}^{f}+\left(z_{s}^{b}-z_{s}^{t}\right) \cot \delta \cos \phi, \text { and } \\
& y_{0}^{f}=y_{s}^{f}-\left(z_{s}^{b}-z_{s}^{t}\right) \cot \delta \sin \phi
\end{aligned}
$$

3. the number of tiles $P$ for each subsegment (the symbol \lceil\rceil is the ceiling function)

$$
P=\left\lceil\frac{\text { height of subsegment }}{\text { height of tile }}\right\rceil \text {. }
$$

Then, compute the coordinates of the grid of tiles at the initial (left) and final (right) side of each segment by using the formulas below:

1. grid along the depth

$$
z z=z_{s}^{t}+(0: 1: P)\left(z_{s}^{b}-z_{s}^{t}\right) / P
$$

2. grid along the left side of the segment

$$
\begin{aligned}
& x x_{i}=x_{0}^{i}+\left(x_{s}^{i}-x_{0}^{i}\right)\left(z z-z_{s}^{b}\right) /\left(z_{s}^{t}-z_{s}^{b}\right) \text { and } \\
& y y_{i}=y_{0}^{i}+\left(y_{s}^{i}-y_{0}^{i}\right)\left(z z-z_{s}^{b}\right) /\left(z_{s}^{t}-z_{s}^{b}\right)
\end{aligned}
$$

3. grid along the right side of the segment

$$
\begin{aligned}
& x x_{f}=x_{0}^{f}+\left(x_{s}^{f}-x_{0}^{f}\right)\left(z z-z_{s}^{b}\right) /\left(z_{s}^{t}-z_{s}^{b}\right) . \\
& y y_{f}=y_{0}^{f}+\left(y_{s}^{f}-y_{0}^{f}\right)\left(z z-z_{s}^{b}\right) /\left(z_{s}^{t}-z_{s}^{b}\right)
\end{aligned}
$$

Finally, we define the tile at the top left corner (initial point), the tile at the top right corner (final point), and the tiles at the top and bottom as

$$
\begin{aligned}
x_{p}^{i} & =x x_{i}(1: P) & y_{p}^{i} & =y y_{i}(1: P) \\
x_{p}^{f} & =x x_{f}(1: P) & y_{p}^{f} & =y y_{f}(1: P) \\
z_{p}^{t} & =z z(1: P) & z_{p}^{b} & =z z(2: P+1)
\end{aligned}
$$

\section{Verification of the Discretized Fault Model}

We approximate a fault by a matrix of rectangular tiles. The model computes the deformation of a single tile by using Green's function for a rectangular dislocation (Okada, 1985, 1992) and then adds the contributions to calculate the total deformation (see tables 33, 34 and 35 for numerical examples). We compared our Matlab coding of the analytical expressions for discretized strike-slip faults (fig. 39), dip-slip faults (fig. 40), and tensile cracks (fig. 41) faults against solutions from COULOMB 3.3. 


\section{Strike-Slip Fault}
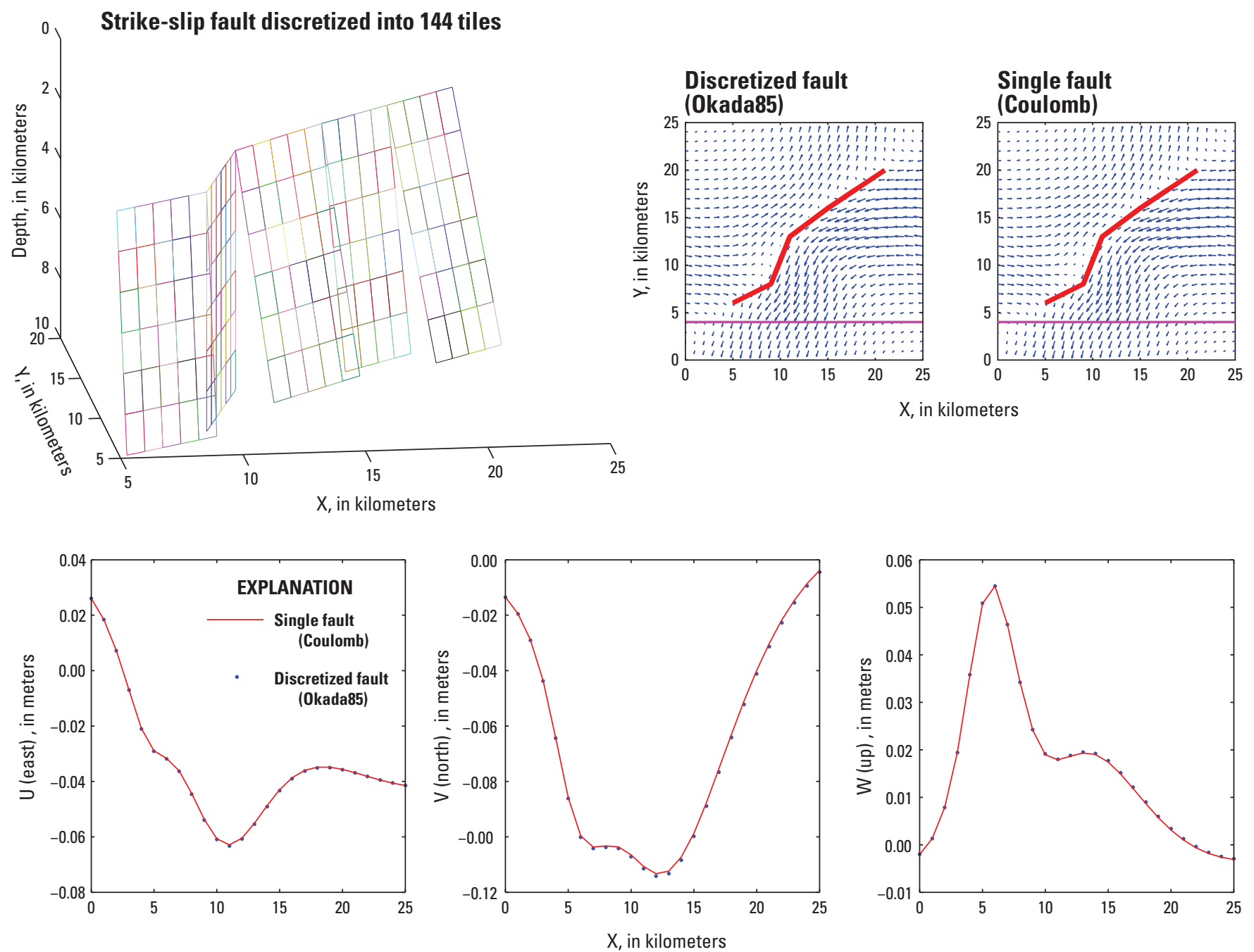

Figure 39. Fault geometry and vector plot (top row) and profile plots (bottom row) of the surface displacement caused by a rightlateral strike-slip fault (thick red line). The profile is along the magenta line (top row, right). Top row—0kada85, Matlab script of Okada's (1985) expressions; COULOMB, solution from COULOMB 3.3. Bottom row- "Discretized fault" labels the solution obtained by applying the Okada (1985) equations to a fault composed of 144 tiles (top row, left). "Single fault" labels the solution from COULOMB 3.3 for a fault divided into five segments, each of which exhibited uniform slip. See table 33 for numerical example.

Table 33. Parameters for strike-slip fault segments.

[Parameters given here are for right-lateral strike-slip fault segments. See figure 39. $x_{\mathrm{i}}, y_{i}, x_{p}$ and $y_{f}$ coordinates of the initial and final positions along the top edges of the fault segments; $U_{1}$, final right-lateral dislocation of segment; $z_{t}$ and $z_{b}$, coordinates of the top and bottom of the segment]

\begin{tabular}{ccccccccc}
\hline Segment & $\begin{array}{c}\boldsymbol{x}_{\boldsymbol{i}} \\
\text { (kilometers) }\end{array}$ & $\begin{array}{c}\boldsymbol{y}_{\boldsymbol{i}} \\
\text { (kilometers) }\end{array}$ & $\begin{array}{c}\boldsymbol{x}_{\boldsymbol{f}} \\
\text { (kilometers) }\end{array}$ & $\begin{array}{c}\boldsymbol{y}_{\boldsymbol{f}} \\
\text { (kilometers) }\end{array}$ & $\begin{array}{c}\boldsymbol{U}_{\boldsymbol{1}} \\
\text { (meters) }\end{array}$ & $\begin{array}{c}\text { Dip angle } \\
\text { (degrees) }\end{array}$ & $\begin{array}{c}\boldsymbol{z}_{\boldsymbol{t}} \\
\text { (kilometers) }\end{array}$ & $\begin{array}{c}\boldsymbol{z}_{\mathrm{b}} \\
\text { (kilometers) }\end{array}$ \\
\hline 1 & 5 & 6 & 9 & 8 & 1 & 85 & 2 & 10 \\
2 & 9 & 8 & 11 & 13 & 0.5 & 90 & 2 & 10 \\
3 & 11 & 13 & 15 & 16 & 1.5 & 75 & 2 & 10 \\
4 & 15 & 16 & 18 & 18 & 0.5 & 80 & 2 & 10 \\
5 & 18 & 18 & 21 & 20 & 0.75 & 70 & 2 & 10 \\
\hline
\end{tabular}




\section{Dip-Slip Fault}
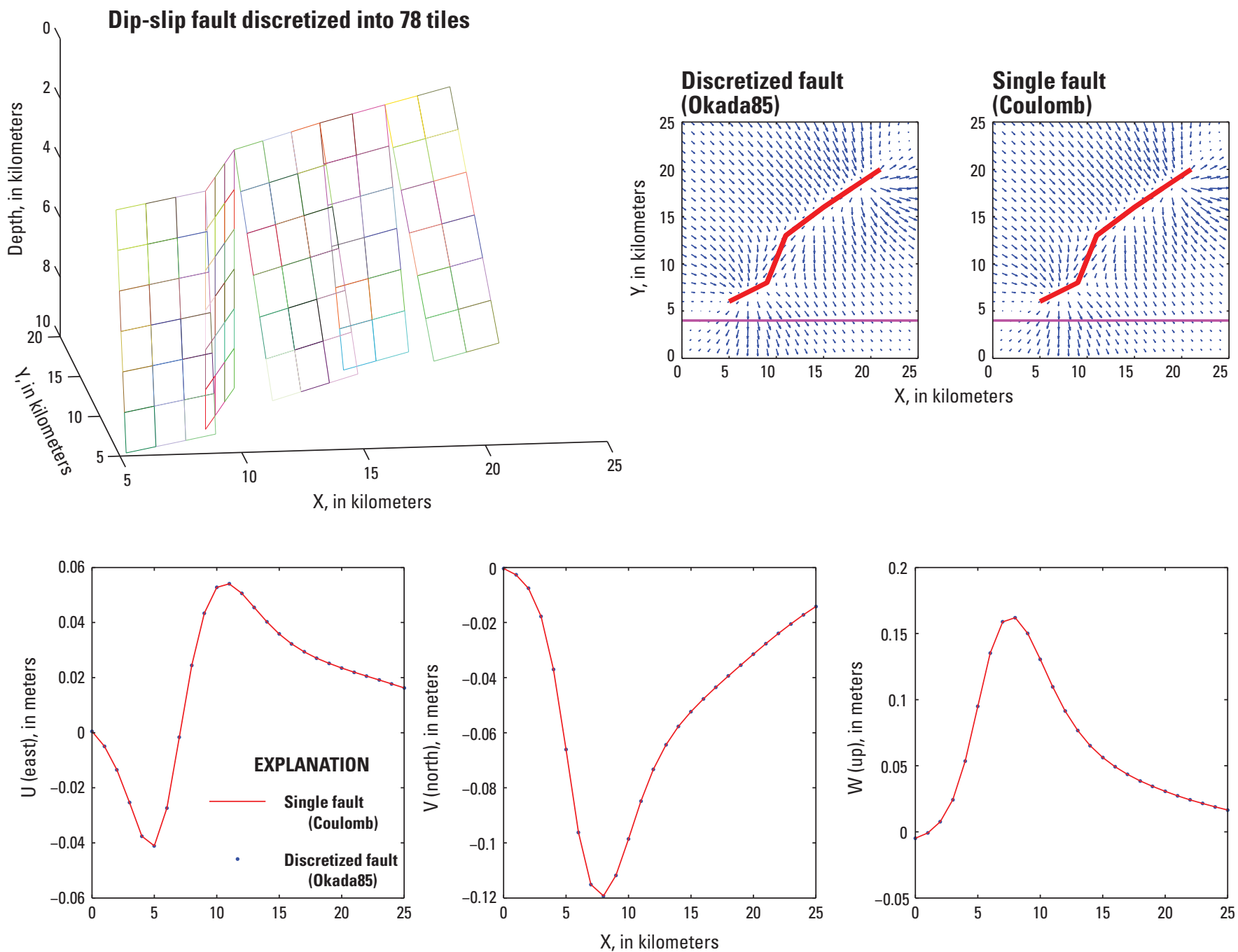

Figure 40. Fault geometry and vector plot (top row) and profile plots (bottom row) of the surface displacement caused by a reverse dip-slip fault. The profile is along the magenta line (top row, right). Top row-Okada85, Matlab script of Okada's (1985) expressions; COULOMB, solution from COULOMB 3.3. Bottom row-“"Discretized fault" labels the solution obtained by applying the Okada (1985) equations to a fault composed of 78 tiles (top row, left). "Single fault" labels the solution from COULOMB 3.3 for a fault divided into five segments, each of which exhibited uniform slip. See table 34 for numerical example.

Table 34. Parameters for dip-slip fault segments.

[Parameters given are for reverse dip-slip fault segments. See figure 40. $x_{\mathrm{i}}, y_{i}, x_{f}$ and $y_{f}$, coordinates of the initial and final positions along the top edges of the fault segments; $U_{2}$, final reverse dislocation of segment; $z_{t}$ and $z_{b}$, coordinates of the top and bottom of the segment.

\begin{tabular}{ccccccccc}
\hline Segment & $\begin{array}{c}\boldsymbol{x}_{\boldsymbol{i}} \\
\text { (kilometers) }\end{array}$ & $\begin{array}{c}\boldsymbol{y}_{\boldsymbol{i}} \\
\text { (kilometers) }\end{array}$ & $\begin{array}{c}\boldsymbol{x}_{\boldsymbol{f}} \\
\text { (kilometers) }\end{array}$ & $\begin{array}{c}\boldsymbol{y}_{\boldsymbol{f}} \\
\text { (kilometers) }\end{array}$ & $\begin{array}{c}\boldsymbol{U}_{\mathbf{2}} \\
\text { (meters) }\end{array}$ & $\begin{array}{c}\text { Dip angle } \\
\text { (degrees) }\end{array}$ & $\begin{array}{c}\boldsymbol{z}_{\boldsymbol{t}} \\
\text { (kilometers) }\end{array}$ & $\begin{array}{c}\boldsymbol{z}_{\boldsymbol{b}} \\
\text { (kilometers) }\end{array}$ \\
\hline 1 & 5 & 6 & 9 & 8 & 1 & 85 & 2 & 10 \\
2 & 9 & 8 & 11 & 13 & 0.1 & 90 & 2 & 10 \\
3 & 11 & 13 & 15 & 16 & 0.7 & 75 & 2 & 10 \\
4 & 15 & 16 & 18 & 18 & 0.5 & 80 & 2 & 10 \\
5 & 18 & 18 & 21 & 20 & 1.5 & 70 & 2 & 10 \\
\hline
\end{tabular}




\section{Tensile Crack}
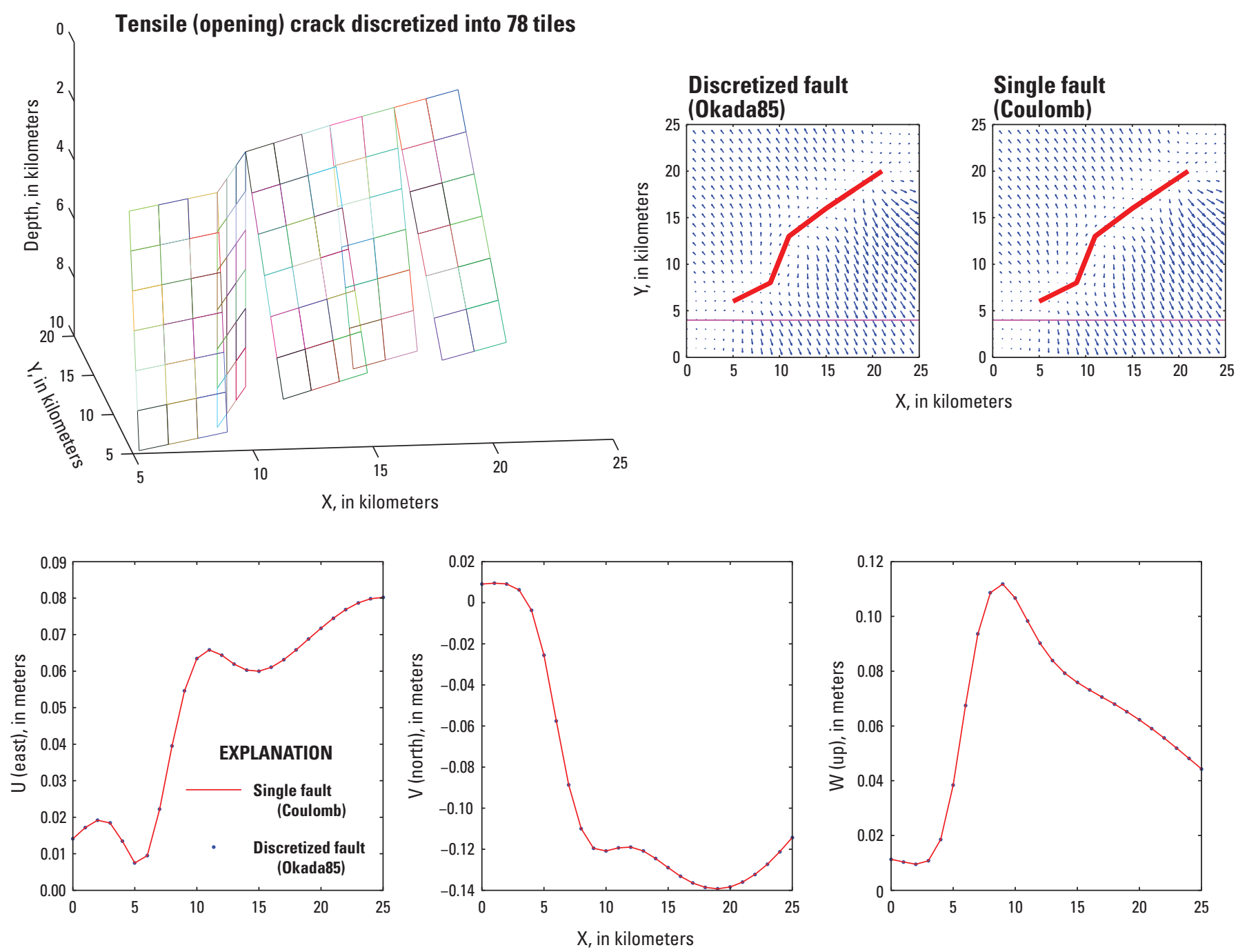

Figure 41. Fault geometry and vector plot (top row) and profile plots (bottom row) of the surface displacement caused by the opening of a tensile crack. The profile is along the magenta line (top row, right). Top row-0kada85, Matlab script of 0kada's (1985) expressions; COULOMB, solution from COULOMB 3.3. Bottom row-“Discretized fault” labels the solution obtained by applying the 0kada (1985) equations to a fault composed of 78 tiles (top row, left). "Single fault" labels the solution from COULOMB 3.3 for a fault divided into five segments, each of which exhibited uniform slip. See table 35 for numerical example.

Table 35. Parameters for tensile-crack fault segments.

[Parameters given are for the tensile-crack shown in figure 41. $x_{\mathrm{i}}, y_{i}, x_{f}$ and $y_{f}$, coordinates of the initial and final positions along the top edges of the fault segments; $U_{3}$, final opening dislocation of segment; $z_{t}$ and $z_{b}$, coordinates of the top and bottom of the segment]

\begin{tabular}{ccccccccc}
\hline Segment & $\begin{array}{c}\boldsymbol{x}_{\boldsymbol{i}} \\
\text { (kilometers) }\end{array}$ & $\begin{array}{c}\boldsymbol{y}_{\boldsymbol{i}} \\
\text { (kilometers) }\end{array}$ & $\begin{array}{c}\boldsymbol{x}_{\boldsymbol{f}} \\
\text { (kilometers) }\end{array}$ & $\begin{array}{c}\boldsymbol{y}_{\boldsymbol{f}} \\
\text { (kilometers) }\end{array}$ & $\begin{array}{c}\boldsymbol{U}_{3} \\
\text { (meters) }\end{array}$ & $\begin{array}{c}\text { Dip angle } \\
\text { (degrees) }\end{array}$ & $\begin{array}{c}\boldsymbol{z}_{\boldsymbol{t}} \\
\text { (kilometers) }\end{array}$ & $\begin{array}{c}\boldsymbol{z}_{\boldsymbol{b}} \\
\text { (kilometers) }\end{array}$ \\
\hline 1 & 5 & 6 & 9 & 8 & 1 & 85 & 2 & 10 \\
2 & 9 & 8 & 11 & 13 & 0.1 & 90 & 2 & 10 \\
3 & 11 & 13 & 15 & 16 & 0.7 & 75 & 2 & 10 \\
4 & 15 & 16 & 18 & 18 & 0.5 & 80 & 2 & 10 \\
5 & 18 & 18 & 21 & 20 & 1.5 & 70 & 2 & 10 \\
\hline
\end{tabular}




\section{Smoothing Operator D}

The surface deformation can be inverted onto fault planes that are discretized with evenly sized dislocations; fault tiles in the model may or may not slip (see Jonsson and others, 2002; Barnhart and Lohman, 2010). The observed surface displacements $\mathbf{d}$ can be described as a function $g(\mathbf{m})$ of the fault model parameters $\mathbf{m}$ :

$$
\mathbf{d}=g(\mathbf{m})
$$

where the function $g$ describes how slip across the rectangular dislocations causes displacement at the ground surface (Okada, 1985). The surface displacements are nonlinear functions of the fault- geometry parameters (for example, length, width, location, dip, and strike) and linear functions of the slip along the fault

$$
g(\mathbf{m})=\mathbf{G s}
$$

where $\mathbf{G}$ is a matrix of data kernels (for example, Green's functions from Okada, 1985), which depend on the assumed fault-geometry parameters and relate the surface displacement to the model; and $\mathbf{S}$ is the vector of slip values for every tile. By substituting equation 139 into equation 138, the surface displacement $\mathbf{d}$ as a linear system in matrix form

$$
\mathbf{d}=\mathbf{G s} \text {. }
$$

Finally, the displacement $\mathbf{d}$ is weighted by using the data covariance matrix $\Sigma$

$$
\mathbf{W d}=\mathbf{W G s} \quad \text { and } \quad \mathbf{W}^{\mathbf{T}} \mathbf{W}=\Sigma^{-1}
$$

where $\mathbf{W}$ is the weight matrix from the Cholesky decomposition of the data covariance matrix.

The system of equations relating slip on each tile to the surface displacement $\mathbf{d}$ consists of both a matrix of Green's functions $\mathbf{G}$ and a smoothing matrix $\mathbf{D}$

$$
\left[\begin{array}{c}
\mathbf{W d} \\
\mathbf{0}
\end{array}\right]=\left[\begin{array}{c}
\mathbf{W G} \\
\kappa^{2} \mathbf{D}
\end{array}\right] \mathbf{s},
$$

The smoothness constraint on the slip $\mathbf{S}$ minimizes the two-dimensional second derivative (Laplacian) of the fault slip. The Lagrange multiplier $\kappa^{2}$ determines the weight of smoothing, and $\mathbf{D}$ is the second-order finite difference operator

$$
\nabla^{2} \mathbf{s}=\mathbf{D s}
$$

\section{Building the Smoothing Operator}

We can build the smoothing operator $\mathbf{D}$ for a nonuniform grid in three steps:

1. Compute the location of the center of each tile.

2. Compute the vertical and horizontal distances between neighboring tile centers assuming that the vertical distances $\left(\Delta h_{2}\right.$ and $\left.\Delta h_{4}\right)$ are constant (all the rows of the discretized fault are of equal width), whereas the horizontal distances $\left(\Delta h_{1}\right.$ and $\left.\Delta h_{3}\right)$ may vary (the width of the fault columns can change), figure 42 .

3. Compute the $\mathbf{D}$ matrix. 


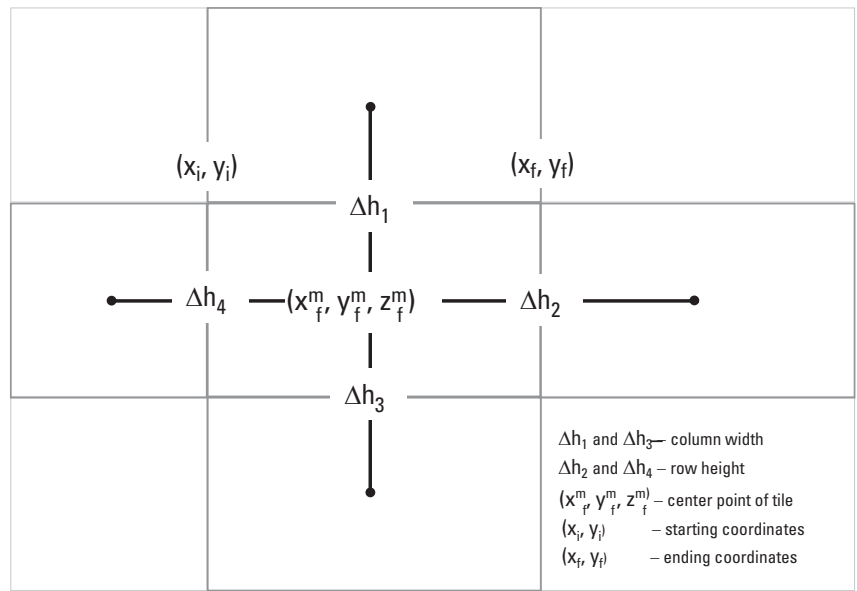

Figure 42. Grid and tile geometry for smoothing matrix $\mathbf{D}$.

\section{Computing the Location of the Center Point of Each Tile}

where

$$
\begin{gathered}
y_{f}^{m}=y_{m}-\left(z_{b}^{m}-z_{t}\right) \cot \delta \sin \phi \\
x_{f}^{m}=x_{m}+\left(z_{b}^{m}-z_{t}\right) \cot \delta \cos \phi, \text { and } \\
z_{b}^{m}=z_{t}+0.5\left(z_{b}-z_{t}\right) \\
x_{m}=x_{i}+0.5\left(x_{f}-x_{i}\right) \\
y_{m}=y_{f}+\left(y_{f}-y_{i}\right) \frac{x_{m}-x_{f}}{x_{f}-x_{i}},
\end{gathered}
$$

$\left(x_{i}, y_{i}\right)$ and $\left(x_{f}, y_{f}\right)$ are the (top) starting and ending coordinates of the tile (see also fig. 43), $\delta$ is the dip angle, and $\phi$ is the strike

$$
\phi=\tan ^{-1}\left(\frac{x_{f}-x_{i}}{y_{f}-y_{i}}\right) .
$$


A. Grid

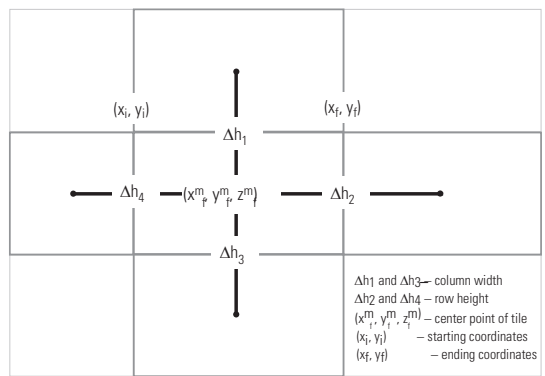

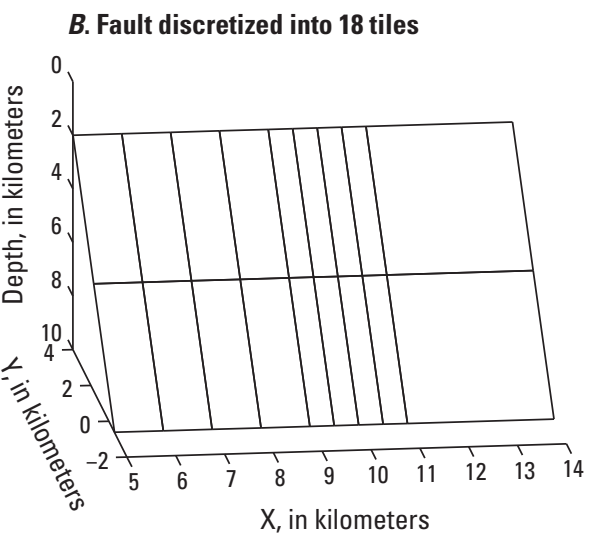

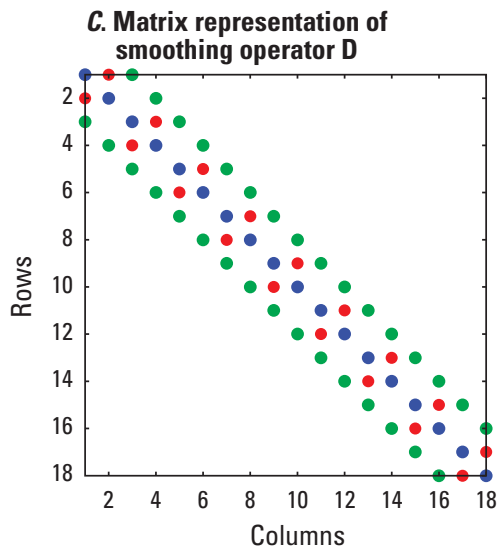

Figure 43. $A$, Grid used to compute the second-order finite-difference operator $\mathbf{D} ; B$, fault divided into $P=2$ rows, $N=9$ columns, and 18 tiles of different width; $C$, smoothing operator $\mathbf{D}$ for the fault in $B$. $\mathbf{D}$ has $(N P)^{2}=324$ elements, but the plot shows only the nonzero elements of the matrix. Blue, central diagonal; red, first diagonal above and below the central diagonal; green, Pth diagonal above and below the central diagonal.

\section{Computing Distances Between the Center Points of Tiles}

1. The vertical distance is

$$
\Delta h_{2}=\sqrt{\left(x_{f}^{m}(2)-x_{f}^{m}(1)\right)^{2}+\left(y_{f}^{m}(2)-y_{f}^{m}(1)\right)^{2}+\left(z_{b}^{m}(2)-z_{b}^{m}(1)\right)^{2}},
$$

where (1) and (2) indicate the component of the vectors;

2. The horizontal distance is

a. at the left edge (first column, where $n=1$ ),

$$
\Delta h_{1}(1: P)=\sqrt{\left(x_{f}^{m}(1+P)-x_{f}^{m}(1)\right)^{2}+\left(y_{f}^{m}(1+P)-y_{f}^{m}(1)\right)^{2}+\left(z_{b}^{m}(1+P)-z_{b}^{m}(1)\right)^{2}}
$$

b. at the right edge (last column, where $n=N$ ),

$$
\begin{aligned}
& \Delta h_{3}(N P+1-P: N P)= \\
& =\sqrt{\left(x_{f}^{m}(N P-P)-x_{f}^{m}(N P)\right)^{2}+\left(y_{f}^{m}(N P-P)-y_{f}^{m}(N P)\right)^{2}+\left(z_{b}^{m}(N P-P)-z_{b}^{m}(N P)\right)^{2}}
\end{aligned}
$$

where $P$ is the number of rows of tiles, $N$ is the number of columns of tiles, and $\boldsymbol{D}$ is a square matrix with the number of rows number of columns $P N$; and

c. from the central columns (where $2 \leq n \leq N-1$ ),

$$
\begin{aligned}
& \Delta h_{1}(1+(n-1) P: n P)= \\
& \quad=\sqrt{\left(x_{f}^{m}(n P-P)-x_{f}^{m}(n P)\right)^{2}+\left(y_{f}^{m}(n P-P)-y_{f}^{m}(n P)\right)^{2}+\left(z_{b}^{m}(n P-P)-z_{b}^{m}(n P)\right)^{2}} \text { and } \\
& \Delta h_{3}(1+(n-1) P: n P)= \\
& \quad=\sqrt{\left(x_{f}^{m}(n P+P)-x_{f}^{m}(n P)\right)^{2}+\left(y_{f}^{m}(n P+P)-y_{f}^{m}(n P)\right)^{2}+\left(z_{b}^{m}(n P+P)-z_{b}^{m}(n P)\right)^{2}} .
\end{aligned}
$$




\section{Computing the Smoothing Operator D}

The smoothing operator $\mathbf{D}$ is a second-order finite difference operator (equation 143). As the slip is determined on discrete tiles (figs. 42 and 43), $\nabla^{2} \mathbf{s}=\mathbf{D s}$ is a two-dimensional, second-order finite-difference sum

$$
\frac{s_{i-1, j}-2 s_{i, j}+s_{i+1, j}}{\left(\Delta h_{1}+\Delta h_{3}\right)^{2}}+\frac{s_{i, j-1}-2 s_{i, j}+s_{i, j+1}}{4 \Delta h_{2}^{2}} .
$$

The matrix implementing $\mathbf{D}$ is always zero, except along a limited number of diagonals (fig. 43C): the central diagonal and the first and Pth diagonals above and below the central diagonal, where $P$ is the number of rows of tiles on the fault (fig. $43 B$ ).

\section{Case 1-Fault does not Break the Surface}

1. First, compute the central diagonal (0)

$$
d=-2\left(\frac{1}{\Delta h_{1} \Delta h_{3}}+\frac{1}{\Delta h_{2}^{2}}\right)
$$

2. diagonal $(+1)$

$$
d p_{1}=\frac{1}{\Delta h_{2}^{2}}, \quad d p_{1}((1: N) P)=0, \quad d p_{1}=d p_{1}(1: N P-1)
$$

3. diagonal $(-1)$

$$
d m_{1}=\frac{1}{\Delta h_{2}^{2}}, \quad d m_{1}((1: N) P+1)=0 \quad d m_{1}=d m_{1}(2: N P),
$$

4. diagonal $(+\mathrm{P})$

$$
\overline{\mathrm{dp}_{4}}=\frac{2}{\Delta h_{3}\left(\Delta \mathrm{h}_{1}+\Delta h_{3}\right)}, \quad \mathrm{dp}_{4}=\overline{\mathrm{dp}_{4}}(1: \mathrm{NP}-\mathrm{P}) \text {, and finally }
$$

5. diagonal (-P)

$$
\overline{d m_{4}}=\frac{2}{\Delta h_{1}\left(\Delta h_{1}+\Delta h_{3}\right)}, \quad d m_{4}=\overline{d m_{4}}(1+P: N P)
$$




\section{Case 2-Fault Breaks the Surface}

1. First, compute the central diagonal (0)

$$
d(1)=\frac{1}{\Delta h_{2}(1)}, \quad d((1: N-1) P+1)=\frac{1}{\Delta h_{2}((1: N-1) P+1)},
$$

2. diagonal $(+1)$

$$
d p_{1}(1)=-\frac{1}{\Delta h_{2}(1)}, \quad d p_{1}((1: N-1) P+1)=-\frac{1}{\Delta h_{2}((1: N-1) P+1)}
$$

3. diagonal (-1)

$$
d m_{1}((1: N-1) P)=0
$$

4. diagonal $(+\mathrm{P})$ - this condition allows slip in the upper row of tiles to be nonzero when the fault breaks the surface (that is, produces measurable surface offsets)

$$
d p_{4}(1)=0, \quad d p_{4}((1: N-2) P+1)=0, \text { and finally }
$$

5. diagonal (-P) - see note for step 4 .

$$
d m_{4}(1)=0, \quad d m_{4}((1: N-2) P+1)=0
$$

\section{Case 3-Dike Opening at the Base of the Dislocation}

1. First, compute the central diagonal (0)

$$
d((1: N) P)=\frac{1}{\Delta h_{2}((1: N) P)}
$$

2. diagonal $(+1)$

$$
d p_{1}((1: N-1) P)=0
$$

3. diagonal (-1)

$$
\begin{gathered}
d m_{1}(P-1)=-\frac{1}{\Delta h_{2}(P-1)} \\
d m_{1}((1: N-1) P+P-1)=-\frac{1}{\Delta h_{2}((1: N-1) P+P-1)}
\end{gathered}
$$


4. diagonal $(+\mathrm{P})$ - this condition allows slip in the lower row of tiles to be nonzero when the dike opens at the bottom

$$
d p_{4}((1: N-1) P)=0 \text {, and finally }
$$

5. compute diagonal (-P) — see note for step 4.

$$
d m_{4}((1: N-1) P)=0
$$

\section{Matrix}

$$
\mathbf{D}=\operatorname{diag}(d)+\operatorname{diag}\left(d p_{1}, 1\right)+\operatorname{diag}\left(\operatorname{dm}_{1},-1\right)+\operatorname{diag}\left(d p_{4}, P\right)+\operatorname{diag}\left(d_{4},-P\right)
$$

where $\operatorname{diag}(v, k)$ is a function that returns a square matrix of order $n+|k|$ with the elements of $v$ on the $k t h$ diagonal when $v$ is a vector of $n$ components; $k=0$ represents the main diagonal, $k>0$ a diagonal above the main diagonal, and $k<0$ a diagonal below the main diagonal.

\section{Verification}

The algorithm for the smoothing operator $\mathbf{D}$ was tested by using the fault geometry shown in figure 44 and table 36. Following equations 143 and 151, we applied $\mathbf{D}$ to the vector of fault slips and compared the results to $\nabla^{2} \mathbf{s}$ (fig. 45), which is computed numerically by using finite differences (appendix 1).

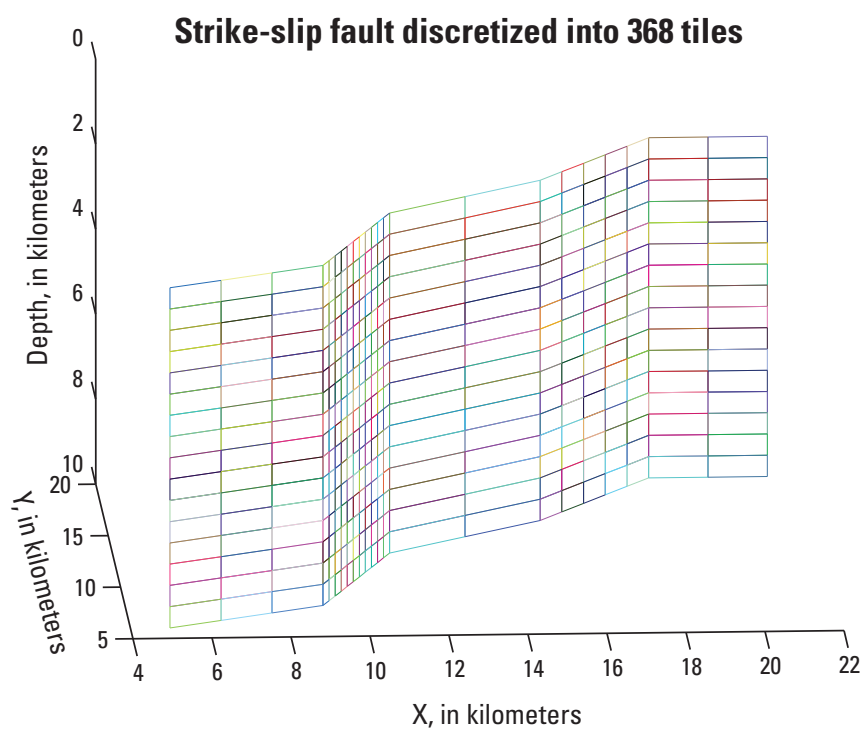

Figure 44. Fault geometry used to validate the algorithm for the smoothing operator D. See table 36 for parameters. 
Table 36. Values of fault parameters.

[Fault geometry is for the right-lateral strike-slip fault shown in figure 44. Segments were divided into tiles (or patches) by using $d L=\left[\begin{array}{lllll}2.0 & 0.5 & 3.0 & 1.0 & 1.5\end{array}\right]$ kilometers and $d W=0.5$ kilometers. $x_{\mathrm{i}}, y_{i}, x_{f}$, and $y_{f}$, coordinates of the initial and final positions along the top edges of the fault segments; $U_{1}$, final slip of segment; $z_{t}$ and $z_{b}$, coordinates of the top and bottom of the segment]

\begin{tabular}{ccccccccc}
\hline Segment & $\begin{array}{c}\boldsymbol{x}_{\boldsymbol{i}} \\
\text { (kilometers) }\end{array}$ & $\begin{array}{c}\boldsymbol{y}_{\boldsymbol{i}} \\
\text { (kilometers) }\end{array}$ & $\begin{array}{c}\boldsymbol{x}_{\boldsymbol{f}} \\
\text { (kilometers) }\end{array}$ & $\begin{array}{c}\boldsymbol{y}_{\boldsymbol{f}} \\
\text { (kilometers) }\end{array}$ & $\begin{array}{c}\boldsymbol{U}_{\mathbf{1}} \\
\text { (meters) }\end{array}$ & $\begin{array}{c}\text { Dip angle } \\
\text { (degrees) }\end{array}$ & $\begin{array}{c}\mathbf{z}_{\mathbf{t}} \\
\text { (kilometers) }\end{array}$ & $\begin{array}{c}\mathbf{z}_{\mathbf{b}} \\
\text { (kilometers) }\end{array}$ \\
\hline 1 & 5 & 6 & 9 & 8 & 1 & 10 & 2 \\
2 & 9 & 8 & 11 & 13 & 0.5 & 90 & 2 \\
3 & 11 & 13 & 15 & 16 & 1.5 & 90 & 2 \\
4 & 15 & 16 & 18 & 20 & 0.5 & 90 & 10 & 2 \\
5 & 18 & 20 & 21 & 20 & 0.75 & 90 & 10 \\
\hline
\end{tabular}
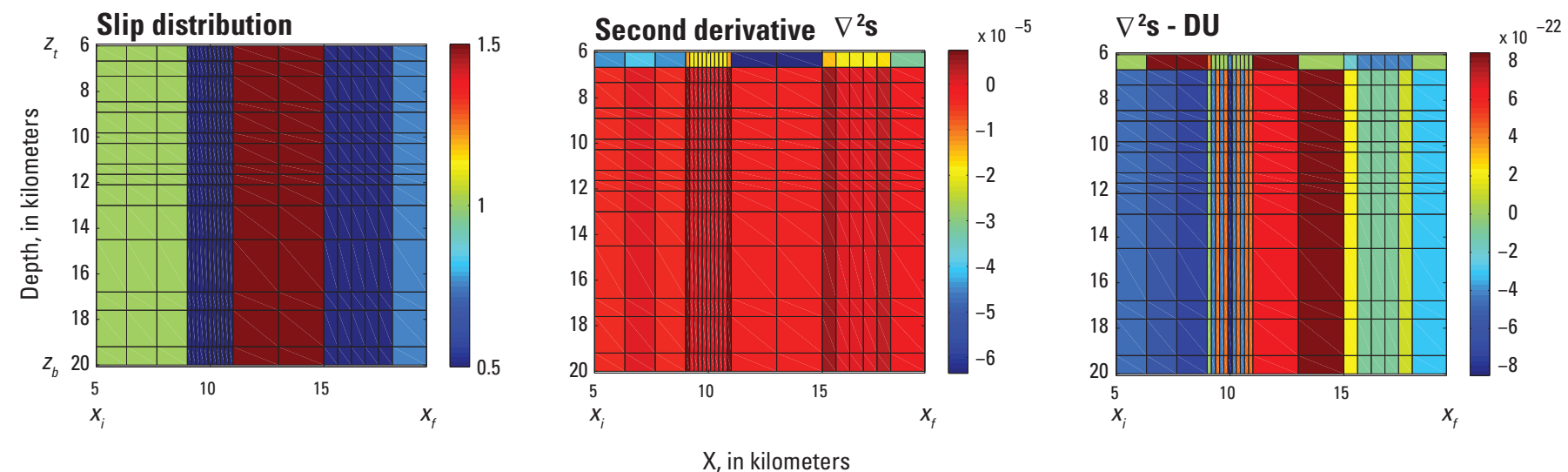

Figure 45. Color maps for the smoothing operator, slip distribution, and finite difference second derivative $\nabla^{2} \mathbf{s}$ (appendix 1) for the fault geometry shown in figure 44 . The smoothing operator $\mathbf{D}$ is a diagonal matrix with a number of elements equal to the square of the number of tiles (fig. 44). The difference between the finite difference second derivative $\nabla^{2} \mathbf{s}$ and DU is approximately 10-22. $x_{i}$ and $x_{f}$, horizontal east coordinate for the left and right corner of the fault; $z_{t}$, depth of the top edge of the fault; $z_{b}$, depth of the bottom edge of the fault. 


\section{Example for a Right-Lateral Strike-Slip Fault}

The example is based on the fault geometry shown below (fig. 46 and table 37). Numerical values of the smoothing operator D, slip vector U, product DU, and finite-difference second derivative $(\mathrm{grad})^{2} \mathrm{~S}$ are in tables 38-41.

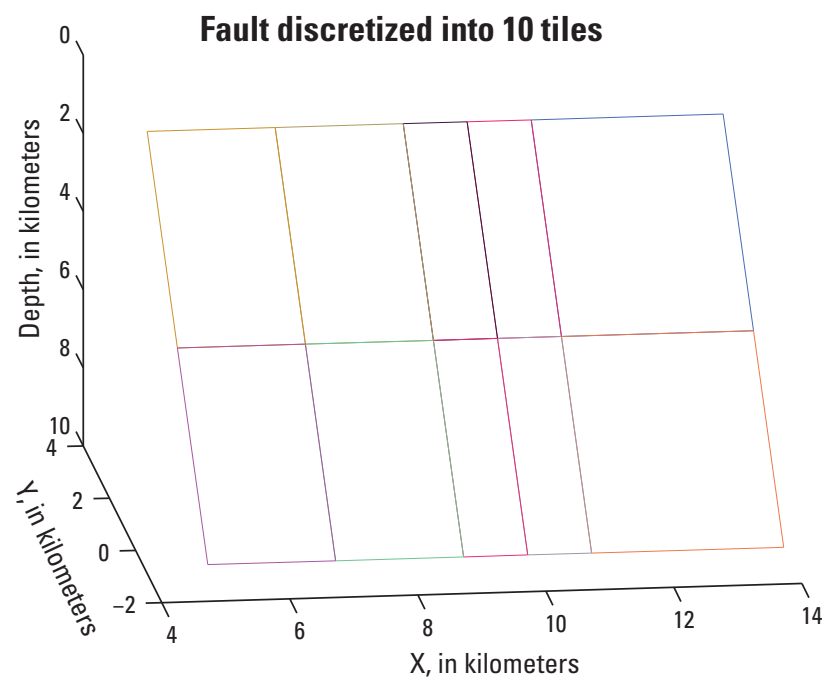

Figure 46. Fault geometry employed in the example. See table 37 for fault parameters.

Table 37. Values of fault parameters.

[Fault geometry is for the right-lateral strike-slip fault shown in figure 46. Segments were divided into tiles by using $d L=\left[\begin{array}{lll}3.0 & 1.0 & 3.0\end{array}\right]$ kilometers and $d W=5.0$ kilometers. $x_{\mathrm{i}}, y_{\mathrm{i}}, x_{\mathrm{f}}$, and $y_{f}$, coordinates of the initial and final positions along the top edges of the fault segments; $U_{3}$, final opening dislocation of segment; $\mathrm{z}_{\mathrm{t}}$ and $\mathrm{z}_{\mathrm{b}}$, coordinates of the top and bottom of the segment]

\begin{tabular}{ccccccccc}
\hline Segment $^{\mathrm{a}}$ & $\begin{array}{c}\boldsymbol{x}_{\boldsymbol{i}} \\
\text { (kilometers) }\end{array}$ & $\begin{array}{c}\boldsymbol{y}_{\boldsymbol{i}} \\
\text { (kilometers) }\end{array}$ & $\begin{array}{c}\boldsymbol{x}_{\boldsymbol{f}} \\
\text { (kilometers) }\end{array}$ & $\begin{array}{c}\boldsymbol{y}_{\boldsymbol{f}} \\
\text { (kilometers) }\end{array}$ & $\begin{array}{c}\boldsymbol{U}_{\mathbf{1}} \\
\text { (meters) }\end{array}$ & $\begin{array}{c}\text { Dip angle } \\
\text { (degrees) }\end{array}$ & $\begin{array}{c}\boldsymbol{z}_{\boldsymbol{t}} \\
\text { (kilometers) }\end{array}$ & $\begin{array}{c}\boldsymbol{z}_{\boldsymbol{b}} \\
\text { (kilometers) }\end{array}$ \\
\hline 1 & 5 & 4 & 9 & 4 & 1 & 60 & 2 & 10 \\
2 & 9 & 4 & 11 & 4 & 0.5 & 60 & 2 \\
3 & 11 & 4 & 14 & 4 & 1.5 & 60 & 2 \\
\hline
\end{tabular}


Table 38. Values of the smoothing operator D.

[The reader should multiply the elements below by $10^{-5}$. Value of $\mathrm{D}$ is in (kilometers) ${ }^{-2}$ ]

\begin{tabular}{|c|c|c|c|c|c|c|c|c|c|c|}
\hline & 1 & 2 & 3 & 4 & 5 & 6 & 7 & 8 & 9 & 10 \\
\hline 1 & -0.0594 & 0.0047 & 0.0250 & 0 & 0 & 0 & 0 & 0 & 0 & 0 \\
\hline 2 & 0.0047 & -0.0594 & 0 & 0.0250 & 0 & 0 & 0 & 0 & 0 & 0 \\
\hline 3 & 0.0286 & 0 & -0.0760 & 0.0047 & 0.0381 & 0 & 0 & 0 & 0 & 0 \\
\hline 4 & 0 & 0.0286 & 0.0047 & -0.0760 & 0 & 0.0381 & 0 & 0 & 0 & 0 \\
\hline 5 & 0 & 0 & 0.0533 & 0 & -0.1427 & 0.0047 & 0.0800 & 0 & 0 & 0 \\
\hline 6 & 0 & 0 & 0 & 0.0533 & 0.0047 & -0.1427 & 0 & 0.0800 & 0 & 0 \\
\hline 7 & 0 & 0 & 0 & 0 & 0.0667 & 0 & -0.1094 & 0.0047 & 0.0333 & 0 \\
\hline 8 & 0 & 0 & 0 & 0 & 0 & 0.0667 & 0.0047 & -0.1094 & 0 & 0.0333 \\
\hline 9 & 0 & 0 & 0 & 0 & 0 & 0 & 0.0250 & 0 & -0.0594 & 0.0047 \\
\hline 10 & 0 & 0 & 0 & 0 & 0 & 0 & 0 & 0.0250 & 0.0047 & -0.0594 \\
\hline
\end{tabular}

Table 39. Values of the slip vector $\mathrm{U}$.

[Values of $\mathbf{U}$ are in meters]

\begin{tabular}{|c|c|c|c|c|c|c|c|c|c|c|}
\hline & 1 & 2 & 3 & 4 & 5 & 6 & 7 & 8 & 9 & 10 \\
\hline 1 & 1.0 & 1.0 & 1.0 & 1.0 & 0.5 & 0.5 & 0.5 & 0.5 & 1.5 & 1.5 \\
\hline
\end{tabular}

Table 40. Values of the product DU.

[Values of DU are in meters per square kilometer].

\begin{tabular}{|ccccccc}
\hline & $\mathbf{1}$ & $\mathbf{2}$ & $\mathbf{3}$ & $\mathbf{4}$ & $\mathbf{5}$ \\
\cline { 3 - 7 } $\mathbf{1}$ & -0.2969 & -0.2374 & 0.2432 & 0.3099 & -0.6953 \\
$\mathbf{2}$ & -0.2969 & -0.2374 & 0.2432 & 0.3099 & -0.6953
\end{tabular}

Table 41. Values of the finite-difference second derivative $\nabla^{2} \mathbf{s}$.

[Values $\nabla^{2} \mathbf{S}$ of are in meters per square kilometer. See Appendix 1.]

\begin{tabular}{|cccccc}
\hline \multicolumn{1}{r}{} & $\mathbf{1}$ & $\mathbf{2}$ & $\mathbf{3}$ & $\mathbf{4}$ & $\mathbf{5}$ \\
$\mathbf{n n y y y y}$ & -0.2969 & -0.2374 & 0.2432 & 0.3099 & -0.6953 \\
$\mathbf{2}$ & -0.2969 & -0.2374 & 0.2432 & 0.3099 & -0.6953
\end{tabular}




\section{Summary}

This report is a practical reference for readers interested in applying mathematical models to investigate volcano and earthquake physics. The report emphasizes the application of analytical models to fit and interpret ground deformation. Although analytical models are based on numerous simplifications (for example, the assumption that the crust is a homogenous, isotropic, elastic, flat half-space) that make the set of differential equations describing the problem tractable, they can take into account a vast array of source geometries. The careful use of analytical models, together with high-quality data sets, can yield valuable insights into the nature of the deformation source.

The paper introduces analytical formulation that describe the deformation caused by magma chambers, magma conduit, sill, dikes and faults, and provides tables with numerical examples and Matlab scripts to aid in the use and coding of the formulas. All formulas have been checked for errors and verified against numerical models (COULOMB 3.3; http:// earthquake.usgs.gov/research/modeling/coulomb/; FEM models). A zipped file with MATLAB scripts can be downloaded from http://pubs.usgs.gov/tm/13/b1.

Suggestions about new models that could be included in future revisions, reports of existing bugs in the Matlab scripts (or errors in the formulas) and comments on how to improve this publication will be greatly appreciated.

\section{References Cited}

Amoruso, Antonella, and Crescentini, Luca, 2009, Shape and volume change of pressurized ellipsoidal cavities from deformation and seismic data: Journal of Geophysical Research, v. 114, B02210 (also available at http://dx.doi.org/ 10.1029/2008JB005946).

Barnhart, W.D., and Lohman, R.B., 2010, Automated fault model discretization for inversions for coseismic slip distributions: Journal of Geophysical Research, v. 115, B10419, 17 p. (also available at http://dx.doi.org/ 10.1029/2010JB007545).

Cervelli, P.F., Fournier, T.J., Freymueller, J.T., and Power, J.A., 2006, Ground deformation associated with the precursory unrest and early phases of the January 2006 eruption of Augustine Volcano, Alaska: Geophysical Research Letters, v. 33, L18304 (also available at http://dx.doi.org/ 10.1029/2006GL027219).

Dawson, John, and Woods, Alex, 2010, ITRF to GDA94 Coordinate Transformations: Journal of Applied Geodesy, v. 4, 189-199 (also available at http://dx.doi.org/ 10.1515/ JAG.2010.019)

Defense Mapping Agency (DMA), 1989, The Universal Grids-Universal Transverse Mercator (UTM) and Universal Polar Stereographic (UPS): Fairfax, Va., DMA Technical Manual DMATM 8358.2.

Dzurisin, Daniel, 2003, A comprehensive approach to monitoring volcano deformation as a window on the eruption cycle: Review of Geophysics, v. 41, no. 1, 1001 p. (also available at http://dx.doi.org/10.1029/2001RG000107).

Fialko, Y., Khazan, Y., and Simons, M., 2001, Deformation due to a pressurized horizontal circular crack in an elastic half-space, with applications to volcano geodesy: Geophysical Journal International, v. 146, no.1, p. 181-190.

Hoffmann-Wellenhof, B., Lichtenegger, H., and Collins, J., 1997, Global positioning systemTheory and practice (4th revised ed.): New York and Vienna, Springer, 389 p. 
Jónsson, S., Zebker, H., Segall, P., and Amelung, F., 2002, Fault slip distribution of the 1999 M7.2 Hector Mine earthquake, California, estimated from satellite radar and GPS measurements: Bulletin of the Seismological Society of America, v. 92, no. 4, p. 1377-1389.

Masterlark, Timothy, 2007, Magma instrusion and deformation predictions-Sensitivities to the Mogi assumptions: Journal of Geophysical Research, v. 112, no. B06419, 17 p. (also available at http://dx.doi.org/10.1029/2006JB004860).

McTigue, D.F., 1987, Elastic stress and deformation near a finite spherical magma bodyResolution of the point source paradox: Journal of Geophysical Research, v. 92, no. B12, p. 12931-12940 (also available at http://dx.doi.org/10.1029/JB092iB12p12931).

National Imagery and Mapping Agency (NIMA), 2000, Department of Defense World Geodetic System 1984-Its definition and relationship with local geodetic systems: Technical Report TR8350.2.

National Imagery and Mapping Agency (NIMA), 2004, Implementation of the World Geodetic System 1984 (WGS 84) Reference Frame G1150. Addendum to NIMA TR 8350.2.

Newman, A.V., Dixon, T.H., and Gourmelen, N., 2006, A four-dimensional viscoelastic deformation model for Long Valley Caldera, California, between 1995 and 2000: Journal of Volcanology and Geothermal Research, v. 150, p. 244-269.

Okada, Yoshimitsu, 1985, Surface deformation due to shear and tensile faults in a half-space: Bulletin of the Seismological Society of America, v. 75, no. 4, p. 1135-1154.

Okada, Yoshimitsu, 1992, Internal deformation due to shear and tensile faults in a half-space: Bulletin of the Seismological Society of America, v. 82, no. 2, p. 1018-1040.

Press, W.H., Teukolsky, S.A., Vetterling, W.T., and Flannery, B.P., 1992, Numerical recipes in Fortran 77-The Art of Scientific Computing (2d ed.), v. 1: Cambridge, Cambridge University Press, $921 \mathrm{p}$.

Segall, Paul, 2010, Earthquake and volcano deformation: Princeton, N.J., Princeton University Press, $458 \mathrm{p}$.

Tiampo, K.F., Rundle, J.B., Fernandez, J., and Langbein, J.O., 2000, Spherical and ellipsoidal volcanic sources at Long Valley caldera, California, using a genetic algorithm inversion technique: Journal of Volcanology and Geothermal Research, v. 102, no. 3, p. 189-206.

U.S. Army, 1987, Standard UTM-to-GEO/GEO-to-UTM Coordinate Conversion Algorithm: Technical Manual TM 5-241-8 (accessed on August 2, 2012 at http://ntrs.nasa.gov/archive/ nasa/casi.ntrs.nasa.gov/19890002875_1989002875.pdf).

Yang, X.-M., Davis, P.M., and Dieterich, J.H., 1988, Deformation from inflation of a dipping finite prolate spheroid in an elastic half-space as a model for volcanic stressing: Journal of Geophysical Research, v. 93, no. B5, p. 4249-4257. 


\section{Appendix 1. Mathematical Methods for Computing Displacement}

\section{Displacement Gradient Tensor}

The partial differentiation of the displacement vector $\mathbf{u}$ with respect to the spatial coordinates yields the displacement gradient tensor $\nabla \mathbf{u}$. The finite strains measured by a Gladwin Tensor Strainmeter (GTSM) can be expressed as a function of the displacementgradient tensor. We can compute the components of the displacement gradient tensor using the finite-difference derivatives (Press and others, 1992, p. 180):

$$
\begin{aligned}
\frac{\partial u_{x}}{\partial x} & =\frac{u_{x}(x+h, y, z)-u_{x}(x-h, y, z)}{2 h}+o\left(h^{2}\right) \\
\frac{\partial u_{x}}{\partial y} & =\frac{u_{x}(x, y+h, z)-u_{x}(x, y-h, z)}{2 h}+o\left(h^{2}\right) \\
\frac{\partial u_{y}}{\partial x} & =\frac{u_{y}(x+h, y, z)-u_{y}(x-h, y, z)}{2 h}+o\left(h^{2}\right) \\
\frac{\partial u_{y}}{\partial y} & =\frac{u_{y}(x, y+h, z)-u_{y}(x, y-h, z)}{2 h}+o\left(h^{2}\right)
\end{aligned}
$$

The horizontal derivatives of the vertical deformation correspond to the east and north components of tilt:

$$
\begin{gathered}
\tau_{E}=\frac{\partial u_{z}}{\partial x}=\frac{u_{z}(x+h, y, z)-u_{z}(x-h, y, z)}{2 h}+o\left(h^{2}\right) . \\
\tau_{N}=\frac{\partial u_{z}}{\partial y}=\frac{u_{z}(x, y+h, z)-u_{z}(x, y-h, z)}{2 h}+o\left(h^{2}\right)
\end{gathered}
$$

\section{Length Change to an Electronic-Distance-Meter Base Line}

The change in length of an electronic-distance-meter (EDM) baseline $\left(\delta r_{a b}\right)$ is given by the sum of the projections of the radial deformations at the two benchmarks along the baseline (fig. 1-1)

$$
\delta r_{a b}=u_{r a} \cos \theta_{a}+u_{r b} \cos \theta_{b},
$$

where

$$
\cos \theta_{a}=\frac{r_{a}^{2}+r_{a b}^{2}-r_{b}^{2}}{2 r_{a} r_{a b}} \text { and } \cos \theta_{b}=\frac{r_{b}^{2}+r_{a b}^{2}-r_{a}^{2}}{2 r_{b} r_{a b}}
$$

It is also possible to write equation 1-3 in the vector notation

$$
\delta r_{a b}=\overrightarrow{u_{r a}} \cdot \frac{\overrightarrow{r_{a b}}}{\left\|r_{a b}\right\|}+\overrightarrow{u_{r b}} \cdot \frac{\overrightarrow{r_{a b}}}{\left\|r_{a b}\right\|} .
$$




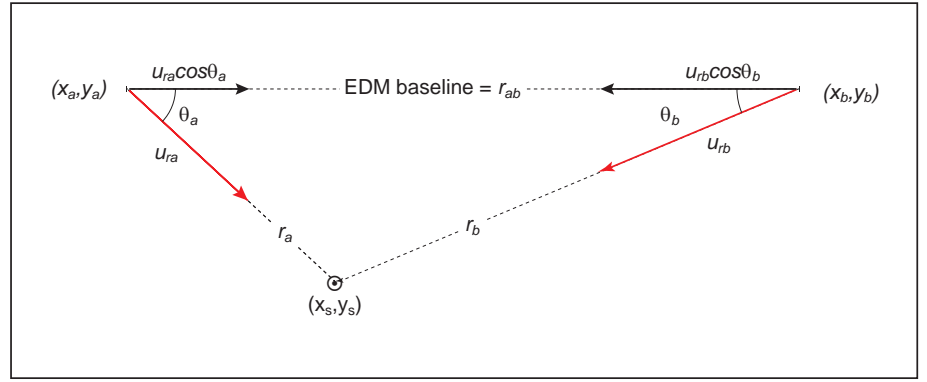

Figure 1-1. Geometry of a length change to an electronicdistance-meter (EDM) baseline. $\left(x_{a}, y_{a}\right)$, Cartesian coordinates of the EDM base station; $\left(x_{b}, y_{b}\right)$, Cartesian coordinates of the EDM target station; $\left(x_{s}, y_{s}\right)$, Cartesian coordinates of source location; $r_{a b}$, EDM baseline length; $r_{a}$, Cartesian distance between EDM base and source; $r_{b}$, Cartesian distance between EDM target and source; $\theta_{a}$ and $\theta_{b^{\prime}}$ angles; $u_{r a}$ and $u_{r b}$, radial deformation.

\section{Second Derivative of the Slip Vector}

We use this formulation for second derivative of the slip vector s (after Zwillinger, 1997 , p. 661) to test the smoothing operator $\mathbf{D}$. The second derivative of $\mathbf{s}$ is defined as

$$
\nabla^{2} \mathbf{s}=\left(\frac{\partial^{2}}{\partial x^{2}}+\frac{\partial^{2}}{\partial y^{2}}+\frac{\partial^{2}}{\partial z^{2}}\right) \mathbf{s}
$$

where

$\mathrm{S} \quad$ is a matrix describing the slip distribution along the fault.

The irregular grid and index convention used are shown in figure 1-2 below.

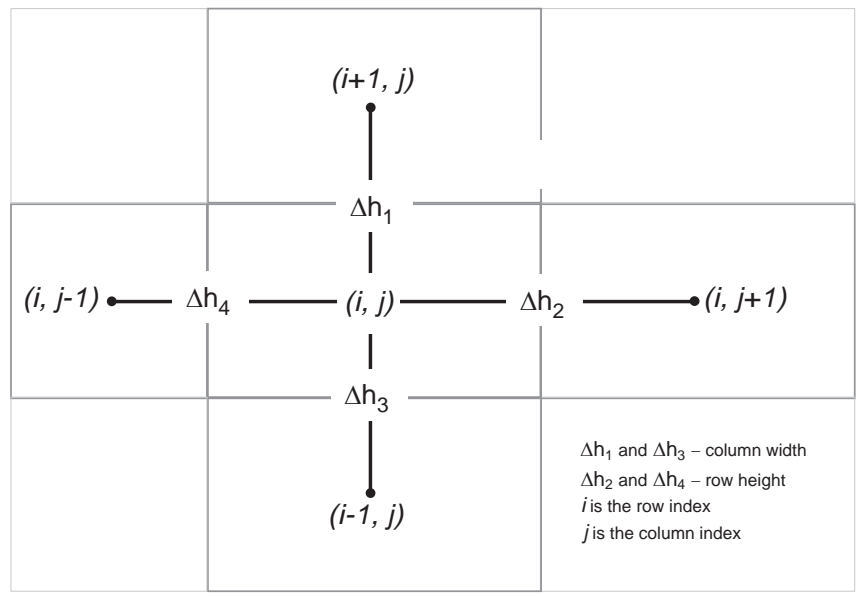

Figure 1-2. Irregular grid geometry, after Zwillinger (1997, p. 661). 
The Laplacian is computed in three steps:

Step 1. Compute the Location of the Center Point of Each Tile

where

$$
\begin{array}{ll}
x_{f}^{m}=x_{m}+\left(z_{b}^{m}-z_{t}\right) \cot \delta \cos \phi, & \text { East } \\
y_{f}^{m}=y_{m}-\left(z_{b}^{m}-z_{t}\right) \cot \delta \sin \phi, & \text { North } \\
z_{b}^{m}=z_{t}+0.5\left(z_{b}-z_{t}\right), & \text { Up }
\end{array}
$$

Step 2. Build Matrices with East, North, and Up Components of the Center and the Slip of the Tile

$$
\begin{aligned}
J & =J+P & & \text { index } \\
K & =K-P & & \text { index } \\
M X(1+J: K, n) & =x_{f}^{m}(1+J: K) & & \text { East } \\
M Y(1+J: K, n) & =y_{f}^{m}(1+J: K) & & \text { North } \\
M Z(1+J: K, n) & =z_{b}^{m}(1+J: K) & & \text { Up } \\
M U(1+J: K, n) & =U(1+J: K) & & \text { Slip }
\end{aligned}
$$

where $n=1, \ldots, N$.

Step 3. Compute the Laplacian L

Internal Tiles $(i=2, \ldots, P-1$ and $j=2, \ldots, N-1)$,

$$
\begin{aligned}
\Delta h_{4} & =\sqrt{\left(M X_{i, j}-M X_{i-1, j}\right)^{2}+\left(M Y_{i, j}-M Y_{i-1, j}\right)^{2}+\left(M Z_{i, j}-M Z_{i-1, j}\right)^{2}} \\
\Delta h_{3} & =\sqrt{\left(M X_{i, j+1}-M X_{i, j}\right)^{2}+\left(M Y_{i, j+1}-M Y_{i, j}\right)^{2}+\left(M Z_{i, j+1}-M Z_{i, j}\right)^{2}} \\
\Delta h_{2} & =\sqrt{\left(M X_{i+1, j}-M X_{i, j}\right)^{2}+\left(M Y_{i+1, j}-M Y_{i, j}\right)^{2}+\left(M Z_{i+1, j}-M Z_{i, j}\right)^{2}} \\
\Delta h & =\sqrt{\left(M X_{i, j}-M X_{i, j-1}\right)^{2}+\left(M Y_{i, j}-M Y_{i, j-1}\right)^{2}+\left(M Z_{i, j}-M Z_{i, j-1}\right)^{2}}
\end{aligned}
$$

and

$$
\begin{array}{r}
L_{i, j}=2\left[\frac{M U_{i, j-1}}{\Delta h_{1}\left(\Delta h_{1}+\Delta h_{3}\right)}+\frac{M U_{i+1, j}}{\Delta h_{2}\left(\Delta h_{2}+\Delta h_{4}\right)}+\frac{M U_{i, j+1}}{\Delta h_{3}\left(\Delta h_{1}+\Delta h_{3}\right)}+\cdots\right. \\
\left.\quad \cdots+\frac{M U_{i-1, j}}{\Delta h_{4}\left(\Delta h_{2}+\Delta h_{4}\right)}-M U_{i, j}\left(\frac{1}{\Delta h_{1} \cdot \Delta h_{3}}+\frac{1}{\Delta h_{2} \cdot \Delta h_{4}}\right)\right]
\end{array}
$$


Top edge ( $i=1$ ) where the Fault does not Break the Earth's Surface

Left tile $(j=1)$

$$
\begin{aligned}
\Delta h_{2} & =\sqrt{\left(M X_{i+1, j}-M X_{i, j}\right)^{2}+\left(M Y_{i+1, j}-M Y_{i, j}\right)^{2}+\left(M Z_{i+1, j}-M Z_{i, j}\right)^{2}} \\
\Delta h_{4} & =\Delta h_{2} \\
\Delta h_{3} & =\sqrt{\left(M X_{i, j+1}-M X_{i, j}\right)^{2}+\left(M Y_{i, j+1}-M Y_{i, j}\right)^{2}+\left(M Z_{i, j+1}-M Z_{i, j}\right)^{2}} \\
\Delta h_{1} & =\Delta h_{3}
\end{aligned}
$$

and

$$
L_{i, j}=2\left[\frac{M U_{i+1, j}}{\Delta h_{2}\left(\Delta h_{2}+\Delta h_{4}\right)}+\frac{M U_{i, j+1}}{\Delta h_{3}\left(\Delta h_{1}+\Delta h_{3}\right)}-M U_{i, j}\left(\frac{1}{\Delta h_{1} \cdot \Delta h_{3}}+\frac{1}{\Delta h_{2} \cdot \Delta h_{2}}\right)\right] .
$$

Internal tiles $(j=2, \ldots, N-1)$

$$
\begin{aligned}
& \Delta h_{2}=\sqrt{\left(M X_{i+1, j}-M X_{i, j}\right)^{2}+\left(M Y_{i+1, j}-M Y_{i, j}\right)^{2}+\left(M Z_{i+1, j}-M Z_{i, j}\right)^{2}} \\
& \Delta h_{4}=\Delta h_{2} \\
& \Delta h_{3}=\sqrt{\left(M X_{i, j+1}-M X_{i, j}\right)^{2}+\left(M Y_{i, j+1}-M Y_{i, j}\right)^{2}+\left(M Z_{i, j}-M Z_{i, j}\right)^{2}} \\
& \Delta h_{1}=\sqrt{\left(M X_{i, j}-M X_{i, j-1}\right)^{2}+\left(M Y_{i, j}-M Y_{i, j-1}\right)^{2}+\left(M Z_{i, j}-M Z_{i, j-1}\right)^{2}}
\end{aligned}
$$

and

$$
L_{i, j}=2\left[\frac{M U_{i, j-1}}{\Delta h_{1}\left(\Delta h_{1}+\Delta h_{3}\right)}+\frac{M U_{i+1, j}}{\Delta h_{2}\left(\Delta h_{2}+\Delta h_{4}\right)}+\frac{M U_{i, j+1}}{\Delta h_{3}\left(\Delta h_{1}+\Delta h_{3}\right)}-M U_{i, j}\left(\frac{1}{\Delta h_{1} \cdot \Delta h_{3}}+\frac{1}{\Delta h_{2} \cdot \Delta h_{2}}\right)\right] .
$$

Right tile $(j=N)$

$$
\begin{aligned}
\Delta h_{2} & =\sqrt{\left(M X_{i+1, j}-M X_{i, j}\right)^{2}+\left(M Y_{i+1, j}-M Y_{i, j}\right)^{2}+\left(M Z_{i+1, j}-M Z_{i, j}\right)^{2}} \\
\Delta h_{4} & =\Delta h_{2} \\
\Delta h_{1} & =\sqrt{\left(M X_{i, j}-M X_{i, j-1}\right)^{2}+\left(M Y_{i, j}-M Y_{i, j-1}\right)^{2}+\left(M Z_{i, j}-M Z_{i, j-1}\right)^{2}} \\
\Delta h_{3} & =\Delta h_{1}
\end{aligned}
$$

and

$$
L_{i, j}=2\left[\frac{M U_{i, j-1}}{\Delta h_{1}\left(\Delta h_{1}+\Delta h_{3}\right)}+\frac{M U_{i+1, j}}{\Delta h_{2}\left(\Delta h_{2}+\Delta h_{4}\right)}-M U_{i, j}\left(\frac{1}{\Delta h_{1} \cdot \Delta h_{3}}+\frac{1}{\Delta h_{2} \cdot \Delta h_{4}}\right)\right] .
$$


Top Edge $(i=1)$ where the Fault Breaks the Earth's Surface

If the fault breaks the surface, we must replace equations 1-12 to 1-17 with

$$
\begin{aligned}
\Delta h_{2} & =\sqrt{\left(M X_{i+1, j}-M X_{i, j}\right)^{2}+\left(M Y_{i+1, j}-M Y_{i, j}\right)^{2}+\left(M Z_{i+1, j}-M Z_{i, j}\right)^{2}} \\
L_{i, j} & =\frac{M U_{i+1, j}-M U_{i, j}}{\Delta h_{2}}, \quad j=1, \ldots, N
\end{aligned} .
$$

Bottom Edge $(i=P)$,Tensile Crack (Dike) is not Opening

Left tile $(j=1)$

$$
\begin{gathered}
\Delta h_{4}=\sqrt{\left(M X_{i, j}-M X_{i-1, j}\right)^{2}+\left(M Y_{i, j}-M Y_{i-1, j}\right)^{2}+\left(M Z_{i, j}-M Z_{i-1, j}\right)^{2}} \\
\Delta h_{3}=\sqrt{\left(M X_{i, j+1}-M X_{i, j}\right)^{2}+\left(M Y_{i, j+1}-M Y_{i, j}\right)^{2}+\left(M Z_{i, j+1}-M Z_{i, j}\right)^{2}}, \\
\Delta h_{2}=\Delta h_{4} \\
\Delta h_{1}=\Delta h_{3} \\
L_{i, j}=2\left[\frac{M U_{i, j+1}}{\Delta h_{3}\left(\Delta h_{1}+\Delta h_{3}\right)}+\frac{M U_{i-1, j}}{\Delta h_{4}\left(\Delta h_{2}+\Delta h_{4}\right)}-M U_{i, j}\left(\frac{1}{\Delta h_{1} \cdot \Delta h_{3}}+\frac{1}{\Delta h_{2} \cdot \Delta h_{4}}\right)\right] .
\end{gathered}
$$

Internal tiles $(j=2, \ldots, N-1)$

$$
\begin{gathered}
\Delta h_{4}=\sqrt{\left(M X_{i, j}-M X_{i-1, j}\right)^{2}+\left(M Y_{i, j}-M Y_{i-1, j}\right)^{2}+\left(M Z_{i, j}-M Z_{i-1, j}\right)^{2}} \\
\Delta h_{3}=\sqrt{\left(M X_{i, j+1}-M X_{i, j}\right)^{2}+\left(M Y_{i, j+1}-M Y_{i, j}\right)^{2}+\left(M Z_{i, j+1}-M Z_{i, j}\right)^{2}} \\
\Delta h_{2}=\Delta h_{4} \\
\Delta h_{1}=\sqrt{\left(M X_{i, j}-M X_{i, j-1}\right)^{2}+\left(M Y_{i, j}-M Y_{i, j-1}\right)^{2}+\left(M Z_{i, j}-M Z_{i, j-1}\right)^{2}} \\
L_{i, j}=2\left[\frac{M U_{i, j-1}}{\Delta h_{1}\left(\Delta h_{1}+\Delta h_{3}\right)}+\frac{M U_{i, j+1}}{\Delta h_{3}\left(\Delta h_{1}+\Delta h_{3}\right)}+\frac{M U_{i-1, j}}{\Delta h_{4}\left(\Delta h_{2}+\Delta h_{4}\right)}-M U_{i, j}\left(\frac{1}{\Delta h_{1} \cdot \Delta h_{3}}+\frac{1}{\Delta h_{2} \cdot \Delta h_{4}}\right)\right] .
\end{gathered}
$$


Right tile $(j=N)$

$$
\begin{aligned}
& \Delta h_{4}=\sqrt{\left(M X_{i, j}-M X_{i-1, j}\right)^{2}+\left(M Y_{i, j}-M Y_{i-1, j}\right)^{2}+\left(M Z_{i, j}-M Z_{i-1, j}\right)^{2}} \\
& \Delta h_{1}=\sqrt{\left(M X_{i, j}-M X_{i, j-1}\right)^{2}+\left(M Y_{i, j}-M Y_{i, j-1}\right)^{2}+\left(M Z_{i, j}-M Z_{i, j-1}\right)^{2}}, \\
& \Delta h_{3}=\Delta h_{1} \\
& \Delta h_{2}=\Delta h_{4} \\
& L_{i, j}=2\left[\frac{M U_{i, j-1}}{\Delta h_{1}\left(\Delta h_{1}+\Delta h_{3}\right)}+\frac{M U_{i-1, j}}{\Delta h_{4}\left(\Delta h_{2}+\Delta h_{4}\right)}-M U_{i, j}\left(\frac{1}{\Delta h_{1} \cdot \Delta h_{3}}+\frac{1}{\Delta h_{2} \cdot \Delta h_{4}}\right)\right] .
\end{aligned}
$$

\section{Bottom Edge $(i=P)$, the Tensile Crack (Dike) is Opening}

If the crack is opening, we must replace all the equations above with

$$
\begin{aligned}
& \Delta h_{4}=\sqrt{\left(M X_{i, j}-M X_{i-1, j}\right)^{2}+\left(M Y_{i, j}-M Y_{i-1, j}\right)^{2}+\left(M Z_{i, j}-M Z_{i-1, j}\right)^{2}} . \\
& L_{i, j}=\frac{M U_{i, j}-M U_{i-1, j}}{\Delta h_{4}}, \quad j=1, \ldots, N
\end{aligned}
$$

Left Edge $(i=2, \ldots, P-1$ and $j=1)$

$$
\begin{gathered}
\Delta h_{4}=\sqrt{\left(M X_{i, j}-M X_{i-1, j}\right)^{2}+\left(M Y_{i, j}-M Y_{i-1, j}\right)^{2}+\left(M Z_{i, j}-M Z_{i-1, j}\right)^{2}} \\
\Delta h_{3}=\sqrt{\left(M X_{i, j+1}-M X_{i, j}\right)^{2}+\left(M Y_{i, j+1}-M Y_{i, j}\right)^{2}+\left(M Z_{i, j+1}-M Z_{i, j}\right)^{2}}, \\
\Delta h_{2}=\sqrt{\left(M X_{i+1, j}-M X_{i, j}\right)^{2}+\left(M Y_{i+1, j}-M Y_{i, j}\right)^{2}+\left(M Z_{i+1, j}-M Z_{i, j}\right)^{2}} \\
\Delta h_{1}=\Delta h_{3} \\
L_{i, j}=2\left[\frac{M U_{i+1, j}}{\Delta h_{2}\left(\Delta h_{2}+\Delta h_{4}\right)}+\frac{M U_{i, j+1}}{\Delta h_{3}\left(\Delta h_{1}+\Delta h_{3}\right)}+\frac{M U_{i-1, j}}{\Delta h_{4}\left(\Delta h_{2}+\Delta h_{4}\right)}-M U_{i, j}\left(\frac{1}{\Delta h_{1} \cdot \Delta h_{3}}+\frac{1}{\Delta h_{2} \cdot \Delta h_{4}}\right)\right]
\end{gathered}
$$


Right Edge $(i=2, \ldots, P-1$ and $j=N)$

$$
\begin{gathered}
\Delta h_{1}=\sqrt{\left(M X_{i, j}-M X_{i, j-1}\right)^{2}+\left(M Y_{i, j}-M Y_{i, j-1}\right)^{2}+\left(M Z_{i, j}-M Z_{i, j-1}\right)^{2}} \\
\Delta h_{2}=\sqrt{\left(M X_{i+1, j}-M X_{i, j}\right)^{2}+\left(M Y_{i+1, j}-M Y_{i, j}\right)^{2}+\left(M Z_{i+1, j}-M Z_{i, j}\right)^{2}}, \\
\Delta h_{3}=\Delta h_{1} \\
\Delta h_{4}=\sqrt{\left(M X_{i, j}-M X_{i-1, j}\right)^{2}+\left(M Y_{i, j}-M Y_{i-1, j}\right)^{2}+\left(M Z_{i, j}-M Z_{i-1, j}\right)^{2}} \\
L_{i, j}=2\left[\frac{M U_{i, j-1}}{\Delta h_{1}\left(\Delta h_{1}+\Delta h_{3}\right)}+\frac{M U_{i+1, j}}{\Delta h_{2}\left(\Delta h_{2}+\Delta h_{4}\right)}+\right. \\
\left.+\frac{M U_{i-1, j}}{\Delta h_{4}\left(\Delta h_{2}+\Delta h_{4}\right)}-M U_{i, j}\left(\frac{1}{\Delta h_{1} \cdot \Delta h_{3}}+\frac{1}{\Delta h_{2} \cdot \Delta h_{4}}\right)\right]
\end{gathered}
$$

\section{References Cited}

Press, W.H., Teukolsky, S.A., Vetterling, W.T., and Flannery, B.P., 1992, Numerical recipes in Fortran 77-The Art of Scientific Computing (2nd ed.), v. 1: Cambridge University Press, New York, N.Y., p. 921.

Zwillinger, Daniel, 1997, Handbook of differential equations (3d ed.): Academic Press, p. 801. 
Publishing support provided by the U.S. Geological Survey Publishing Network, Tacoma Publishing Service Center

For additional information contact:

Director, Menlo Park Science Center

U.S. Geological Survey

345 Middlefield Road MS 977

Menlo Park, California 94025

http://earthquake.usgs.gov/regional/nca/ 


\section{趈}

웅 